EDUARDO ALVES DA COSTA

\title{
CONTROLE DA SUSPENSÃO ELETROMAGNÉTICA DE UM VEÍCULO MAGLEV
}

\author{
Dissertação apresentada à Escola \\ Politécnica da Universidade de São Paulo \\ para obtenção do Título de Mestre em \\ Engenharia.
}

São Paulo 
EDUARDO ALVES DA COSTA

\title{
CONTROLE DA SUSPENSÃO ELETROMAGNÉTICA DE UM VEÍCULO MAGLEV
}

\author{
Dissertação apresentada à Escola \\ Politécnica da Universidade de São Paulo \\ para obtenção do Título de Mestre em \\ Engenharia
}

Área de Concentração:

Engenharia de Sistemas

Orientador:

Prof. Dr. José Jaime da Cruz

São Paulo 
FICHA CATALOGRÁFICA

Costa, Eduardo Alves da

Controle da suspensão eletromagnética de um veículo MAGLEV I Eduardo Alves da Costa - São Paulo, 2004. $113 \mathrm{p}$.

Dissertação (Mestrado) - Escola Politécnica da Universidade de São Paulo. Departamento de Engenharia de Telecomunicações e Controle.

1. Controle (Teoria e sistemas de controle) 2. Modelagem matemática I. Universidade de São Paulo. Escola Politécnica. Departamento de Engenharia de Telecomunicações e Controle II. $t$ 
À minha família 


\section{AGRADECIMENTOS}

Ao grande professor e orientador Dr. José Jaime da Cruz, pelo apoio, incentivo e por ajudar-me a encontrar os caminhos que levaram à conclusão deste trabalho.

À minha esposa Ana Rosa e ao meu filho Vinícius, pelo incentivo, compreensão e paciência durante as longas horas utilizadas na execução deste sonho.

Aos meus pais Benito e Maura, e minha irmã Janet que com entusiasmo me incentivaram.

Aos professores Dr.Alexandre e Dr.Anselmo, pelo apoio.

Ao CNPq - Conselho Nacional de Desenvolvimento Científico e Tecnológico, pelo apoio financeiro na forma de bolsa, durante parte desta pesquisa.

Ao Centro Federal de Educação Tecnológica de São Paulo (CEFET-SP), que me possibilitou maior dedicação neste trabalho.

A todos que, direta ou indiretamente, contribuíram com este trabalho. 


\section{RESUMO}

O controle e otimização da operação da suspensão eletromagnética de um protótipo de veículo MAGLEV são apresentados neste trabalho. Este tipo de sistema é inerentemente instável e altamente não linear, sendo um excelente exemplo para o estudo e comparação de diferentes metodologias de controle. Nos últimos anos, experiências significativas com veículos levitados têm ganhado força. A arquitetura do veículo consiste de quatro atuadores eletromagnéticos, quatro sensores de entreferros e quatro acelerômetros, todos instalados nos cantos do veículo. A dinâmica do sistema é descrita através de três modelos matemáticos diferentes: modelo de corpo rígido com três graus de liberdade (movimentos vertical, de rolagem e de arfagem), modelo de corpo não rígido, onde está presente mais um grau de liberdade (movimento de torção), e modelo SISO com quatro movimentos verticais independentes. Blocos de compatibilização de variáveis são utilizados devido à diferença no número de variáveis do modelo matemático com o número de variáveis medidas e de atuação disponíveis no veículo. Os acelerômetros, usados para estimar as velocidades do veículo, foram instalados com o objetivo de estudarse o controle ótimo da suspensão por realimentação de estados com o emprego da técnica do Regulador Linear Quadrático (RLQ) e utilização do método do lugar das raízes para definição dos pólos de malha fechada. Para efeito de comparação foi implementado um controle $\mathrm{H}_{2} / \mathrm{H}_{\infty}$ por realimentação da saída. Controladores independentes para cada grau de liberdade foram simulados no software MATLAB e depois implementados e testados no veículo utilizando uma placa de aquisição de dados instalada no microcomputador. A preocupação principal do projeto dos controladores foi a estabilidade do sistema na ocorrência de perturbações que desviem o entreferro de seu valor nominal. Exceto para o sistema com controle RLQ e modelo de corpo rígido, os resultados experimentais obtidos com o protótipo mostram que o sistema em malha fechada é estável e apresenta uma resposta transiente satisfatória. O sistema com controle RLQ e modelo de corpo não rígido apresentou a melhor performance entre todas as alternativas testadas. 


\begin{abstract}
The control and optimization of the electromagnetic suspension operation of a MAGLEV vehicle prototype are presented in this work. This kind of system is inherently unstable and highly nonlinear, being an excellent example for the study and comparison of different control methodologies. In the last years significant experience has been gained with levitated vehicles. The vehicle architecture consists of four electromagnetic actuators, four air gap sensors and four accelerometers, all located at the corners of the vehicle. The vehicle dynamics is described through three different mathematical models: rigid body model, with three degrees of freedom (heave, roll and pitch motions), not-rigid body model, with an additional degree of freedom (torsion motion) and SISO model, with four independent heave motions. Blocks to make variables compatible are used in based on the difference between the number of variables of the mathematical model and the number of measured and actuation variables available in the vehicle. The accelerometers, used to estimate the speeds of the vehicle, were installed in order to study the optimal control of the suspension for states feedback. The Linear Quadratic Regulator (LQR) technique combined with the root locus method for definition of the closed-loop poles were used. The $\mathrm{H}_{2} / \mathrm{H}_{\infty}$ controller with output feedback was implemented for sake of comparison. Independent controllers for each degree of freedom were simulated with MATLAB then implemented and tested in the vehicle using AD/DA converter installed in the microcomputer. The first goal of the controllers' design was the system stability when subject to disturbances that drive the air away from its nominal value. Except for the system with RLQ controller and rigid body model, the experimental results obtained with the prototype show that the closed-loop system is stable and exhibits a satisfactory transient response. The system with the RLQ controller and not-rigid body model exhibits the best performance among all the alternatives tested.
\end{abstract}




\section{SUMÁRIO}

LISTA DE FIGURAS .............................................................................................I

LISTA DE TABELAS ................................................................................................ III

LISTA DE ABREVIATURAS ....................................................................................IV

LISTA DE SÍMBOLOS................................................................................................. V

1 INTRODUÇÃ $O$......................................................................................................... 1

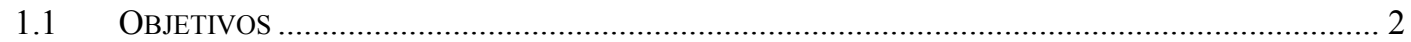

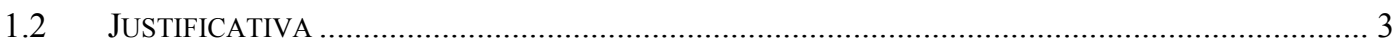

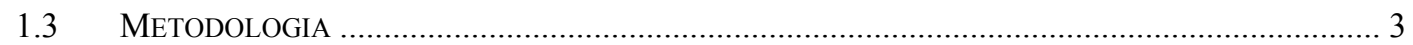

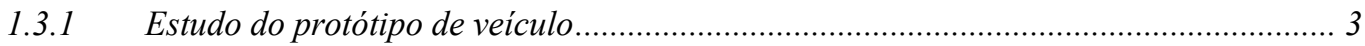

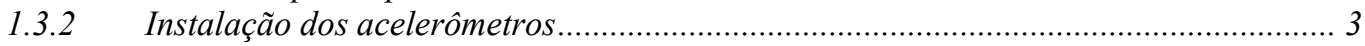

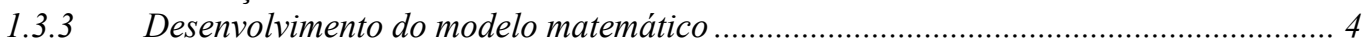

1.3.4 Projeto dos controladores e comparação dos resultados ......................................... 4

1.4 DESCRIÇÃO DO SISTEMA .................................................................................. 5

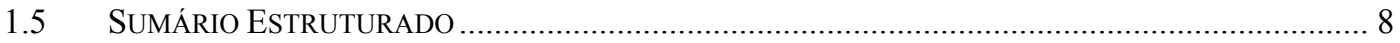

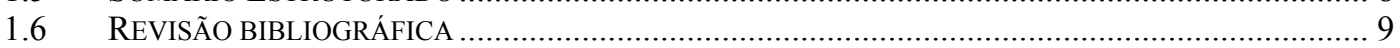

2 SENSOR DE ACELERAÇÃO ........................................................................ 12

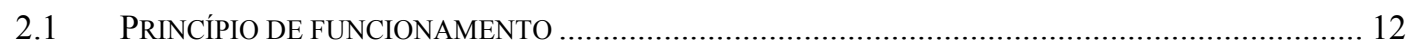

2.2 DESCRIÇÃO GERAL E CARACTERÍSTICAS TÉCNICAS .................................................... 15

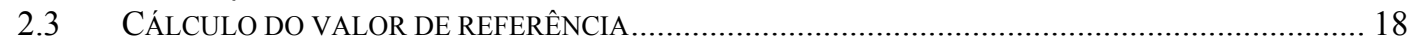

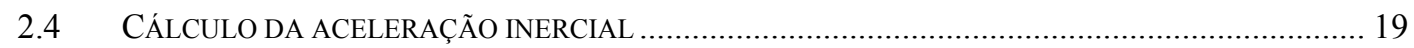

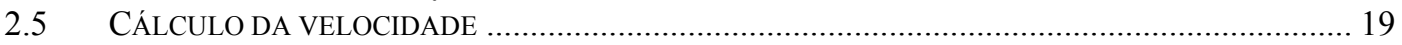

3 MODELO MATEMÁTICO DO SISTEMA E COMPATIBILIZAÇÃO.... 23

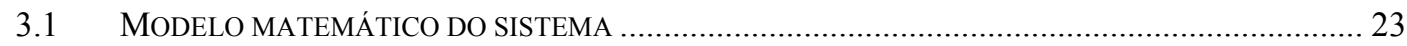

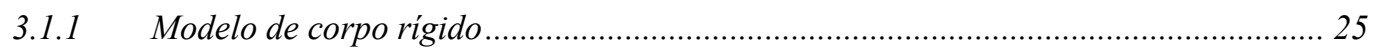

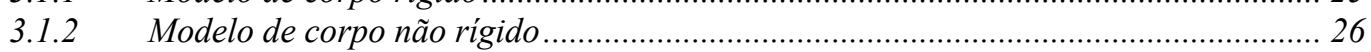

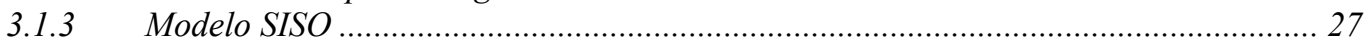

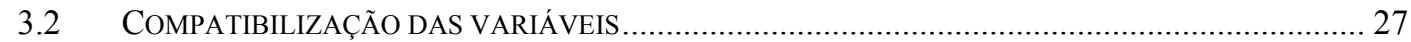

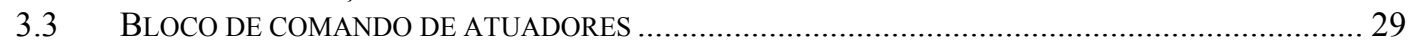

3.3.1 Relação da força eletromagnética com tensão elétrica................................................... 29

3.3.2 Otimização das forças de suspensão para o modelo de corpo rígido ........................... 30

3.3.3 Distribuição das forças para o modelo de corpo não rigido ........................................ 36

3.3.4 Distribuição das forças para o modelo SISO........................................................... 37

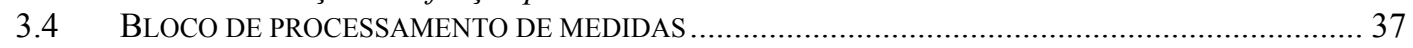

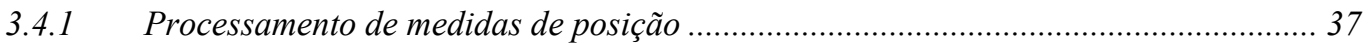

3.4.2 Processamento de medidas de velocidade.............................................................. 41

\section{RLQ COM ALOCAÇÃO DOS PÓLOS EM MALHA FECHADA VIA}

\section{LUGAR DAS RAÍZES .............................................................................................. 42}

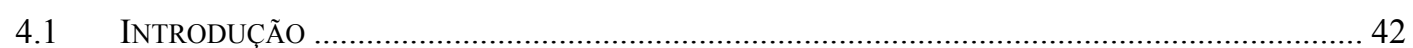

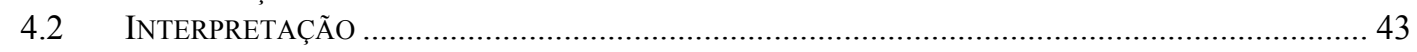

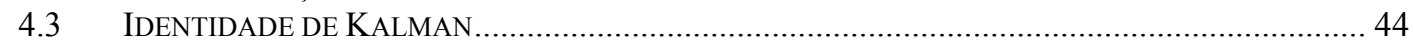

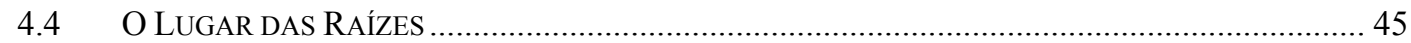

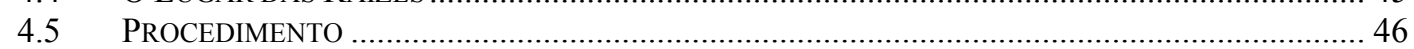


5 PROJETO DOS CONTROLADORES ........................................................... 48

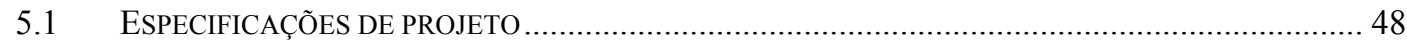

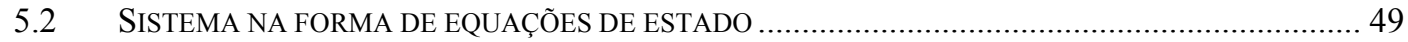

5.2.1 Movimento vertical com controlador ....................................................................... 49

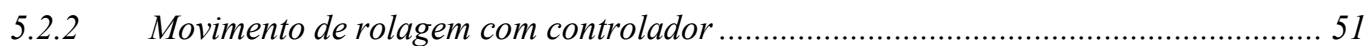

5.2.3 Movimento de arfagem com controlador ............................................................. 52

5.2.4 Movimento de torção com controlador ............................................................ 54

5.3 ANÁLISE DO SISTEMA EM REGIME ESTACIONÁRIO ........................................................... 56

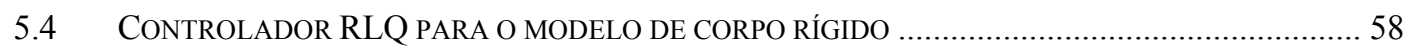

5.4.1 Controlador do movimento vertical ................................................................... 59

5.4.2 Controlador do movimento de rolagem.............................................................. 62

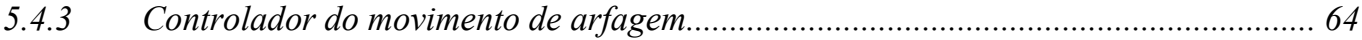

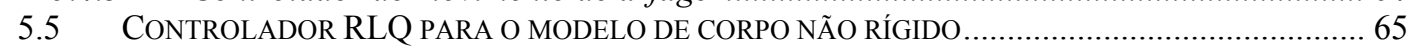

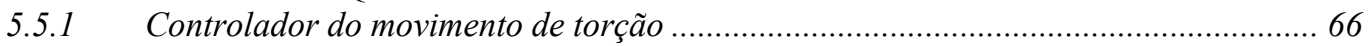

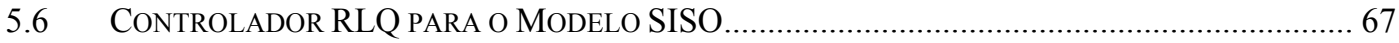

5.7 CONTROLADOR $\mathrm{H}_{2} / \mathrm{H}_{\infty}$ PARA O MODELO COM REALIMENTAÇÃO DA SAÍDA ........................... 68

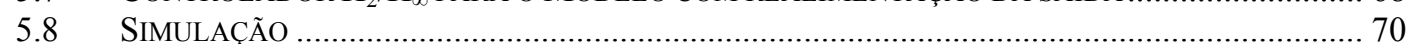

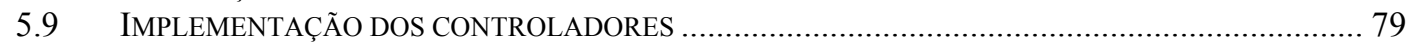

6 RESULTADOS E ANÁLISE COMPARATIVA................................................. 80

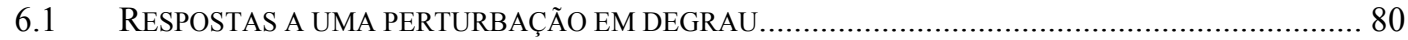

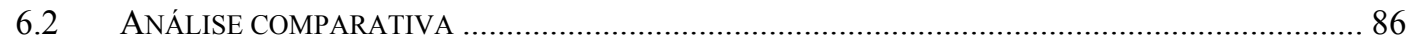

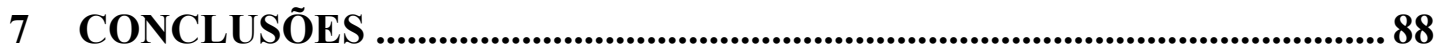

ANEXO A - CIRCUITO REGULADOR DE VOLTAGEM................................91

ANEXO B - CALIBRAÇÃO DOS SENSORES DE POSIÇÃO ..........................92

ANEXO C - CALIBRAÇÃO DOS ATUADORES..............................................94

ANEXO D - PROGRAMA DE CONTROLE .................................................99

REFERÊNCIAS BIBLIOGRÁFICAS ............................................................... 111 


\section{LISTA DE FIGURAS}

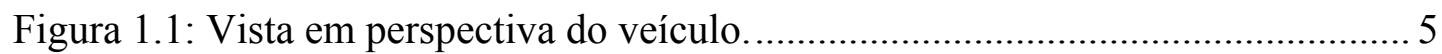

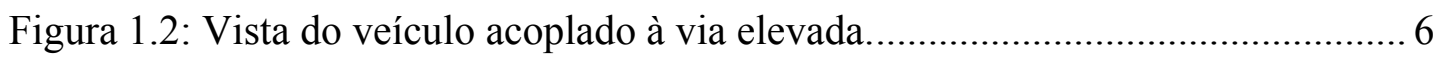

Figura 2.1: Esquema do sistema massa-mola-amortecedor. ................................... 13

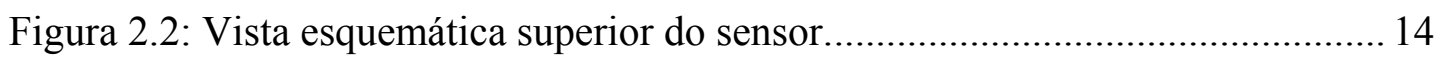

Figura 2.3: Diagrama do sensor em repouso.......................................................... 15

Figura 2.4: Resposta do sensor a uma aceleração externa. ....................................... 15

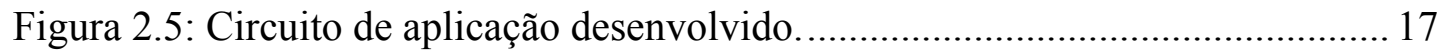

Figura 2.6: Diagrama de blocos em s (Laplace) para cálculo da velocidade............ 19

Figura 2.7: Diagrama de blocos em Z (discreto) para cálculo da velocidade........... 20

Figura 2.8: Aceleração com componente DC e velocidade com e sem filtro............ 21

Figura 2.9: Velocidade com integrador e filtro passa-altas de $w_{c}=1 \mathrm{rad} / \mathrm{s}$ e $10 \mathrm{rad} / \mathrm{s}$.

Figura 3.1: Esquema de forças de suspensão......................................................... 23

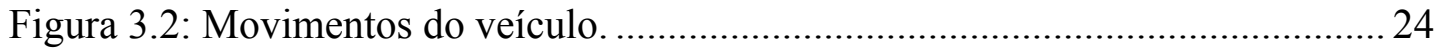

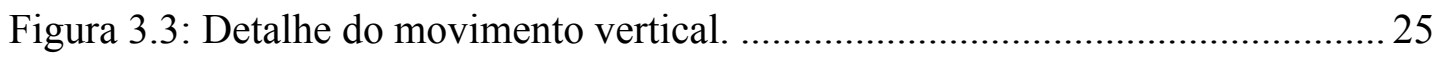

Figura 3.4: Blocos de compatibilização das variáveis do modelo de corpo rígido.... 28

Figura 3.5: Blocos de compatibilização das variáveis do modelo de corpo não rígido.

Figura 3.6: Blocos de compatibilização das variáveis do modelo SISO.................... 29

Figura 3.7: Detalhamento do modelo de corpo rígido com realimentação da saída. . 29

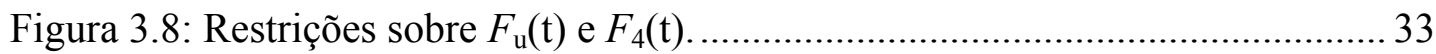

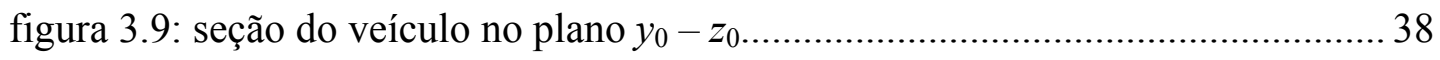

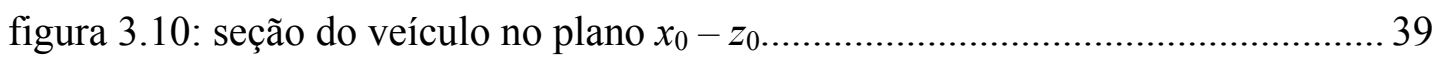

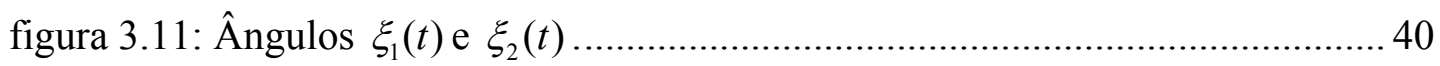

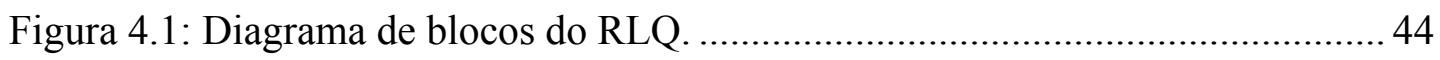

Figura 4.2: Diagrama de blocos para o Lugar das Raízes........................................ 46

Figura 5.1: Diagrama de blocos do movimento vertical com o controlador.............. 49

Figura 5.2: Diagrama de blocos do movimento de rolagem com o controlador........ 51

Figura 5.3: Diagrama de blocos do movimento de arfagem com o controlador.........53 
Figura 5.4: Diagrama de blocos do movimento de torção com o controlador. 54

Figura 5.5: Sistema em malha fechada - modelo de corpo rígido............................ 59

Figura 5.6: Lugar das raízes para projeto do controle vertical.................................. 61

Figura 5.7: Sistema em malha fechada - modelo de corpo não rígido. ..................... 65

Figura 5.8: Sistema em malha fechada - modelo SISO …...................................... 67

Figura 5.9: Sistema em malha fechada - modelo de corpo rígido com realimentação

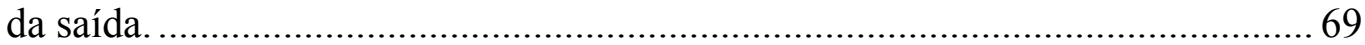

Figura 5.10: Diagrama de blocos para simulação do controle vertical...................... 70

Figura 5.10: Diagrama de blocos para simulação do controle de rolagem. ............... 71

Figura 5.12: Diagrama de blocos para simulação do controle de arfagem................ 71

Figura 5.13: Diagrama de blocos para simulação das tensões $v_{i}(t)$ do atuador......... 72

Figura 5.14: Resposta ao degrau e perturbação para o controle vertical. ................... 72

Figura 5.15: Tensões nos atuadores para o controle vertical.................................. 73

Figura 5.16: Resposta ao degrau e perturbação para o controle de rolagem............... 74

Figura 5.17: Tensões nos atuadores para o controle de rolagem. ……..................... 75

Figura 5.18: Resposta ao degrau e perturbação para o controle de arfagem............... 75

Figura 5.19: Tensões nos atuadores para o controle de arfagem. ….......................... 76

Figura 5.20: Resposta ao degrau e perturbação para o controle de torção.................. 77

Figura 5.21: Tensões nos atuadores para o controle de torção. ................................. 77

Figura 5.22: Resposta ao degrau e perturbação para o controle SISO....................... 78

Figura 5.23: Tensão $v_{1}(\mathrm{t})$ no atuador para o controle SISO ..................................... 79

Figura 6.1: Resposta à perturbação em degrau com controle RLQ e modelo de corpo rígido. 81

Figura 6.2: Resposta à perturbação em degrau com controle RLQ e modelo de corpo não rígido. 82

Figura 6.3: Resposta à perturbação em degrau com controle RLQ e modelo SISO.. 83 Figura 6.4: Resposta à perturbação em degrau com controle $\mathrm{H}_{2} / \mathrm{H}_{\infty}$ e modelo de

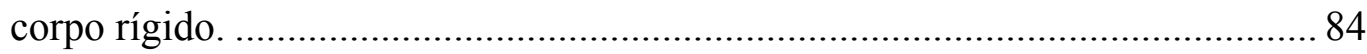

Figura A.1: Circuito regulador de voltagem. 


\section{LISTA DE TABELAS}

Tabela 1.1: Dados do veículo............................................................................. 7

Tabela 2.1: Dados técnicos do acelerômetro $\left(T_{\mathrm{A}}=25^{\circ} \mathrm{C}, V_{\mathrm{DD}}=5 \mathrm{~V}\right.$ e acel. $\left.=0 g\right) \ldots \ldots \ldots . .15$

Tabela 2.2: Sensibilidade real dos acelerômetros.................................................. 17

Tabela 6.1: Valores R.M.S. para as respostas à perturbação em degrau.................... 87

Tabela B.1.: Dados dos entreferros em Volts e milímetros. ................................... 93

Tabela C.1.: Dados da relação força aplicada por tensão em cada atuador. ............. 95

Tabela C.2.: Valores de entreferro em Volts e milímetros. ..................................... 96

Tabela C.3.: Parâmetros para cálculo de $k_{\mathrm{vi}}$............................................................ 97

Tabela C.4.: Constantes $k_{\mathrm{vi}}$ para os eletroímãs....................................................... 98 


\section{LISTA DE ABREVIATURAS}

$\begin{array}{ll}\text { EAR } & \text { Equação Algébrica de Riccati } \\ \text { EDS } & \text { "ElectroDynamic Suspension" } \\ \text { EMS } & \text { "ElectroMagnetic Suspension" } \\ \text { MAGLEV } & \text { "Magnetic Levitation" } \\ \text { MEMS } & \text { "MicroElectroMechanical Systems" } \\ \text { RLQ } & \text { Regulador Linear Quadrático } \\ \text { R.M.S. } & \text { "Root Mean Square" } \\ \text { SISO } & \text { "Single Input Single Output } \\ \text { USP } & \text { Universidade de São Paulo }\end{array}$




\section{LISTA DE SÍMBOLOS}

$\begin{array}{ll}F_{a} & \text { força provocada pela aceleração. } \\ m_{a} & \text { massa conhecida. } \\ a_{a} & \text { aceleração. } \\ b_{a} & \text { coeficiente de viscosidade. } \\ k_{a} & \text { constante da mola. } \\ z_{a} & \text { deslocamento da massa do acelerômetro. } \\ g & \text { aceleração da gravidade. } \\ T_{\mathrm{A}} & \text { temperatura de operação do acelerômetro. } \\ V_{\mathrm{DD}} & \text { tensão de alimentação do acelerômetro. } \\ V_{\mathrm{S}} & \text { tensão de saída do regulador de voltagem. } \\ U_{+\mathrm{g}} & \text { tensão na saída do acelerômetro para }+1 g . \\ U_{-\mathrm{g}} & \text { tensão na saída do acelerômetro para }-1 g . \\ V_{\mathrm{MID}} & \text { tensão de referência para o amplificador operacional. } \\ \mathrm{UCA} & \text { tensão de saída do acelerômetro. } \\ x_{\mathrm{i}} & \text { valores lidos. } \\ \bar{x} & \text { média aritmética dos valores lidos. } \\ n & \text { número de valores lidos. } \\ \ddot{z}_{p} & \text { tensão para aceleração inercial nula. } \\ \ddot{z}_{p p}(t) & \text { aceleração inercial medida no eixo } z . \\ F r e q & \text { aceleração inercial no eixo } z . \\ & \text { freqüência de amostragem. } \\ & \end{array}$




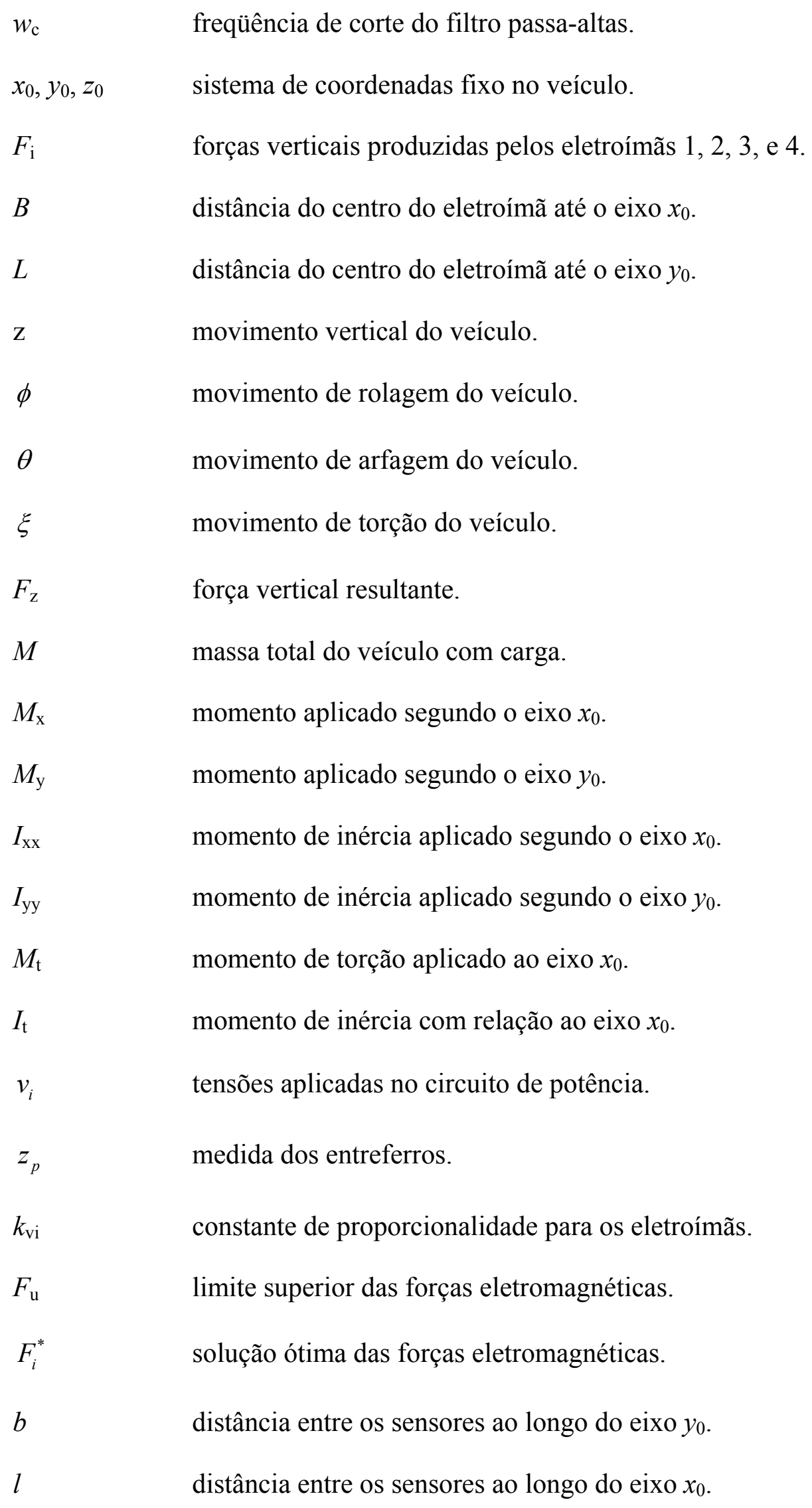




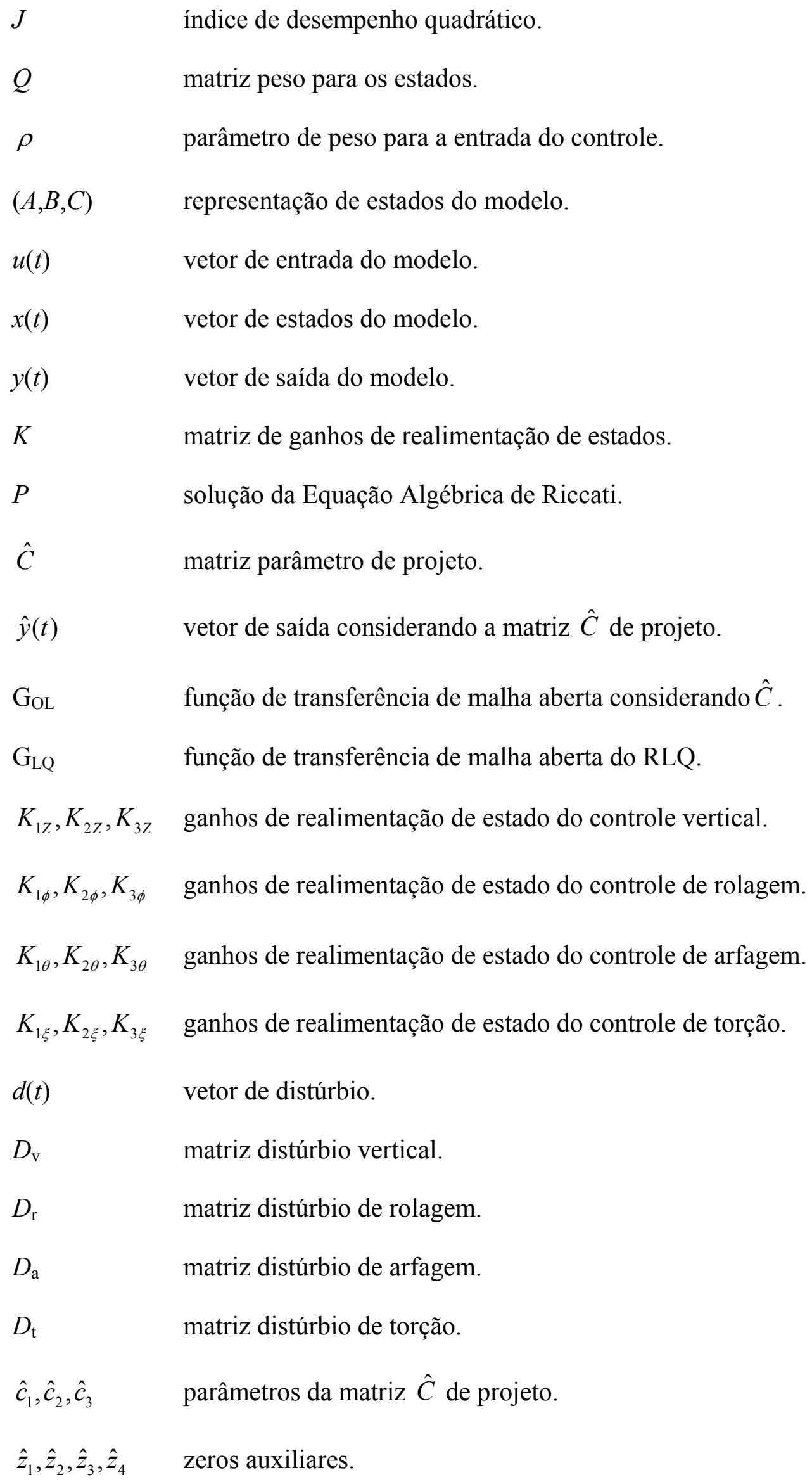


$p_{1}, p_{2}, p_{3} \quad$ pólos de malha fechada do sistema.

$t_{\mathrm{r}}$ tempo de subida.

$t_{\mathrm{s}}$ tempo de acomodação.

$M_{\mathrm{p}}$ sobre-sinal máximo em valor percentual.

$d_{e}$ valor do desvio em relação ao valor em regime estacionário.

$t_{\mathrm{p}}$ tempo de acomodação devido à perturbação.

$z_{\mathrm{mm}}$ entreferro medido em milímetros.

$z_{\mathrm{v}}$ entreferro medido em volts.

$i(t) \quad$ corrente elétrica na bobina.

$\mu_{0} \quad$ permeabilidade do ar.

$a, c \quad$ dimensões do eletroímã.

$N \quad$ número de espiras. 


\section{Capítulo 1}

\section{INTRODUÇÃO}

A levitação magnética tem diversas aplicações onde o atrito pode ser reduzido ou até eliminado, como nos mancais de motores. Importante e promissora aplicação é encontrada em sistemas de transporte baseados em levitação magnética (MAGLEV).

Os sistemas MAGLEV envolvem a levitação, propulsão e guiagem. Este trabalho tem o enfoque na levitação do veículo, onde duas tecnologias são dominantes: sistemas eletrodinâmicos (EDS - "ElectroDynamic Suspension") e sistemas eletromagnéticos (EMS - "ElectroMagnetic Suspension").

A suspensão eletrodinâmica age por meio de forças de repulsão. Quando um material condutor está próximo de uma bobina que produz campo magnético variável ou que esteja em movimento relativo, correntes parasitas são induzidas no condutor e, conseqüentemente, forças de repulsão. Na aplicação em MAGLEV o campo magnético é gerado por bobinas supercondutoras instaladas nos veículos; quando em movimento a variação de fluxo induz corrente em bobinas passivas instaladas nos trilhos e esta corrente interage com o campo magnético produzindo força de repulsão que levita o trem.

As vantagens desta tecnologia estão na estabilidade do sistema em malha aberta e entreferro maior que $80 \mathrm{~mm}$, mas apresenta algumas desvantagens: sistema sub-amortecido, elevado campo magnético no compartimento de passageiros e necessidade de rodas para baixas velocidades $(<100 \mathrm{~km} / \mathrm{h})$, pois o veículo necessita estar acima desta velocidade para levitar.

A tecnologia de suspensão eletrodinâmica tem o seu maior desenvolvimento em uma linha experimental de transporte no Japão, projeto MLX.

Outro projeto utilizando suspensão eletrodinâmica está em desenvolvimento nos Estados Unidos da América, chamado de Inductrack (POST; RYUTOV, 2000). Nesta tecnologia, em vez de bobinas supercondutoras, são utilizados, para excitação, ímãs permanentes agrupados em forma especial de matriz de Halbach, que aumenta o campo magnético abaixo da configuração da matriz e anula o campo acima da 
matriz. A vantagem desta tecnologia está na obtenção de um elevado campo magnético sem a necessidade do uso de bobinas supercondutoras de custo elevado.

A suspensão eletromagnética age por meio de forças de atração que são produzidas por eletroímãs alimentados por corrente contínua. Neste tipo de sistema é inerente a instabilidade em malha aberta, conseqüência do teorema de Earnshaw (EARNSHAW, 1839; VALADARES, 2001). A estabilidade é obtida com a utilização de controlador em malha fechada.

Uma vantagem do sistema de suspensão eletromagnética é que o veículo levita mesmo parado, mas tem a desvantagem do sistema ser instável e não linear, necessitando de um controle robusto e imune à falhas.

A tecnologia por suspensão eletromagnética é encontrada com destaque no projeto Transrapid, desenvolvido na Alemanha e empregado comercialmente em uma primeira linha em Shanghai na China, que entrou em operação em 29 de dezembro de 2003, atingindo uma velocidade recorde em sistemas comerciais $(501 \mathrm{~km} / \mathrm{h})$.

\subsection{Objetivos}

Neste trabalho foram instalados acelerômetros em um protótipo de veículo com levitação eletromagnética (MAGLEV) por atração, com o objetivo de estudar o controle ótimo da suspensão por realimentação de estados com o emprego da técnica do Regulador Linear Quadrático (RLQ) com alocação dos pólos em malha fechada via lugar das raízes. Para efeito de comparação foi implementado um controle $\mathrm{H}_{2} / \mathrm{H}_{\infty}$ por realimentação da saída e independente para cada grau de liberdade.

Os controladores foram implementados considerando a dinâmica do modelo matemático como sendo de corpo rígido, de corpo não rígido e SISO.

Sendo a suspensão por atração eletromagnética altamente não linear e instável em malha aberta, a preocupação principal do projeto dos controladores é a estabilidade do sistema na ocorrência de perturbações que desviem o entreferro de seu valor nominal. 
Visto que no modelo matemático de corpo rígido o sistema tem mais atuadores do que variáveis de controle, foi desenvolvido um procedimento de otimização das forças eletromagnéticas de suspensão, na tentativa de minimizar a máxima força entre todas que agem nos atuadores.

\subsection{Justificativa}

Os sistemas de levitação magnética são utilizados em diversas aplicações, tais como mancais, onde permitem alta velocidade de rotação, eficiência e pouco desgaste mecânico; em veículos de transporte de alta velocidade na sua suspensão, guiagem e propulsão.

O estudo e desenvolvimento de novas técnicas de controle, aplicadas a estes sistemas, justifica-se por ser uma área promissora e com grande potencial de desenvolvimento.

\subsection{Metodologia}

O projeto desenvolvido tem como ponto de partida o protótipo de um veículo de levitação eletromagnética desenvolvido na Escola Politécnica da USP, (BITTAR, 1998). Neste veículo foram feitas implementações para que se explorem novas técnicas de controle.

O projeto foi realizado através das etapas descritas a seguir.

\subsubsection{Estudo do protótipo de veículo}

Nesta etapa estudou-se tanto o hardware como o software do protótipo existente do veículo levitado eletromagneticamente.

\subsubsection{Instalação dos acelerômetros}

Para obter informações dos estados do sistema, foi instalado em cada extremidade do veículo um acelerômetro, modelo ADXL 105, para medir a 
aceleração e indiretamente a velocidade dos movimentos vertical, de rolagem, de arfagem e torção.

Inicialmente foi feito um estudo do funcionamento do acelerômetro, onde se verificou o modo de instalação, alimentação e demais características técnicas. Como resultado surgiu a necessidade de instalação de uma fonte de alimentação $5 \mathrm{Vcc}$ regulada.

Após a instalação dos acelerômetros, devido ao elevado nível de ruído no sinal de saída, foi utilizado entre o sensor e a placa de aquisição de dados um cabo de par trançado com blindagem individual, na tentativa de minimizar o nível de ruído do sistema.

\subsubsection{Desenvolvimento do modelo matemático}

Para o sistema são considerados três modelos matemáticos distintos. O modelo de corpo rígido considera os movimentos: vertical ("heave"), de rolagem ("roll") e o de arfagem ("pitch"). O modelo de corpo não rígido acrescenta o movimento de torção aos movimentos do corpo rígido. No modelo SISO são considerados, para cada canto do veículo, quatro movimentos verticais independentes.

\subsubsection{Projeto dos controladores e comparação dos resultados}

Neste projeto foi utilizada a técnica de controle RLQ com alocação dos pólos em malha fechada via lugar das raízes. Estes controles ótimos foram implementados via realimentação de estados gerados pelos acelerômetros e pelos sensores de entreferro.

Como complemento desenvolveu-se um procedimento de otimização das forças eletromagnéticas de suspensão.

Os controles foram simulados no software MATLAB e depois implementados e testados no veículo utilizando uma placa de aquisição de dados instalada no microcomputador.

Toda a rotina do controlador foi desenvolvida em linguagem Pascal de programação. 
Por último foi realizada uma análise comparativa dos resultados obtidos entre os diversos controladores, considerando as respostas às perturbações.

\subsection{Descrição do sistema}

O sistema idealizado consiste na adaptação de um protótipo de um veículo existente, (BITTAR, 1998), que é levitado eletromagneticamente. Foram instalados quatro novos acelerômetros que medem parte das variáveis de estado (aceleração e indiretamente a velocidade) do veículo.

Nas figuras 1.1 e 1.2 são apresentados, respectivamente, detalhes dimensionais do veículo e uma vista frontal do veículo acoplado à via elevada.

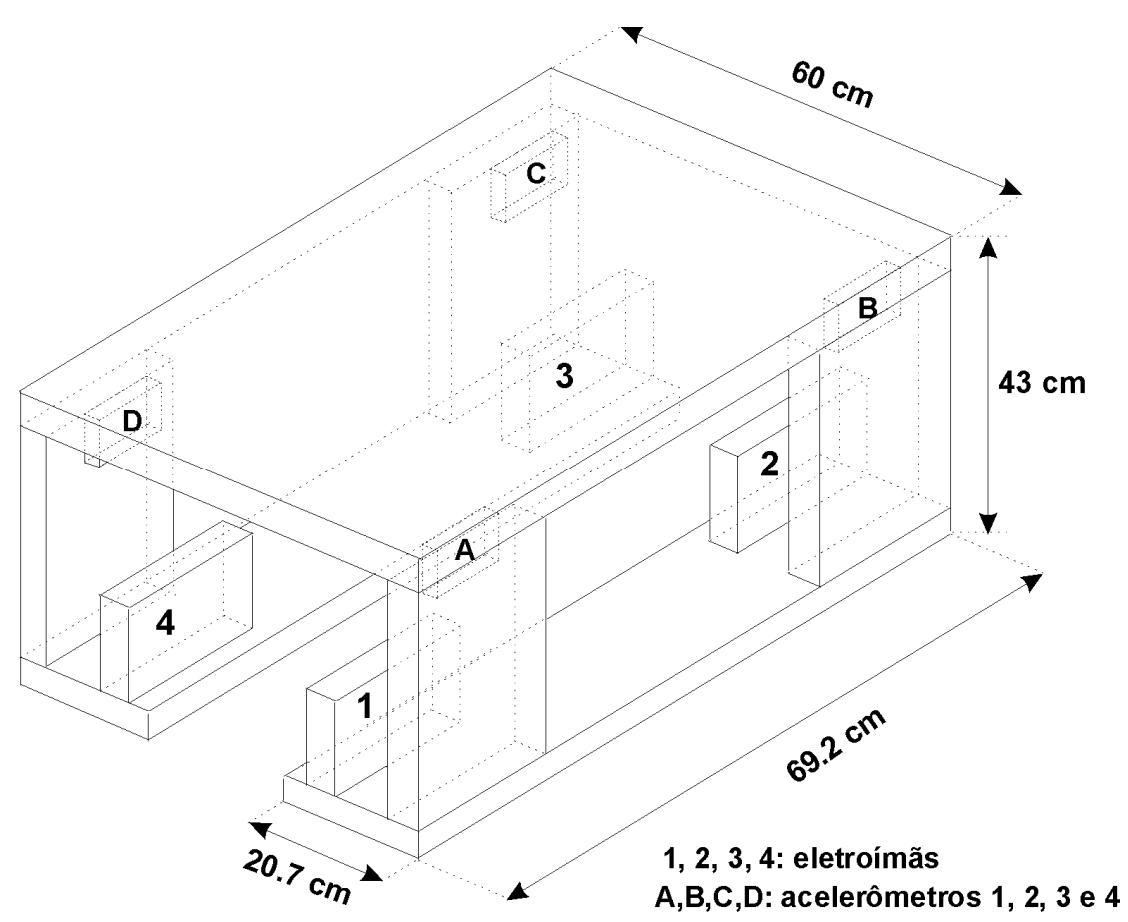

Figura 1.1: Vista em perspectiva do veículo.

(Fonte: BITTAR, 1998). 


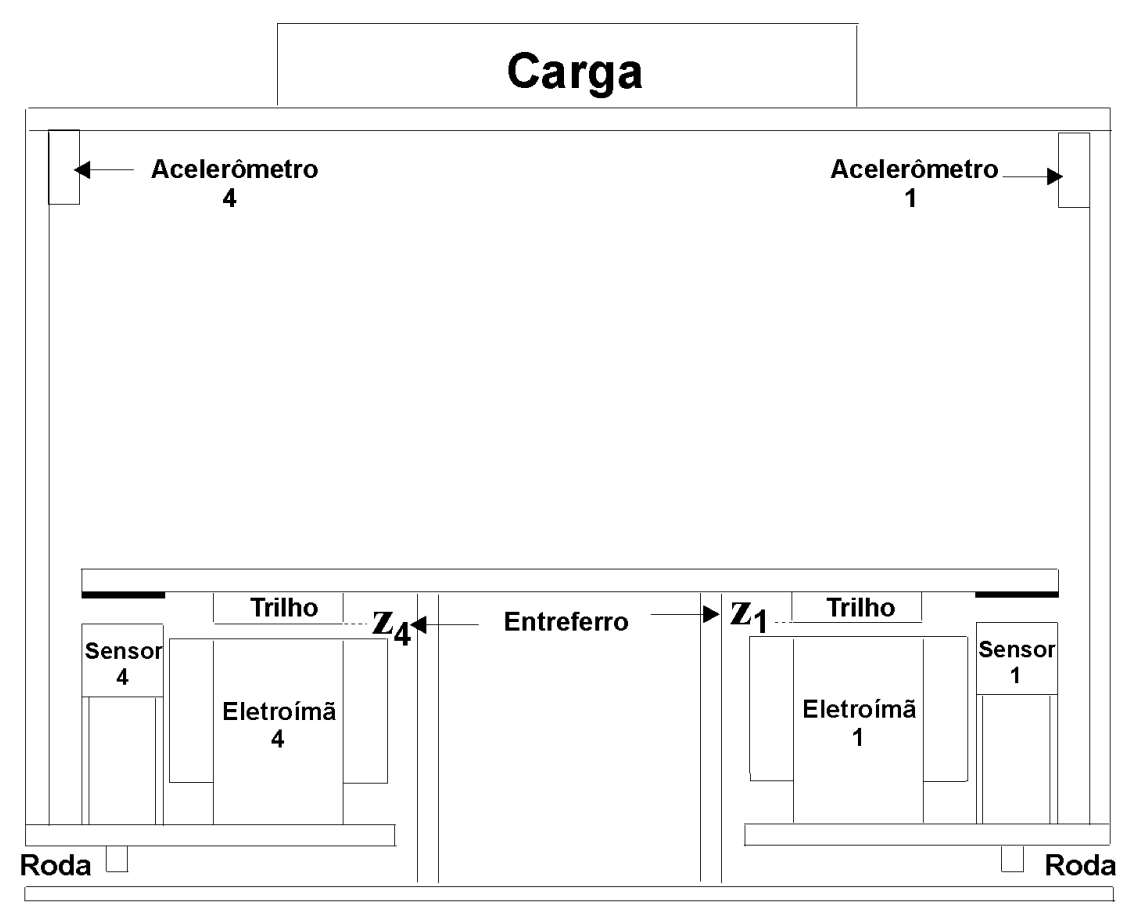

Figura 1.2: Vista do veículo acoplado à via elevada.

(Fonte: BITTAR, 1998).

O veículo possui quatro eletroímãs, quatro sensores de proximidade indutivos e quatro acelerômetros localizados nas extremidades do protótipo. A levitação é realizada através de forças de atração produzidas pelos eletroímãs.

Os sensores de proximidade servem para medir os entreferros dos eletroímãs, que são os atuadores do sistema. Estes sensores fornecem uma tensão contínua proporcional à distância medida.

Na tentativa de tornar o veículo mais rígido, foram instaladas em cada canto do veículo, na parte superior, barras transversais para fixação da parte superior com a lateral. 
A tabela 1.1 apresenta os principais dados do protótipo.

Tabela 1.1: Dados do veículo.

(Fonte: BITTAR, 1998)

\begin{tabular}{|l|l|}
\hline Comprimento & $69,2 \mathrm{~cm}$ \\
\hline Largura & $60 \mathrm{~cm}$ \\
\hline Altura & $43 \mathrm{~cm}$ \\
\hline Massa* & $88,3 \mathrm{~kg}$ \\
\hline Carga & $9,3 \mathrm{~kg}$ \\
\hline Entreferro em regime estacionário & $5 \mathrm{~mm}$ \\
\hline Tensão de alimentação & $55 \mathrm{Vcc}$ \\
\hline Corrente em cada bobina em regime estacionário & $1,7 \mathrm{~A}$ \\
\hline Potência total em levitação & $374 \mathrm{~W}$ \\
\hline
\end{tabular}

* considerando acelerômetros e barras transversais

O veículo e a via elevada foram construídos em alumínio e os trilhos, com barras de ferro para fechamento do fluxo magnético dos eletroímãs.

Complementando o sistema, tem-se um circuito de potência que foi projetado para atuar como interface entre a saída do controlador digital e a entrada do sistema (tensão elétrica nas bobinas dos eletroímãs).

O controle digital é realizado pelo microcomputador através das seguintes etapas: leitura dos quatro sensores de entreferro e dos quatro acelerômetros; estimativa dos valores de posição e velocidade, ou variáveis de estado do modelo através de um bloco de "Processamento de Medidas"; a estes valores é aplicado um algoritmo de controle que determina as variáveis de entrada do modelo; em seguida no bloco de "Comando de Atuadores" obtêm-se as forças eletromagnéticas de suspensão e indiretamente a tensão aplicada na entrada do circuito de potência; o circuito de potência por sua vez fornece a corrente ao eletroímã para que o protótipo levite. 


\subsection{Sumário Estruturado}

Este trabalho envolve a implementação de acelerômetros e de novos controladores para o sistema de suspensão eletromagnética de um protótipo de veículo.

Neste capítulo 1 são apresentados os objetivos, as justificativas, a descrição do sistema e a metodologia empregada neste projeto.

Os acelerômetros utilizados na medição das variáveis de estado são apresentados no capítulo 2. Neste está descrito o sensor, seu funcionamento, circuito de alimentação e tratamento do sinal no microcomputador, para obtenção da medida de velocidade.

No capítulo 3 estão descritos os três modelos matemáticos do sistema. O modelo de corpo rígido considera os movimentos: vertical, de rolagem e o de arfagem. O modelo de corpo não rígido acrescenta o movimento de torção aos movimentos do corpo rígido. No modelo SISO são considerados para cada canto do veículo quatro movimentos verticais de mesma natureza. Ainda neste capítulo, são apresentados os blocos de compatibilização de variáveis, devido à diferença no número de variáveis do modelo matemático com o número de variáveis disponíveis no veículo. Estes blocos são "Processamento de Medidas" e "Comando de Atuadores".

No capítulo 4 tem-se um resumo da teoria e do procedimento utilizados no projeto dos controladores do tipo RLQ. Neste as matrizes de peso são escolhidas alocando-se os pólos de malha fechada através dos lugares das raízes.

No capítulo 5 está descrito o projeto e a simulação dos controladores considerando a dinâmica do modelo matemático como sendo de corpo rígido, de corpo não rígido e SISO. Foram desenvolvidos controladores independentes, do tipo RLQ, para cada um dos graus de liberdade: movimento vertical, de rolagem, de arfagem e de torção. Para o modelo de corpo rígido também está descrito o controlador $\mathrm{H}_{2} / \mathrm{H}_{\infty}$ por realimentação da saída e independente para cada grau de liberdade. 
Os resultados e análise comparativa fazem parte do capítulo 6 Com a aplicação de uma perturbação em degrau na planta, são feitas comparações entre as diversas técnicas de controle empregadas neste projeto.

As considerações e conclusões do trabalho com relação aos resultados obtidos neste projeto estão descritas no capítulo 7.

Finalmente, nos anexos estão apresentadas informações que complementam e esclarecem este projeto. No anexo A tem-se o circuito do regulador de voltagem que alimenta os acelerômetros. Nos anexos B e C estão apresentados, respectivamente, a calibração dos sensores de posição e calibração dos atuadores. No anexo D tem-se o programa desenvolvido em Pascal para controle da levitação do protótipo considerando o modelo de corpo não rígido.

\subsection{Revisão bibliográfica}

Nesta seção, a bibliografia que foi utilizada no desenvolvimento da dissertação, está apresentada com breve descrição do conteúdo ou aspectos relevantes de cada uma das obras pesquisadas.

Para conhecimento do estágio atual dos diversos sistemas MAGLEV utilizados em transporte, têm-se nos trabalhos publicados por (CASSAT; JUGER, 2002; VUCHIC; CASELLO, 2002) uma apresentação dos aspectos tecnológicos e escolhas de projetos MAGLEV ao redor do mundo: Alemanha com o Transrapid, Japão com o MLX, Estados Unidos com o Inductrack e Suíça com o Swissmetro. São investigados e comparados a propulsão, levitação e guiagem do veículo.

A nova tecnologia Inductrack para levitação magnética e o conceito de matriz de Halbach para configuração de ímãs permanentes estão disponíveis em (POST; RYUTOV, 2000).

O trabalho de (SINHA, 1987) é um dos poucos livros que aborda de maneira abrangente o estudo de suspensão eletromagnética. Este livro trata de supercondutividade, sistema de levitação eletromagnética e eletrodinâmica, estabilidade da suspensão eletromagnética, utilização de acelerômetros na realimentação de estados, além de auxiliar na definição do modelamento do sistema 
proposto. Devido à época em que foi escrito está um pouco desatualizado com relação à referência dos projetos em desenvolvimento.

Uma das referências mestras no desenvolvimento deste projeto foi o trabalho de (BITTAR, 1998). Neste é feito um estudo das diversas técnicas de controle aplicadas na suspensão eletromagnética de um protótipo de veículo. São definidos os eletroímãs, sensores de posição e circuito de potência.

Os modelos matemáticos desenvolvidos estão baseados nas referências (SINHA, 1987; BITTAR, 1998).

O trabalho apresentado em (ATHANS, 1981) permitiu o projeto dos controladores utilizando-se da técnica de controle RLQ com alocação dos pólos de malha fechada via lugar das raízes.

As bibliografias (KAILATH, 1980; ORSINI, 1985; OGATA, 1998; LEVINE, 1996) são básicas e importantes no desenvolvimento da técnica de controle RLQ. Outras referências, para controle robusto, são (CRUZ, 1996) com a técnica LQG/LTR e (ZHOU, 1996) com a técnica $\mathrm{H}_{2} / \mathrm{H}_{\infty}$.

O trabalho de (BITTAR, 1993) apresenta projeto de controladores para levitação eletromagnética de uma barra flexível, além do modelamento do sistema, definição do eletroímã e sensor de posição.

O protótipo, modelo e controle de um sistema de levitação e propulsão eletrodinâmica estão apresentados no trabalho de (CAMPO, 2001).

Em (ORSINI, 1984; FALCONE, 1985) são encontrados fundamentos para entendimento da força magnética do eletroímã em função da corrente na bobina, entreferro, dimensão e número de espiras.

Em (GAVIN; MORALES; REILLY, 1998) e (GAVIN, 2001) são encontrados fundamentos para solução do problema de deriva apresentado quando da integração de sinais, neste caso a integração do sinal de aceleração para obtenção de velocidade.

O manual (LYNX, 1997) descreve o modo de funcionamento e programação da placa de leitura e escrita de dados utilizada no projeto. 
Para a montagem e alimentação dos acelerômetros e informações dos sensores de posição, foram utilizadas as bibliografias (ANALOG DEVICE, 1999a; ANALOG DEVICE, 1999b) e as informações disponíveis nas páginas WEB dos fabricantes: National Semiconductor, Analog Devices e Sense - Sensores e Instrumentos. 


\section{Capítulo 2}

\section{SENSOR DE ACELERAÇÃO}

Sensores são implementados no sistema para medir variáveis controladas e devem atender aspectos técnicos relacionados à precisão, largura de banda, ruído e outras características.

Os sensores empregados no protótipo são os de posição, descritos em (BITTAR,1998), e os de aceleração, que medem indiretamente a velocidade vertical do veículo. Neste capítulo estão apresentados o princípio de funcionamento, os circuitos auxiliares, as características técnicas e os detalhes de projeto dos acelerômetros.

Faz parte também deste capítulo o cálculo da velocidade vertical do protótipo.

\subsection{Princípio de funcionamento}

Os acelerômetros utilizam o princípio da Lei de Newton,

$$
F_{a}=m_{a} a_{a}
$$

medindo-se a força $F_{a}$ provocada pela aceleração $a_{a}$ em um objeto de massa $m_{a}$ conhecida. Existem diversas maneiras de medir a força exercida devido à aceleração sobre uma massa conhecida, massa padrão, mas o método mais comum usado em acelerômetros é medir o deslocamento da massa padrão, suspensa por molas. O sistema massa-mola-amortecedor está apresentado na figura 2.1.

Quando o sistema está em equilíbrio tem-se, das forças atuantes na massa padrão, que:

$$
F_{a}=m_{a} a_{a}=b_{a} v_{a}+k_{a} z_{a}
$$


onde $b_{a}$ é o coeficiente de viscosidade, $k_{a}$ a constante da mola, $a_{a}=\frac{d^{2} z_{a}}{d t^{2}} \mathrm{e}$ $v_{a}=\frac{d z_{a}}{d t}$.

Longe da freqüência de ressonância do sistema, o efeito do amortecimento pode ser ignorado, então a aceleração é dada por:

$$
a_{a}=\frac{k_{a}}{m_{a}} z_{a},
$$

ou seja, é função do deslocamento da massa padrão. Um dos métodos utilizados para medir o deslocamento $z_{a}$ é o capacitivo, que mede a variação de capacitância entre placas fixas e móvel.

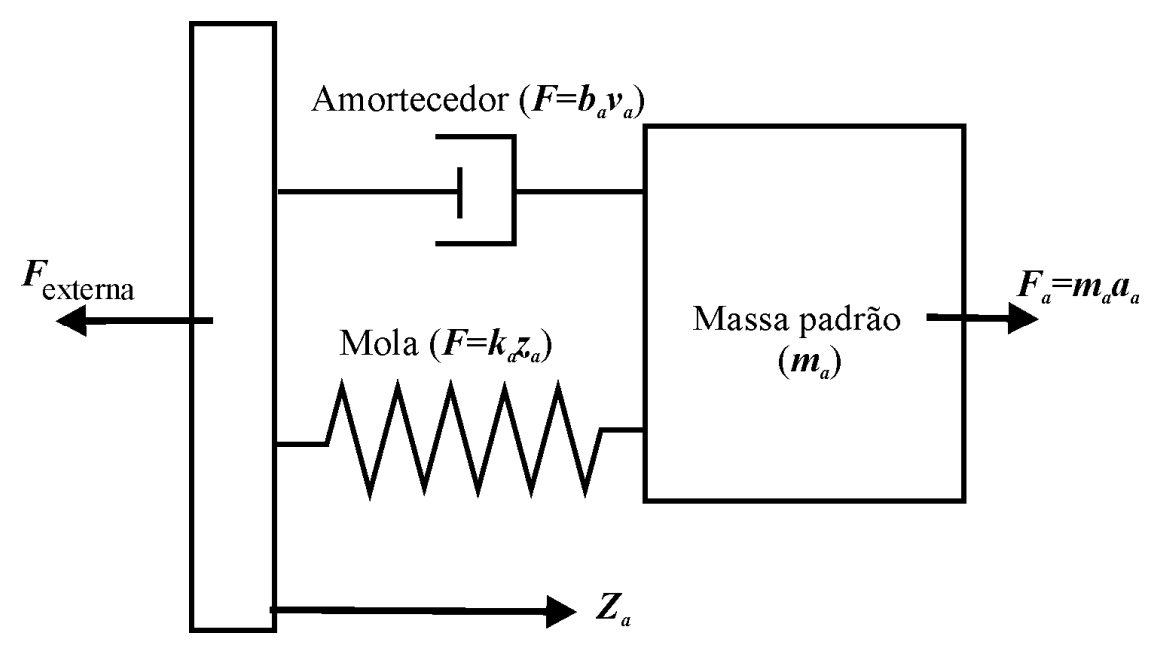

Figura 2.1: Esquema do sistema massa-mola-amortecedor.

Neste projeto foi utilizado o acelerômetro modelo ADXL105 do fabricante Analog Devices. Este sensor pode medir aceleração dinâmica (típica ou vibração) ou aceleração estática (força de inércia, gravidade ou inclinação). Neste trabalho o acelerômetro é usado para medir aceleração dinâmica.

O ADXL105 é um sistema completo de medida de aceleração, fabricado em um circuito integrado que combina o sensor mecânico e o circuito de condicionamento de sinal. O sistema massa-mola utilizado é do tipo MEMS 
("MicroElectroMechanical Systems") e complementarmente existe um sensor de posição por diferença capacitiva.

A figura 2.2 apresenta a vista superior esquemática do sensor. O eixo sensitivo para a aceleração está no plano do dispositivo. A massa formada de silício é sustentada por molas também de silício que proporcionam uma resistência contra a força induzida pela aceleração. A deformação da estrutura é medida com uma estrutura de capacitor diferencial, que consiste de duas placas fixas e independentes e uma placa central fixada na estrutura da massa móvel. A diferença de capacitância produz uma tensão de saída proporcional à aceleração.

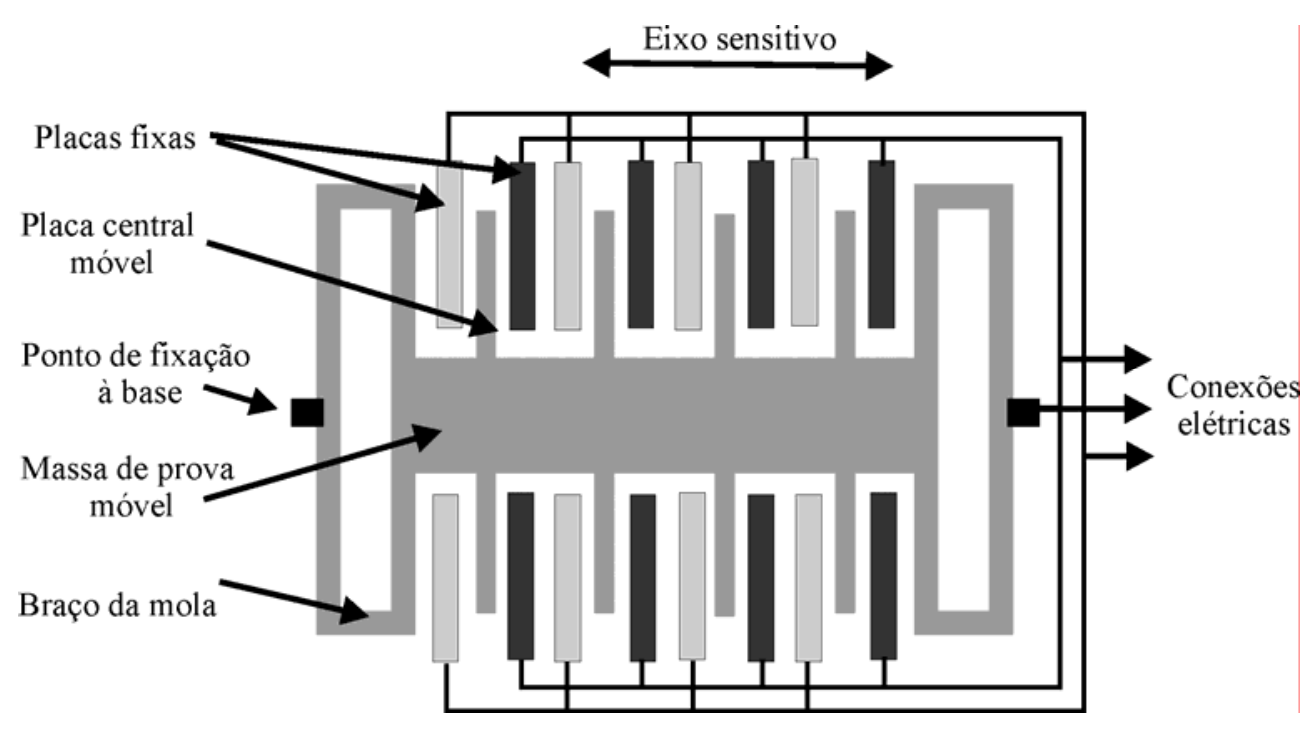

Figura 2.2: Vista esquemática superior do sensor.

$\mathrm{Na}$ figura 2.3 tem-se o diagrama do sensor em repouso e na figura 2.4, a resposta do sensor à aplicação de uma aceleração externa. $C 1$ e $C 2$ são valores de capacitância. 


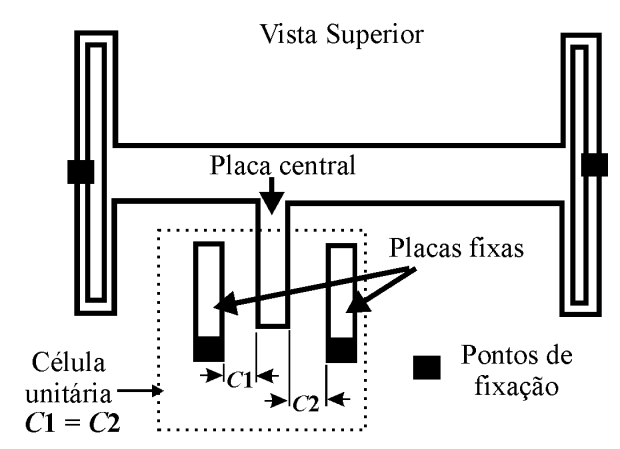

Figura 2.3: Diagrama do sensor em repouso.

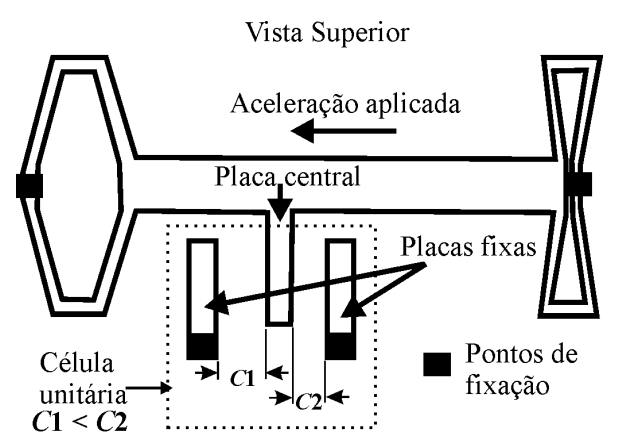

Figura 2.4: Resposta do sensor a uma aceleração externa.

\subsection{Descrição geral e características técnicas}

O acelerômetro utilizado, ADXL105, é um transdutor que mede a aceleração num único eixo. Os dados técnicos principais estão apresentados na tabela 2.1. Em cada canto do protótipo do veículo está instalado, ao longo do eixo vertical $z$, um acelerômetro.

Tabela 2.1: Dados técnicos do acelerômetro $\left(T_{\mathrm{A}}=25^{\circ} \mathrm{C}, V_{\mathrm{DD}}=5 \mathrm{~V}\right.$ e acel. $\left.=0 g\right)$.

\begin{tabular}{|l|l|}
\hline \multicolumn{1}{|c|}{ Parâmetros } & \multicolumn{1}{c|}{ Valores típicos } \\
\hline Range de medida & $+/-5 \mathrm{~g}$ \\
\hline Sensibilidade & $250 \mathrm{mV} / \mathrm{g}$ \\
\hline Ruído & $225 \mu \mathrm{g} / \sqrt{\mathrm{Hz}}$ \\
\hline Ruído com largura de banda de $10 \mathrm{kHz}$ & $22,5 \mathrm{mg} \mathrm{rms}$ \\
\hline Largura de banda & $10 \mathrm{kHz}$ \\
\hline Tensão de alimentação $\left(V_{\mathrm{DD}}\right)$ & 2,7 a $5,25 \mathrm{~V}$ \\
\hline Erro de offset para zero g & $2,5 \mathrm{~V}+/-625 \mathrm{mV}$ \\
\hline Temperatura de operação $\left(T_{\mathrm{A}}\right)$ & 0 a $70^{\circ} \mathrm{C}$ \\
\hline
\end{tabular}

Na tabela 2.1, a letra $g$ é a aceleração da gravidade, que neste projeto foi considerada como sendo $9,807 \mathrm{~m} / \mathrm{s}^{2}$.

A figura 2.5 fornece o circuito de aplicação desenvolvido para medir a aceleração. Neste podem-se tecer os seguintes comentários: 
a) Visto que a tensão de saída é diretamente proporcional à tensão de alimentação $\left(V_{\mathrm{DD}}\right)$, foram utilizados: um filtro $R-C$ passa-baixas para assegurar que $V_{\mathrm{DD}}$ não contenha ruído de alta freqüência e um circuito regulador de voltagem, veja anexo $\mathrm{A}$, que fornece uma tensão constante de $5 V \mathrm{cc}$ para alimentação $(V \mathrm{~s})$.

b) A sensibilidade da saída, que é de $0,25[\mathrm{~V} / \mathrm{g}]$ (tabela 2.1), pode ser ajustada para um valor maior com a utilização do amplificador operacional, interno ao circuito integrado do acelerômetro, e colocando-se externamente resistores.

Sendo $R_{1}=50 \mathrm{k} \Omega$ e $R_{2}=200 \mathrm{k} \Omega$ (veja figura 2.5), a sensibilidade teórica é dada por:

$$
\text { sensibilidade }=0,25 \frac{R_{2}}{R_{1}}\left[\frac{V}{g}\right] .
$$

Mas na prática, o valor real da sensibilidade foi ajustado através do seguinte procedimento:

- o eixo do acelerômetro foi apontado para o solo e lido o valor da tensão $\left(U_{+\mathrm{g}}\right)$ para $+1 g$;

- o eixo do sensor foi deslocado de $180^{\circ}$ e leu-se o valor da tensão $\left(U_{-\mathrm{g}}\right)$ para $-1 g$.

Utilizando-se destas duas leituras a sensibilidade real é dada por:

$$
\text { sensibilidade }=\frac{U_{+g}-U_{-g}}{2}\left[\frac{V}{g}\right] .
$$

A tabela 2.2 apresenta a sensibilidade real para cada um dos quatro acelerômetros. 
Tabela 2.2: Sensibilidade real dos acelerômetros.

\begin{tabular}{|c|c|c|c|}
\hline Acelerômetro & $U_{+\mathrm{g}}(V)$ & $U_{-\mathrm{g}}(V)$ & sensibilidade $[\mathrm{V} / \mathrm{g}]$ \\
\hline 1 & 3,38 & 1,62 & 0,88 \\
\hline 2 & 3,41 & 1,61 & 0,90 \\
\hline 3 & 3,37 & 1,61 & 0,88 \\
\hline 4 & 3,39 & 1,61 & 0,89 \\
\hline
\end{tabular}

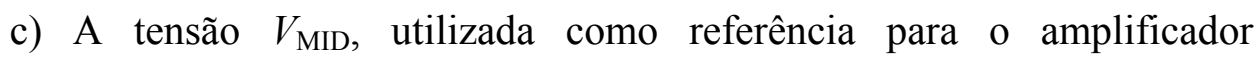
operacional, tem um valor nominal de $V_{\mathrm{DD}} / 2$.

d) O potenciômetro de $10 \mathrm{k} \Omega$ é utilizado para compensar o erro de offset e ajustar a tensão de saída do operacional (UCAOUT) para o valor de $V_{\mathrm{DD}} / 2=2,5 \mathrm{~V}$, quando a aceleração é de zero $g$. Neste projeto foi utilizada a tensão $2,5 \mathrm{~V}$ para $1 g$.

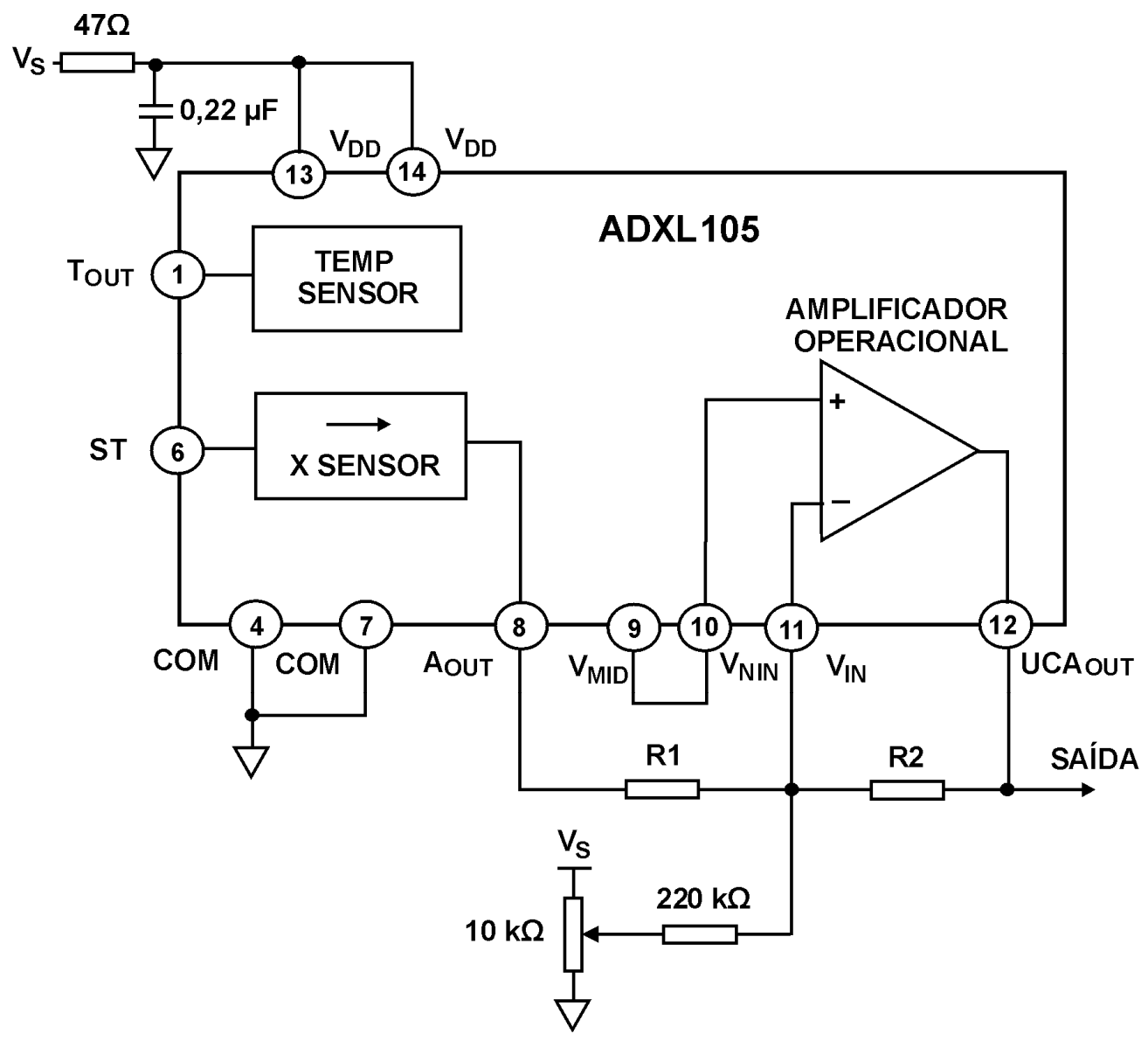

Figura 2.5: Circuito de aplicação desenvolvido. 


\subsection{Cálculo do valor de referência}

Quando o sistema for inicializado, amostras serão feitas com o sistema em repouso, e a média calculada será o valor de $1 g$, pois o acelerômetro está instalado no eixo $z$ e está sujeito à ação da gravidade.

A seguir está apresentada uma fórmula de recorrência que permite calcular o valor da média em função da média anterior e da leitura atual.

Sabendo-se que a média aritmética no instante atual $(\bar{x}(n))$ é dada por:

$$
\bar{x}(n)=\frac{\sum_{i=1}^{n} x_{i}}{n},
$$

então o valor da média aritmética no instante seguinte $(\bar{x}(n+1))$ é dado por:

$$
\bar{x}(n+1)=\frac{\sum_{i=1}^{n+1} x_{i}}{n+1}=\frac{\left(\sum_{i=1}^{n} x_{i}\right)+x_{n+1}}{n+1},
$$

onde $x_{i}$ são os valores lidos e $n$ a quantidade de valores lidos.

Substituindo-se a expressão (2.1) em (2.2), tem-se que:

$$
\begin{gathered}
\bar{x}(n+1)=\frac{n \bar{x}(n)+x_{n+1}}{n+1}=\frac{n \bar{x}(n)+\bar{x}(n)-\bar{x}(n)+x_{n+1}}{n+1} \Rightarrow \\
\bar{x}(n+1)=\bar{x}(n)+\frac{1}{n+1}\left(x_{n+1}-\bar{x}(n)\right) .
\end{gathered}
$$

Reescrevendo a equação (2.3) em função do valor de referência, tem-se:

$$
\operatorname{refAout}(n+1)=\operatorname{refAout}(n)+\frac{1}{n+1}\left(U C A_{\text {OUT }}(n+1)-\operatorname{refAout}(n)\right) .
$$

O cálculo do valor de referência, ou valor de tensão para aceleração inercial nula (refAout), é fornecido pela expressão (2.4), que está implementada em uma rotina desenvolvida em Pascal. 


\subsection{Cálculo da aceleração inercial}

O valor da aceleração inercial medida no eixo $z$ para os quatro acelerômetros $\ddot{z}_{m p}(t)(p=1, \ldots, 4)$ é dado por:

$$
\ddot{z}_{m p}(t)=\frac{\left(\text { refAout }_{p}-U C A_{\text {OUT }}(t)\right) g}{\text { sensibilidade }_{p}},
$$

e a unidade da aceleração é $m / s^{2}$.

\subsection{Cálculo da velocidade}

A idéia inicial para obtenção de velocidade foi realizar a integração direta do sinal de aceleração, mas quando da execução deste método, o sinal da velocidade apresentou uma deriva. Verifica-se, conforme apresentado no exemplo a seguir, que se existe uma componente contínua (DC) como resultado da calibração do valor de referência ou uma deriva térmica, o sinal da velocidade tende a divergir.

Este problema de deriva resolve-se com a utilização de um filtro passa-altas antes da integração para o cálculo da velocidade. Neste projeto, a velocidade estimada $\dot{z}_{p}(t)$ foi calculada com a passagem do sinal medido da aceleração $\ddot{z}_{m p}(t)$ por um filtro passa-altas, com freqüência de corte $w_{c}$, e um bloco integrador, conforme figura 2.6.

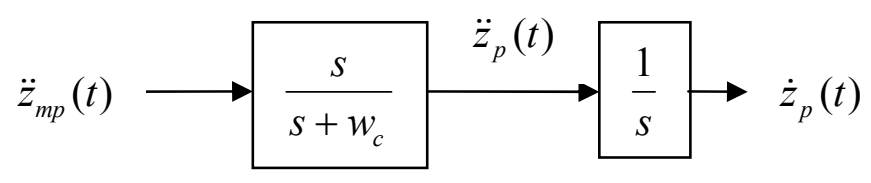

Figura 2.6: Diagrama de blocos em s (Laplace) para cálculo da velocidade.

A partir das funções de transferência no domínio de Laplace, o integrador e o filtro passa-altas foram discretizados, utilizando-se a aproximação de Tustin. 
As funções de transferência obtidas, no domínio discreto $(Z)$, com freqüência de amostragem Freq, são dadas, conforme figura 2.7, por:

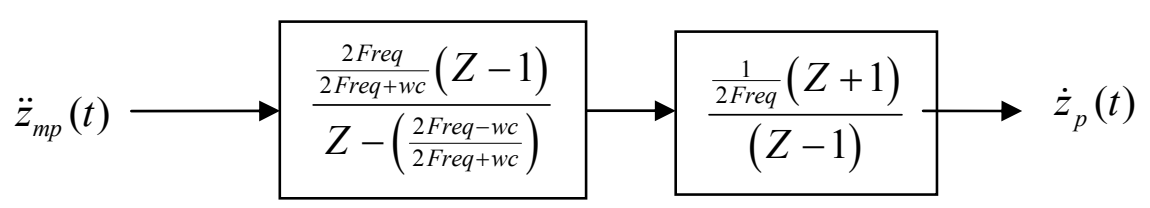

Figura 2.7: Diagrama de blocos em Z (discreto) para cálculo da velocidade.

Convencionando que $Z^{-1}$ é um operador de atraso, isto é:

$$
Z^{-1} f(k)=f(k-1)
$$

foi obtida a equação de diferenças para o filtro e integrador, que é dada por:

$$
\dot{z}_{p}(k)=\frac{2 \text { Freq }-w_{c}}{2 \text { Freq }+w_{c}} \dot{z}_{p}(k-1)+\frac{1}{2 \text { Freq }+w c}\left(\ddot{z}_{m p}(k)+\ddot{z}_{m p}(k-1)\right),
$$

onde $\ddot{z}_{m p}(k)$ e $\dot{z}_{p}(k)$ representam, respectivamente, a aceleração medida e a velocidade estimada para o acelerômetro $p(p=1, \ldots, 4)$ no instante $k$.

A figura 2.8 apresenta um exemplo de um sinal de aceleração com média nula e uma componente DC de $0,004 \mathrm{~m} / \mathrm{s}^{2}$ (corresponde a $0,5 \%$ do valor de pico). Verifica-se que o sinal da velocidade, obtido pela integração da aceleração (sem filtro), apresenta uma deriva com o tempo, mas o sinal da velocidade estimada com o filtro $\left(\right.$ Freq $=5000 \mathrm{~Hz}$ e $\left.w_{\mathrm{c}}=1 \mathrm{rad} / \mathrm{s}\right)$ tem uma deriva relativamente reduzida permanecendo próximo do valor zero. 


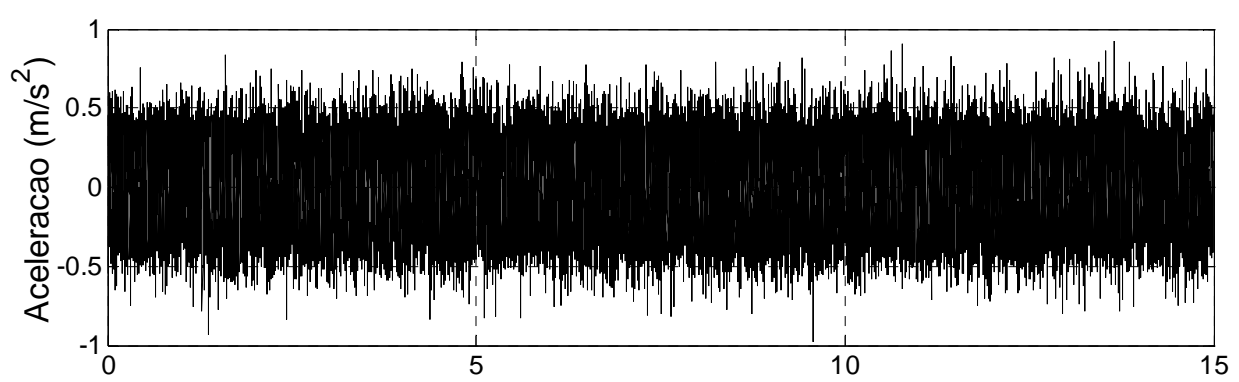

$t(s)$

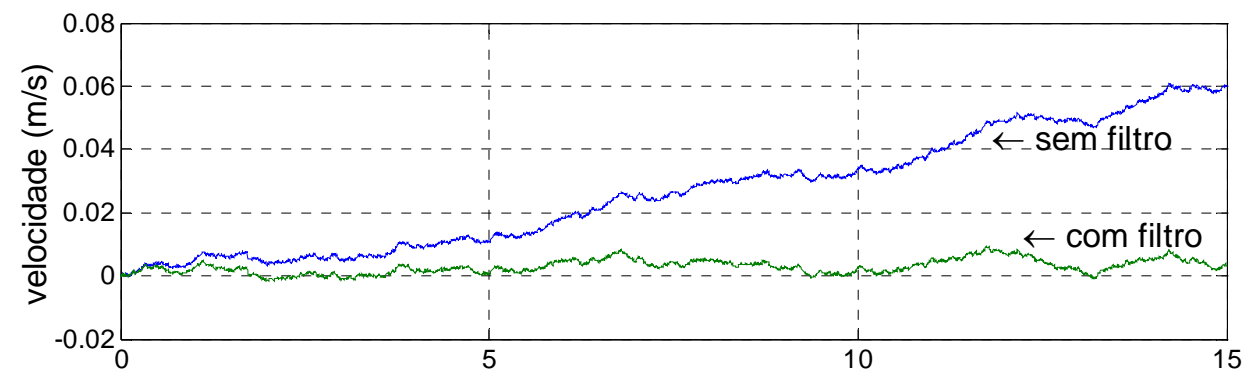

$\mathrm{t}(\mathrm{s})$

Figura 2.8: Aceleração com componente DC e velocidade com e sem filtro.

Conforme mostrado na figura 2.9, o aumento da freqüência de corte do filtro passa-altas de $1 \mathrm{rad} / \mathrm{s}$ para $10 \mathrm{rad} / \mathrm{s}$, leva a uma diminuição no valor da amplitude da velocidade, mas em contra partida, perdem-se as informações em baixa freqüência do sinal. 


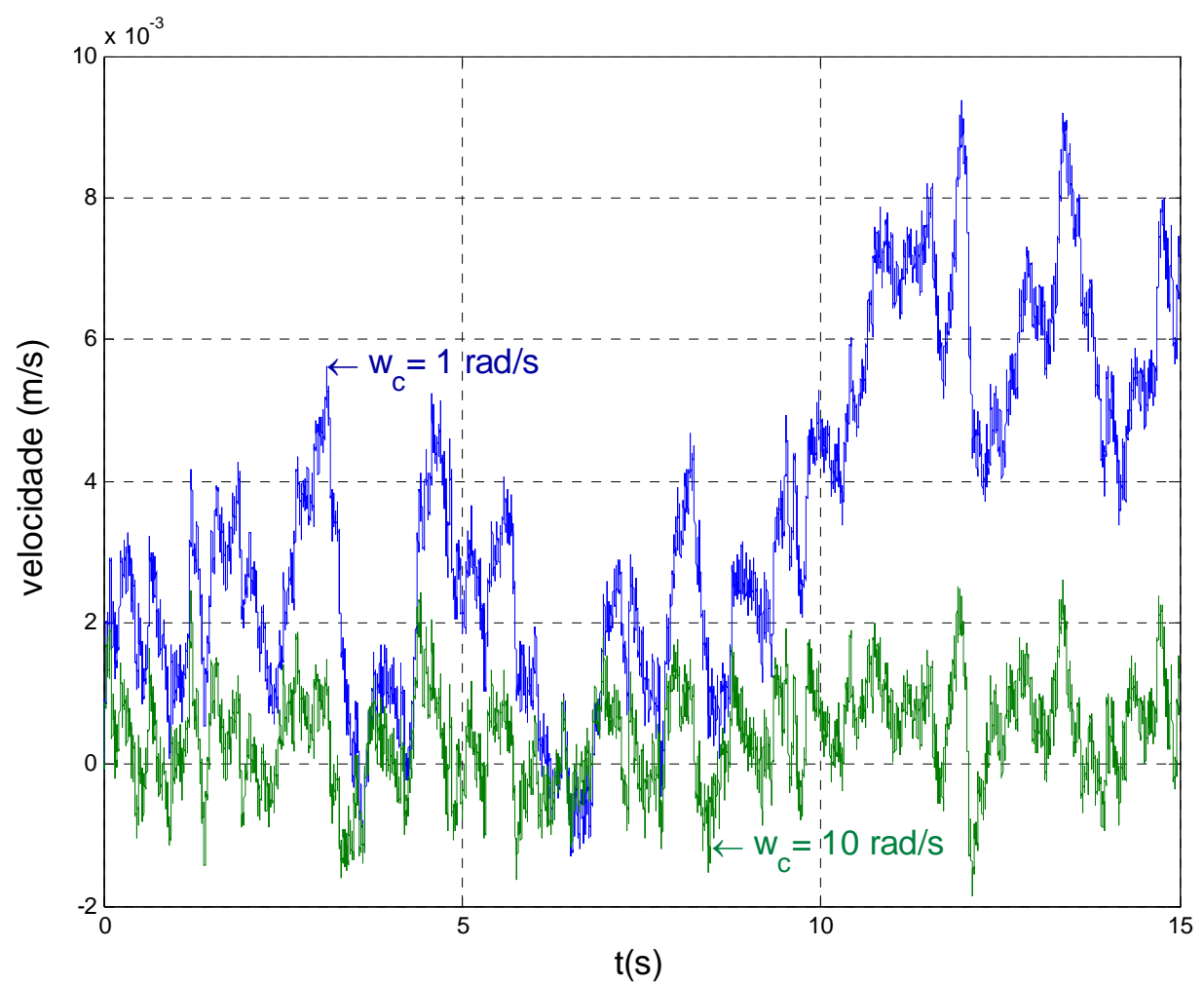

Figura 2.9: Velocidade com integrador e filtro passa-altas de $w_{c}=1 \mathrm{rad} / \mathrm{s}$ e $10 \mathrm{rad} / \mathrm{s}$.

Uma dificuldade deste projeto foi a escolha de uma freqüência de corte adequada para o filtro passa-altas. Sabendo-se que o protótipo apresenta freqüências de ressonância predominantes em 10 rad/s e 40rad/s (BITTAR,1998), então, escolheu-se inicialmente uma freqüência de corte de $10 \mathrm{r} a d / s$. Após a operação do sistema alterou-se a freqüência de corte para um valor melhor, neste caso $w_{c}=$ $8 \mathrm{rad} / \mathrm{s}$. 


\section{Capítulo 3}

\section{MODELO MATEMÁTICO DO SISTEMA E COMPATIBILIZAÇÃO}

Neste capítulo define-se o modelo matemático do sistema e apresenta-se a compatibilização das variáveis do veículo com as variáveis do modelo matemático do sistema, através dos blocos "Comando de Atuadores" e "Processamento de Medidas".

\subsection{Modelo matemático do sistema}

Os controladores que realizam a levitação eletromagnética do veículo foram desenvolvidos considerando três modelos matemáticos diferentes. Os modelos são:

- modelo de corpo rígido;

- modelo de corpo não rígido;

- modelo SISO.

A figura 3.1 apresenta o esquema das forças aplicadas no protótipo. Sendo que:

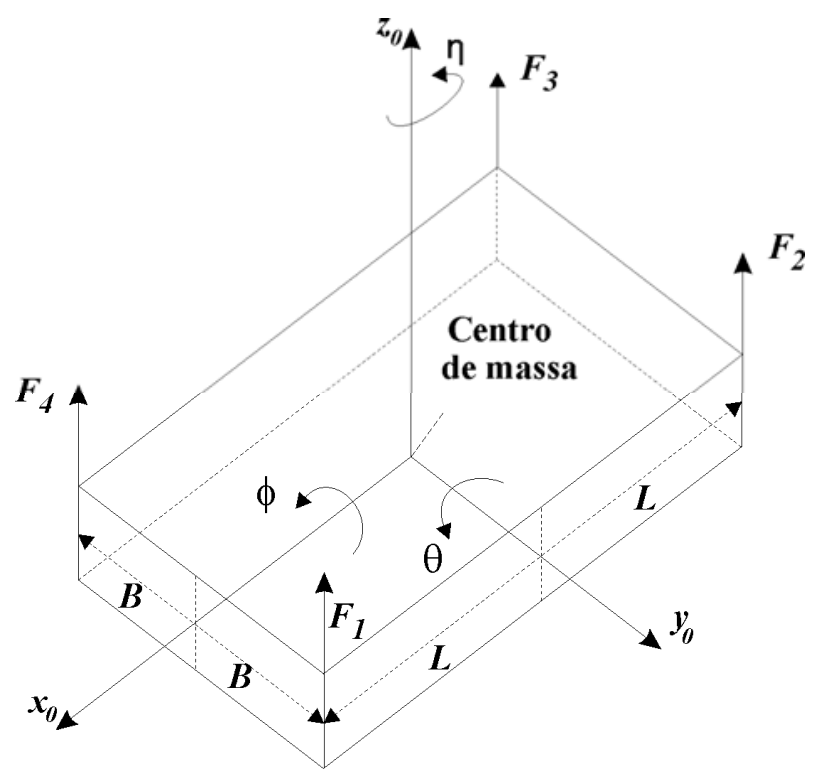

Figura 3.1: Esquema de forças de suspensão. 
$F_{1}, F_{2}, F_{3}$ e $F_{4}$ são as forças produzidas pelos eletroímãs $1,2,3$ e 4 respectivamente;

$\left(x_{0}, y_{0}, z_{0}\right)$ representa o sistema de coordenadas fixo no veículo;

$B$ e $L$ são as distâncias dos centros dos eletroímãs até os eixos $x_{0}$ e $y_{0}$, respectivamente $(15,75 \mathrm{~cm}$ e $25,65 \mathrm{~cm})$.

Na figura 3.2 têm-se os possíveis movimentos que podem ser realizados pelo protótipo durante a levitação: movimento vertical $(z)$, de rolagem $(\phi)$, de arfagem $(\theta)$ e de torção $(\xi)$, este último para o caso não rígido.
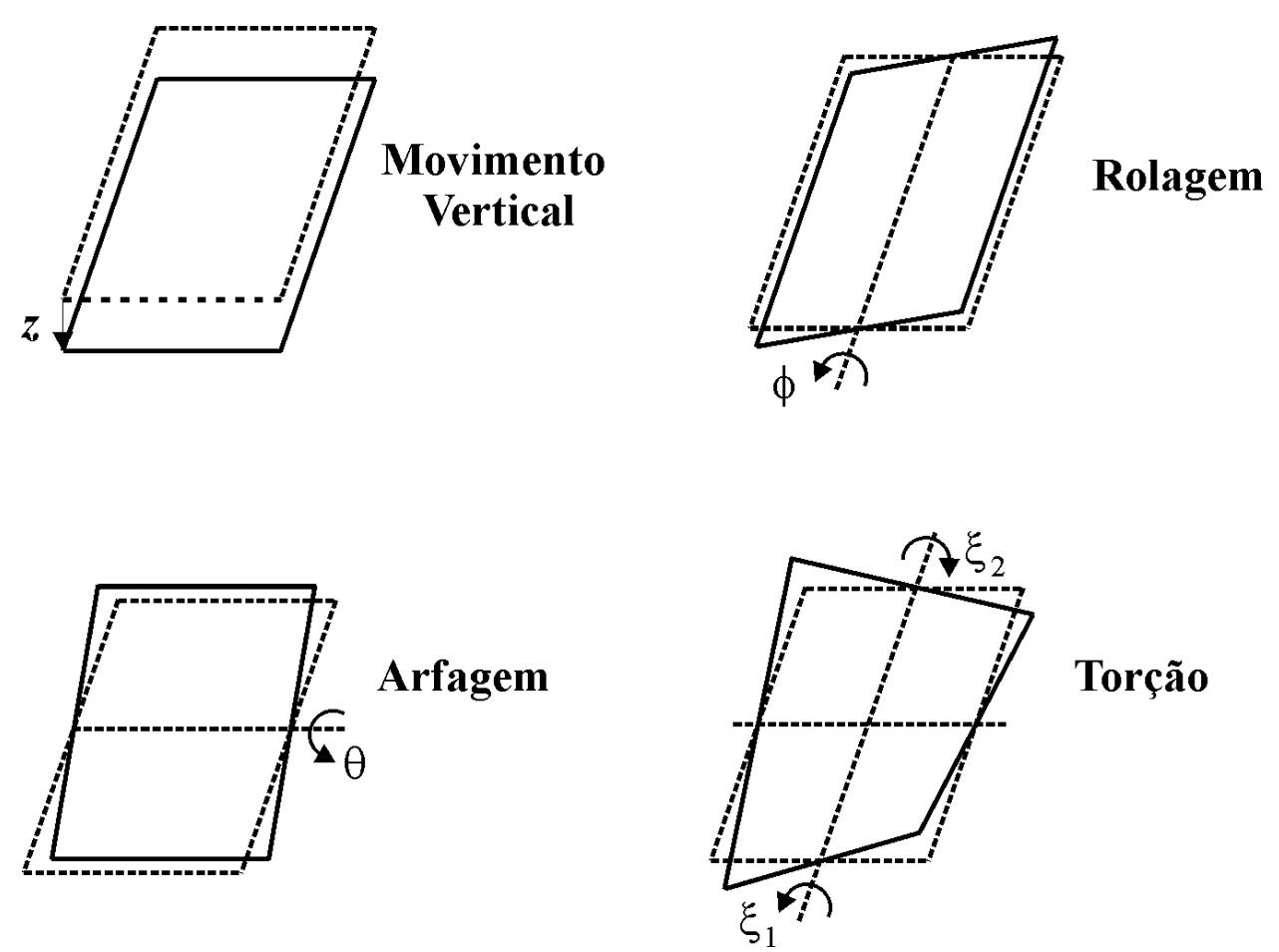

Figura 3.2: Movimentos do veículo.

A figura 3.3 apresenta em detalhe o movimento vertical com as forças atuantes e orientação da medida do entreferro. Sendo que:

$F_{z}(t)$ é a força vertical resultante;

$z(t)$ é a medida do entreferro;

$M$ é a massa total do veículo com carga $(97 \mathrm{~kg})$; 
$g$ é a aceleração local da gravidade.

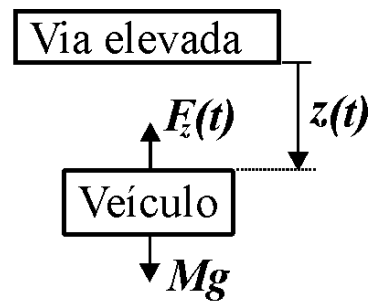

Figura 3.3: Detalhe do movimento vertical.

A seguir, para cada um dos modelos são apresentadas as equações que descrevem a dinâmica do veículo e a compatibilização das suas variáveis.

\subsubsection{Modelo de corpo rígido}

Considerando-se que o corpo é rígido, ou seja, não existe movimento de torção e que os movimentos de rotação são de pequena amplitude, escreve-se, de maneira direta, a dinâmica do movimento do veículo representado nas figuras 3.1, 3.2 e 3.3. As equações do modelo são:

$$
\begin{gathered}
M \ddot{z}(t)=M g-F_{z}(t), \\
I_{x x} \ddot{\phi}(t)=M_{x}(t), \\
I_{y y} \ddot{\theta}(t)=M_{y}(t),
\end{gathered}
$$

onde:

$M_{x}(t)$ e $M_{y}(t)$ são as componentes dos momentos aplicados segundo os eixos $x_{0}$ e $y_{0}$, respectivamente;

$I_{x x}$ e $I_{y y}$ são os correspondentes momentos de inércia relativos aos eixos $x_{0} \mathrm{e}$ $y_{0}$, respectivamente $\left(5,9156 \mathrm{kgm}^{2}\right.$ e $\left.7,9287 \mathrm{kgm}^{2}\right)$. 
Este modelo dinâmico baseia-se em (SINHA, 1987) e (BITTAR, 1998).

Nota-se que o modelo tem a forma de um sistema desacoplado com equações lineares, onde os movimentos vertical, de rolagem e de arfagem são dados, respectivamente, pelas equações (3.1), (3.2) e (3.3).

\subsubsection{Modelo de corpo não rígido}

Considerando-se que o corpo não é rígido, ou seja, o movimento de torção não pode ser desprezado, escreve-se de maneira direta (BITTAR,1998), a dinâmica do movimento do veículo representado nas figuras 3.1, 3.2 e 3.3. As equações do modelo são:

$$
\begin{gathered}
M \ddot{z}(t)=M g-F_{z}(t), \\
I_{x x} \ddot{\phi}(t)=M_{x}(t), \\
I_{y y} \ddot{\theta}(t)=M_{y}(t), \\
I_{t} \ddot{\xi}(t)=M_{t},
\end{gathered}
$$

onde:

$M_{t}(t)$ é a torção no eixo $x_{0}$;

$I_{t}$ é o momento de inércia com relação ao eixo $x_{0}$. Conforme apresentado em (BITTAR, 1998), pode-se considerar que $I_{t} \cong I_{x x}$;

$\xi(t)$ é o ângulo de torção no eixo $x_{0}$.

O modelo tem a forma de um sistema desacoplado com equações lineares, onde os movimentos: vertical, de rolagem, de arfagem e de torção são dados, respectivamente, pelas equações (3.4), (3.5), (3.6) e (3.7). 


\subsubsection{Modelo SISO}

A fim de realizar uma abordagem simples, considera-se o sistema global como sendo composto por quatro sistemas SISO desacoplados, equivalentes e com a mesma dinâmica que relaciona a força no atuador com o entreferro correspondente.

A seguir é apresentada a equação que descreve o sistema SISO equivalente:

$$
\frac{M}{4} \ddot{z}_{p}(t)=\frac{M}{4} g-F_{i}(t) \quad(i=p=1, \ldots, 4)
$$

onde:

$F_{i}(t)(i=1, \ldots, 4)$ são as forças verticais produzidas pelos eletroímãs e que agem em cada um dos cantos do veículo;

$\ddot{z}_{p}(t)(p=1, \ldots, 4)$ são as acelerações em cada um dos cantos do protótipo.

\subsection{Compatibilização das variáveis}

Blocos de compatibilização de variáveis são utilizados devido à diferença no número de variáveis de entrada e saída do modelo matemático com o número de variáveis de atuação e medidas, disponíveis no veículo.

Conforme apresentado na figura 3.4, o bloco de "Comando de Atuadores" faz a compatibilização das variáveis de entrada do modelo de corpo rígido $\left(F_{\mathrm{z}}(t), M_{\mathrm{x}}(t)\right.$ e $\left.M_{\mathrm{y}}(t)\right)$, que são as variáveis da saída dos controladores, com as variáveis de atuação do veículo $\left(v_{i}(t), i=1, \ldots, 4\right)$, que são as tensões aplicadas no circuito de potência. $\mathrm{O}$ bloco de "Processamento de Medidas" estima as variáveis de posição e velocidade, ou variáveis de estado do modelo $(z(t), \phi(t), \theta(t), \dot{z}(t), \dot{\phi}(t) e \dot{\theta}(t))$, a partir das quatro medidas de entreferro e das quatro de acelerações $\left(z_{p}(t), \ddot{z}_{p}(t), p=1, \ldots, 4\right)$, disponíveis em cada canto do protótipo. 


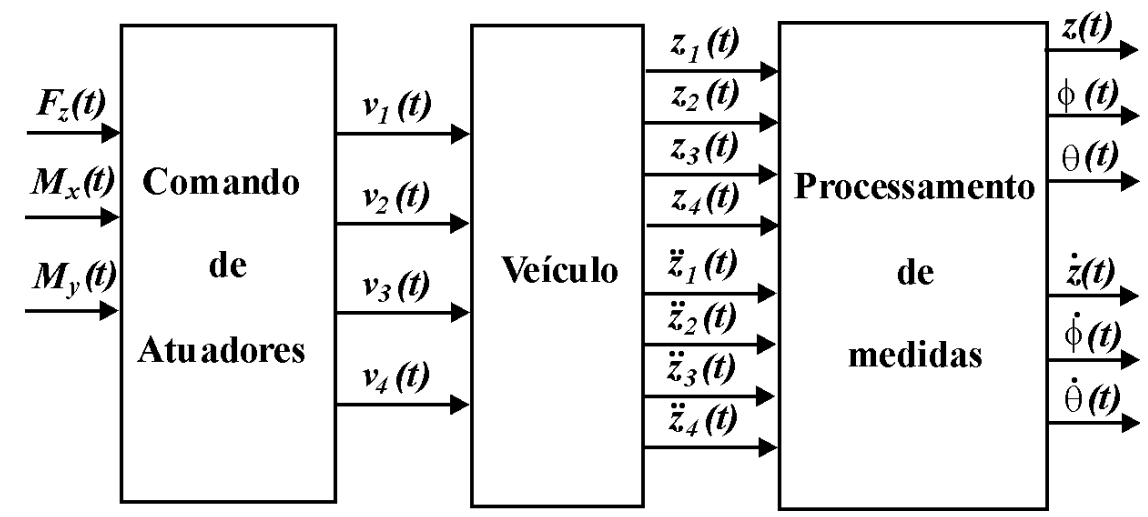

Figura 3.4: Blocos de compatibilização das variáveis do modelo de corpo rígido.

O detalhamento dos blocos "Comando de Atuadores" e "Processamento de Medidas” está apresentado nas seções seguintes.

No caso da compatibilização do modelo de corpo não rígido, figura 3.5, esta é similar ao modelo de corpo rígido com a inclusão do momento de torção $M_{\mathrm{t}}(t)$ na entrada do bloco "Comando de Atuadores" e das variáveis de estado $\xi(t) e \dot{\xi}(t)$ no bloco "Processamento de Medidas".

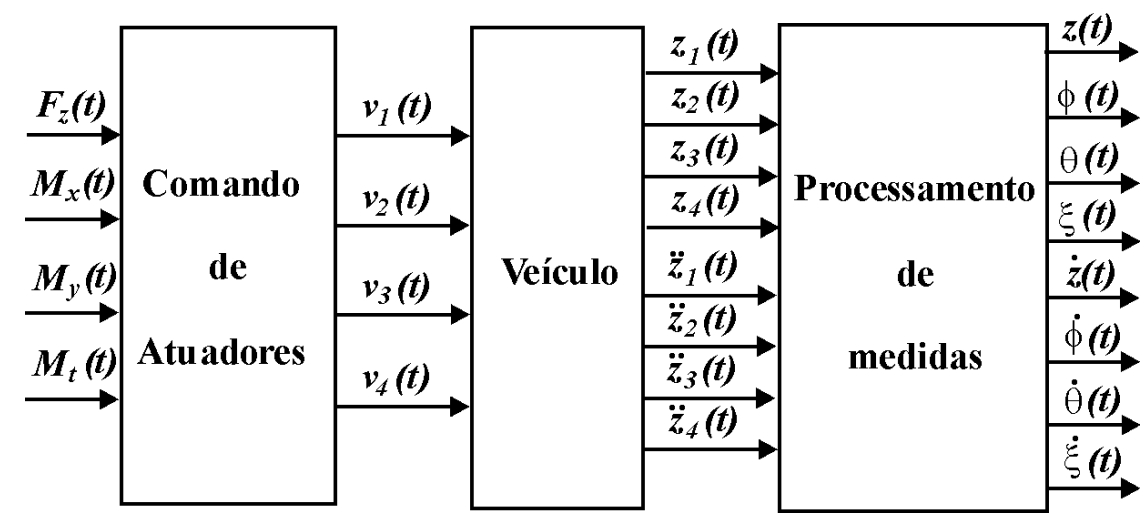

Figura 3.5: Blocos de compatibilização das variáveis do modelo de corpo não rígido.

$\mathrm{Na}$ compatibilização das variáveis do modelo SISO, figura 3.6, o bloco "Comando de Atuadores" recebe as forças $\left(F_{i}(t)(i=1, \ldots, 4)\right)$ e o bloco "Processamento de Medidas" tem como saída as variáveis de estado $\left(z_{p}(t), \dot{z}_{p}(t),(p=1, \ldots, 4)\right)$. 


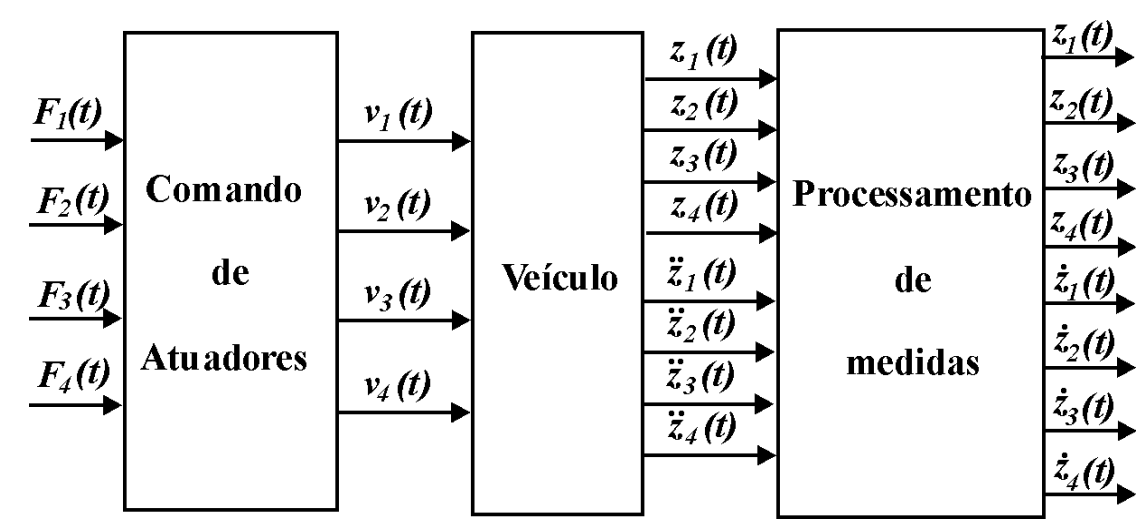

Figura 3.6: Blocos de compatibilização das variáveis do modelo SISO.

Considerando no modelo de corpo rígido que as variáveis de saída são apenas $z(t), \phi(t) e \theta(t)$, a figura 3.7 apresenta os blocos de compatibilização das variáveis.

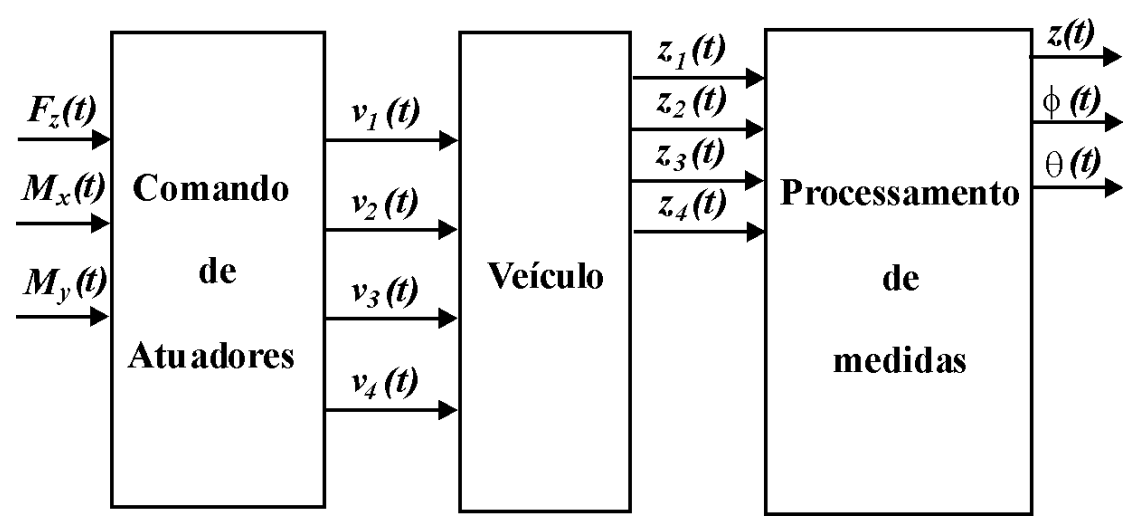

Figura 3.7: Detalhamento do modelo de corpo rígido com realimentação da saída.

\subsection{Bloco de comando de atuadores}

No bloco de "Comando de Atuadores", para cada um dos modelos considerados, são calculadas as forças eletromagnéticas de suspensão e as tensões que são aplicadas na entrada do circuito de potência do veículo.

\subsubsection{Relação da força eletromagnética com tensão elétrica}

Na saída do bloco de "Comando de Atuadores" têm-se as tensões $v_{1}(\mathrm{t}), v_{2}(\mathrm{t})$, $v_{3}(\mathrm{t})$ e $v_{4}(\mathrm{t})$, que são aplicadas na entrada do circuito de potência. 
Conforme apresentado no Anexo $\mathrm{C}$ as forças eletromagnéticas $F_{i}(t)$ são dadas por:

$$
F_{i}(t)=k_{v i}\left(\frac{v_{i}(t)}{z_{i}(t)}\right)^{2},
$$

e as tensões $v_{i}(t)$ são dadas por:

$$
v_{i}(t)=z_{i}(t) \sqrt{\frac{F_{i}(t)}{k_{v i}}},
$$

sendo :

$z_{\mathrm{i}}(\mathrm{t})$ : distância do entreferro;

$k_{\mathrm{vi}}$ : constante de proporcionalidade, cujo valor encontra-se no Anexo C;

$i=1, \ldots, 4$.

\subsubsection{Otimização das forças de suspensão para o modelo de corpo rígido}

No bloco "Comando de Atuadores", onde se considera o modelo como sendo de corpo rígido, são calculadas as forças eletromagnéticas $F_{1}(\mathrm{t}), F_{2}(\mathrm{t}), F_{3}(\mathrm{t})$ e $F_{4}(\mathrm{t})$ (ou as tensões $v_{1}(\mathrm{t}), v_{2}(\mathrm{t}), v_{3}(\mathrm{t})$ e $v_{4}(\mathrm{t})$ ), de acordo com as três variáveis de controle $\left(F_{\mathrm{z}}(\mathrm{t}), M_{\mathrm{x}}(\mathrm{t})\right.$ e $\left.M_{\mathrm{y}}(\mathrm{t})\right)$ requeridas pelo sistema. A indeterminação presente neste problema é removida por um processo de otimização.

Definindo $F_{u}(t)$ como o limite superior das forças eletromagnéticas:

$$
F_{u}(t) \geq F_{1}(t), F_{2}(t), F_{3}(t), F_{4}(t)
$$

o objetivo é minimizar $F_{u}(t)$ sujeita às restrições abaixo. 
Da figura 3.1, para ângulos $\phi(t)$ e $\theta(t)$ suficientemente pequenos, pode ser escrito que:

$$
\begin{gathered}
F_{z}(t)=F_{1}(t)+F_{2}(t)+F_{3}(t)+F_{4}(t), \\
M_{x}(t)=\left(F_{1}(t)+F_{2}(t)-F_{3}(t)-F_{4}(t)\right) B, \\
M_{y}(t)=\left(-F_{1}(t)+F_{2}(t)+F_{3}(t)-F_{4}(t)\right) L .
\end{gathered}
$$

Sabendo-se que os eletroímãs geram apenas forças de atração, tem-se que:

$$
F_{1}(t), F_{2}(t), F_{3}(t), F_{4}(t) \geq 0
$$

O problema de minimização de $F_{\mathrm{u}}(t)$, sujeita às restrições das equações (3.11)(3.15), é uma forma de problema de programação linear, cuja solução pode ser obtida analiticamente.

Das equações (3.12)-(3.14) segue que:

$$
\left[\begin{array}{ccc}
1 & 1 & 1 \\
1 & 1 & -1 \\
-1 & 1 & 1
\end{array}\right]\left[\begin{array}{l}
F_{1}(t) \\
F_{2}(t) \\
F_{3}(t)
\end{array}\right]=\left[\begin{array}{c}
F_{z}(t)-F_{4}(t) \\
\frac{M_{x}(t)}{B}+F_{4}(t) \\
\frac{M_{y}(t)}{L}+F_{4}(t)
\end{array}\right]
$$

Resolvendo o sistema (3.16) com relação a $F_{1}(\mathrm{t}), F_{2}(\mathrm{t})$ e $F_{3}(\mathrm{t})$, tem-se que:

$$
\begin{gathered}
F_{1}(t)=a_{1}-F_{4}(t), \\
F_{2}(t)=a_{2}+F_{4}(t), \\
F_{3}(t)=a_{3}-F_{4}(t),
\end{gathered}
$$

onde: 


$$
\begin{gathered}
a_{1} \square \frac{1}{2}\left(F_{z}(t)-M_{y}(t) / L\right), \\
a_{2} \square \frac{1}{2}\left(M_{x}(t) / B+M_{y}(t) / L\right), \\
a_{3} \square \frac{1}{2}\left(F_{z}(t)-M_{x}(t) / B\right),
\end{gathered}
$$

Note-se que, devido as características físicas do sistema e sendo as forças eletromagnéticas apenas de atração, equação (3.15), então:

$$
\begin{aligned}
& \text { i) } F_{z} \geq 0 \\
& \text { ii) }\left|\frac{M_{x}}{B}+\frac{M_{y}}{L}\right| \leq F_{z} \Leftrightarrow\left|\frac{M_{x}}{B}\right|+\left|\frac{M_{y}}{L}\right| \leq F_{z} .
\end{aligned}
$$

Logo as equações (3.20) - (3.23) têm-se os seguintes sinais:

$$
\begin{aligned}
& \text { i) } a_{1} \geq 0 \\
& \text { ii) } a_{2} \text { indefinido; } \\
& \text { iii) } a_{3} \geq 0
\end{aligned}
$$

Utilizando as equações (3.17)-(3.19), as restrições (3.15) são escritas em termos de $F_{4}(\mathrm{t})$, produzindo:

$$
\left.\begin{array}{l}
F_{2}(t) \geq 0 \Leftrightarrow F_{4}(t) \geq-a_{2} \\
F_{4}(t) \geq 0
\end{array}\right\} \Leftrightarrow F_{4}(t) \geq \max \left\{0,-a_{2}\right\} \square b_{1} \geq 0
$$

$\mathrm{e}$

$$
\left.\begin{array}{l}
F_{1}(t) \geq 0 \Leftrightarrow F_{4}(t) \leq a_{1} \\
F_{3}(t) \geq 0 \Leftrightarrow F_{4}(t) \leq a_{3}
\end{array}\right\} \Leftrightarrow F_{4}(t) \leq \min \left\{a_{1}, a_{3}\right\} \square b_{2} \geq 0 .
$$

As restrições (3.24) e (3.25) serão reescritas como:

$$
\begin{aligned}
& F_{4}(t) \geq b_{1}, \\
& F_{4}(t) \leq b_{2} .
\end{aligned}
$$


Também das equações (3.17)-(3.19), as restrições (3.11) podem ser escritas em termos de $F_{4}(\mathrm{t})$ e $F_{\mathrm{u}}(\mathrm{t})$, produzindo:

$$
\left.\begin{array}{l}
F_{1}(t) \leq F_{u}(t) \Leftrightarrow a_{1}-F_{4}(t) \leq F_{u}(t) \Leftrightarrow F_{4}(t)+F_{u}(t) \geq a_{1} \\
F_{3}(t) \leq F_{u}(t) \Leftrightarrow a_{3}-F_{4}(t) \leq F_{u}(t) \Leftrightarrow F_{4}(t)+F_{u}(t) \geq a_{3}
\end{array}\right\} \Leftrightarrow
$$

e

$$
\begin{aligned}
& F_{2}(t) \leq F_{u}(t) \Leftrightarrow a_{2}+F_{4}(t) \leq F_{u}(t) \Leftrightarrow F_{4}(t)-F_{u}(t) \leq-a_{2} \\
& F_{4}(t) \leq F_{u}(t) \Leftrightarrow F_{4}(t)-F_{u}(t) \leq 0 \\
& \Leftrightarrow F_{4}(t)-F_{u}(t) \leq \min \left\{0,-a_{2}\right\} \square b_{4} \leq 0
\end{aligned} \Leftrightarrow \Leftrightarrow
$$

As expressões (3.28) e (3.29) podem ser reescritas respectivamente como :

$$
\begin{gathered}
F_{u}(t) \geq-F_{4}(t)+b_{3}, \\
F_{u}(t) \geq F_{4}(t)-b_{4} \Leftrightarrow F_{u}(t) \geq F_{4}(t)+\left(-b_{4}\right) .
\end{gathered}
$$

A figura 3.8 ilustra as restrições (3.26), (3.27), (3.30) e (3.31) sobre $F_{u}(\mathrm{t})$ e $F_{4}(\mathrm{t})$.

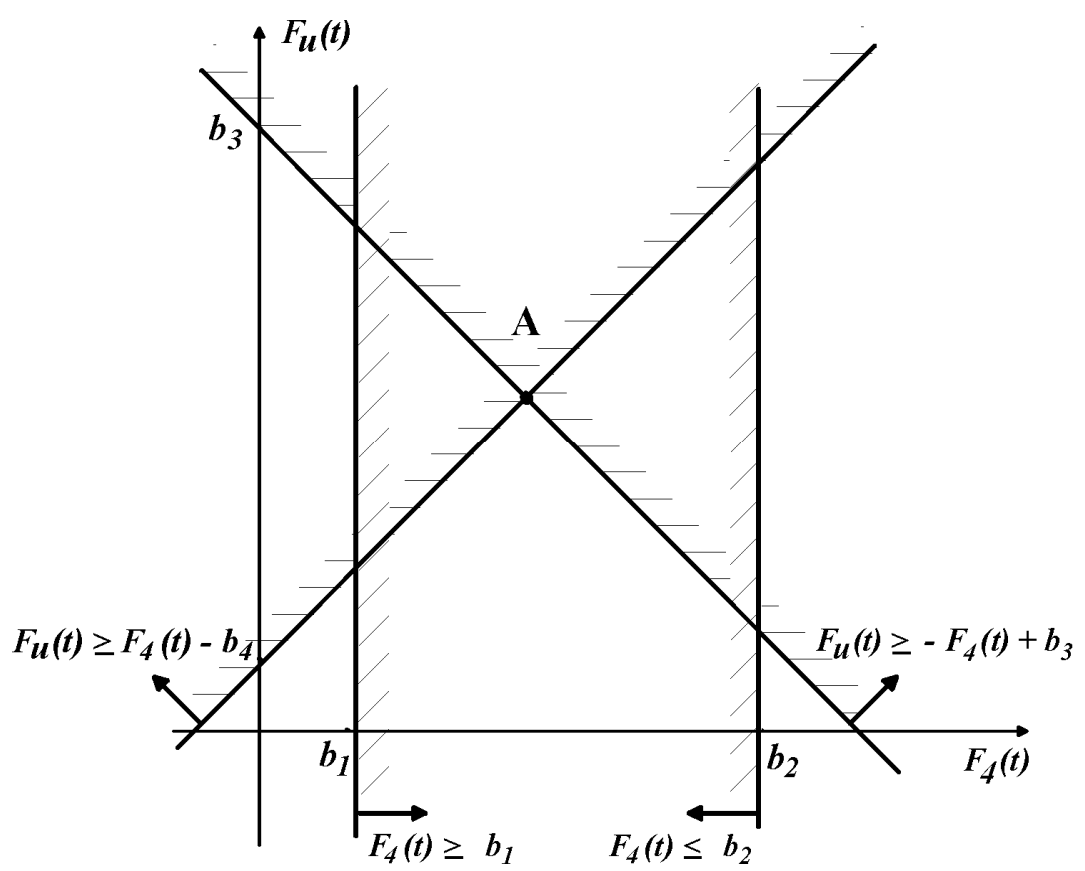

Figura 3.8: Restrições sobre $F_{\mathrm{u}}(\mathrm{t})$ e $F_{4}(\mathrm{t})$. 
O objetivo é minimizar $F_{\mathrm{u}}(\mathrm{t})$, na figura representada pelo ponto “A”, $\left(\frac{b_{3}+b_{4}}{2}\right.$ no eixo $\left.F_{4}(\mathrm{t})\right)$.

Da análise da figura 3.8 e das restrições impostas, tem-se a solução ótima $F_{4}^{*}(t)$, conforme segue:

$$
\begin{gathered}
\frac{b_{3}+b_{4}}{2}<b_{1} \Rightarrow F_{4}^{*}(t)=b_{1} ; \\
b_{1} \leq \frac{b_{3}+b_{4}}{2} \leq b_{2} \Rightarrow F_{4}^{*}(t)=\frac{b_{3}+b_{4}}{2} ; \\
\frac{b_{3}+b_{4}}{2}>b_{2} \Rightarrow F_{4}^{*}(t)=b_{2} .
\end{gathered}
$$

Substituindo a força $F_{4}^{*}(t)$ nas equações (3.17)-(3.19) encontramos as forças eletromagnéticas $F_{1}^{*}(t), F_{2}^{*}(t)$ e $F_{3}^{*}(t)$, e o problema está resolvido.

Proposição 3.1: Este problema de otimização sempre tem solução, pois tem-se sempre que $b_{1} \leq b_{2}$.

Vamos provar a proposição dividindo-a em duas partes:

Proposição 3.1.1: O problema de otimização tem solução se e somente se $b_{1} \leq b_{2}$.

Demonstração:

1) $b_{1} \leq b_{2} \Rightarrow$ tem solução.

Demonstra-se construindo a solução, conforme feito anteriormente.

2) tem solução $\Rightarrow b_{1} \leq b_{2}$.

Por absurdo, supõe-se que $b_{1}>b_{2}$, mas o problema de otimização tem solução.

Se o problema de otimização tem solução, então: 


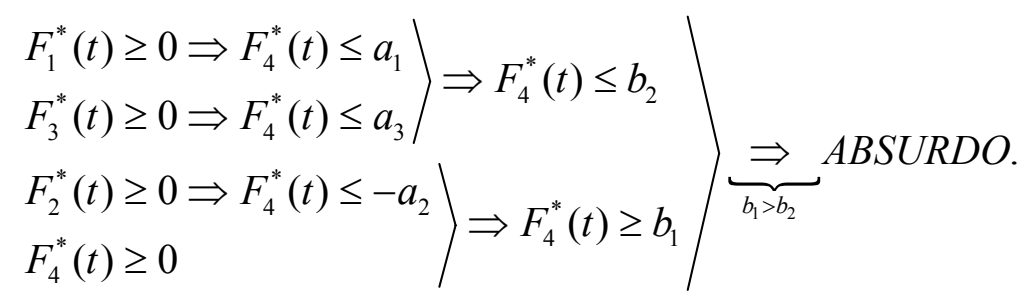

Proposição 3.1.2: Sempre tem-se que $b_{1} \leq b_{2}$.

Demonstração:

Por absurdo, supõe-se que $b_{1}>b_{2}$, então:

$\max \left\{0,-a_{2}\right\}>\min \left\{a_{1}, a_{3}\right\}$, para que tenha solução:

$$
\left\{\begin{array}{l}
-a_{2}>a_{1} \Leftrightarrow-\frac{1}{2}\left(\frac{M_{x}}{B}+\frac{M_{y}}{L}\right)>\frac{1}{2}\left(F_{z}-\frac{M_{y}}{L}\right) \Leftrightarrow-\frac{M_{x}}{B}>F_{z} \\
\text { ou } \\
-a_{2}>a_{3} \Leftrightarrow-\frac{1}{2}\left(\frac{M_{x}}{B}+\frac{M_{y}}{L}\right)>\frac{1}{2}\left(F_{z}-\frac{M_{x}}{B}\right) \Leftrightarrow-\frac{M_{y}}{B}>F_{z} .
\end{array}\right.
$$

Conforme a equação (3.23) o sistema não tem solução.

\section{Considerações}

Esta forma de distribuir os esforços pelos eletroímãs pode ser mais adequada do que aquela empregada no trabalho (BITTAR,1998), onde a função custo a ser minimizada é dada por uma função quadrática $J(t)=F_{1}^{2}(t)+F_{2}^{2}(t)+F_{3}^{2}(t)+F_{4}^{2}(t)$.

Aqui, a medida está relacionada diretamente às forças, e mais do que isso, o que se faz é minimizar a máxima dentre as quatro forças.

No problema de minimização proposto neste trabalho, resulta que a máxima força dentre as quatro $F_{i}(t)(i=1, \ldots, 4)$ é minimizada e, portanto, a estratégia de distribuição dos esforços tende a fazer com que os eletroímãs operem o mais distante possível da saturação. 


\subsubsection{Distribuição das forças para o modelo de corpo não rígido}

No bloco "Comando de Atuadores", onde se considera o modelo de corpo não rígido, ou seja, a torção do carro não pode ser desprezada, são calculadas as forças eletromagnéticas $F_{1}(\mathrm{t}), F_{2}(\mathrm{t}), F_{3}(\mathrm{t})$ e $F_{4}(\mathrm{t})$ (ou tensões $v_{1}(\mathrm{t}), v_{2}(\mathrm{t}), v_{3}(\mathrm{t})$ e $v_{4}(\mathrm{t})$ ), de acordo com as quatro variáveis de controle $\left(F_{\mathrm{z}}(\mathrm{t}), M_{\mathrm{x}}(\mathrm{t}), M_{\mathrm{y}}(\mathrm{t})\right.$ e $\left.M_{\mathrm{t}}(\mathrm{t})\right)$ requeridas pelo sistema. A solução neste caso será por um processo direto, conforme descrito.

Da figura 3.1, para ângulos $\phi(t), \theta(t)$ e $\xi(t)$ suficientemente pequenos, pode ser escrito que:

$$
\begin{gathered}
F_{z}(t)=F_{1}(t)+F_{2}(t)+F_{3}(t)+F_{4}(t), \\
M_{x}(t)=\left(F_{1}(t)+F_{2}(t)-F_{3}(t)-F_{4}(t)\right) B, \\
M_{y}(t)=\left(-F_{1}(t)+F_{2}(t)+F_{3}(t)-F_{4}(t)\right) L, \\
M_{t}(t)=\left(F_{1}(t)-F_{2}(t)+F_{3}(t)-F_{4}(t)\right) B .
\end{gathered}
$$

Devido às características físicas do sistema e sendo as forças eletromagnéticas apenas de atração, então:

$$
\begin{aligned}
& \text { i) } F_{z} \geq 0 \text {; } \\
& \text { ii) }\left|\frac{M_{x}}{B}+\frac{M_{y}}{L}+\frac{M_{t}}{B}\right| \leq F_{z} \Leftrightarrow\left|\frac{M_{x}}{B}\right|+\left|\frac{M_{y}}{L}\right|+\left|\frac{M_{t}}{B}\right| \leq F_{z} .
\end{aligned}
$$

Escrevendo-se as equações (3.35)-(3.38) na forma matricial tem-se que:

$$
\left[\begin{array}{cccc}
1 & 1 & 1 & 1 \\
1 & 1 & -1 & -1 \\
-1 & 1 & 1 & -1 \\
1 & -1 & 1 & -1
\end{array}\right]\left[\begin{array}{l}
F_{1}(t) \\
F_{2}(t) \\
F_{3}(t) \\
F_{4}(t)
\end{array}\right]=\left[\begin{array}{c}
F_{z}(t) \\
\frac{M_{x}(t)}{B} \\
\frac{M_{y}(t)}{L} \\
\frac{M_{x}(t)}{B}
\end{array}\right] .
$$


Resolvendo o sistema tem-se que:

$$
\left[\begin{array}{l}
F_{1}(t) \\
F_{2}(t) \\
F_{3}(t) \\
F_{4}(t)
\end{array}\right]=\frac{1}{4}\left[\begin{array}{cccc}
1 & 1 & -1 & 1 \\
1 & 1 & 1 & -1 \\
1 & -1 & 1 & 1 \\
1 & -1 & -1 & -1
\end{array}\right]\left[\begin{array}{c}
F_{z}(t) \\
\frac{M_{x}(t)}{B} \\
\frac{M_{y}(t)}{L} \\
\frac{M_{x}(t)}{B}
\end{array}\right]=\frac{1}{4}\left[\begin{array}{l}
F_{z}(t)+\frac{M_{x}(t)}{B}-\frac{M_{y}(t)}{L}+\frac{M_{t}(t)}{B} \\
F_{z}(t)+\frac{M_{x}(t)}{B}+\frac{M_{y}(t)}{L}-\frac{M_{t}(t)}{B} \\
F_{z}(t)-\frac{M_{x}(t)}{B}+\frac{M_{y}(t)}{L}+\frac{M_{t}(t)}{B} \\
F_{z}(t)-\frac{M_{x}(t)}{B}-\frac{M_{y}(t)}{L}-\frac{M_{t}(t)}{B}
\end{array}\right]
$$

As forças eletromagnéticas de suspensão são obtidas diretamente da equação (3.41).

\subsubsection{Distribuição das forças para o modelo SISO}

No bloco de comando de atuadores, onde se considera o modelo SISO, as forças eletromagnéticas $F_{1}(\mathrm{t}), F_{2}(\mathrm{t}), F_{3}(\mathrm{t})$ e $F_{4}(\mathrm{t})$ são diretamente aquelas que saem dos respectivos controladores.

\subsection{Bloco de processamento de medidas}

A proposta do bloco de "Processamento de Medidas" é estimar as medidas de posição e velocidade do carro utilizando as medidas dos sensores.

\subsubsection{Processamento de medidas de posição}

O propósito deste bloco é estimar o entreferro $z(t)$ e os ângulos $\phi(t), \theta(t)$ e $\xi(t)$, em função das quatro medidas de entreferro $z_{p}(t)(p=1, \ldots, 4)$, conforme apresentado em (BITTAR,1998) e descrito a seguir. 
O movimento vertical ("heave") $z(t)$ é dado pelo valor médio da medida dos quatro entreferro $z_{p}(t)(p=1, \ldots, 4)$, ou seja:

$$
z(t)=\frac{1}{4}\left(z_{1}(t)+z_{2}(t)+z_{3}(t)+z_{4}(t)\right)
$$

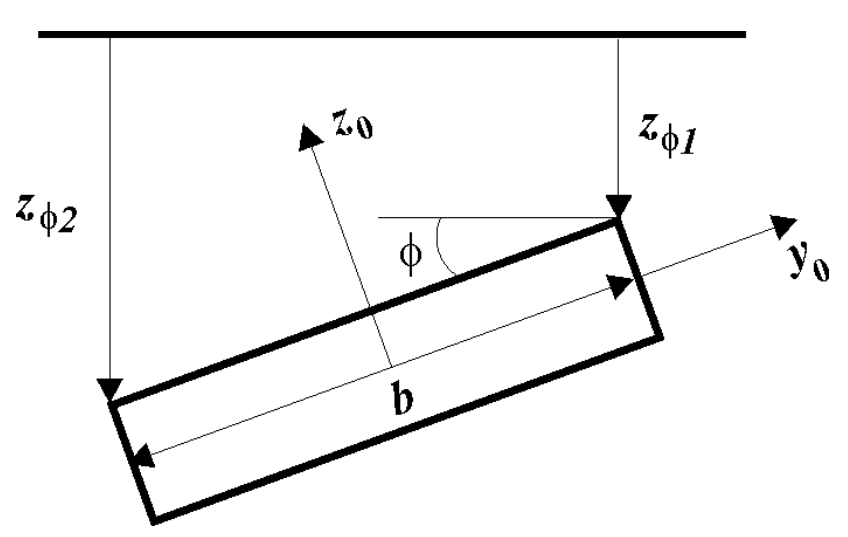

figura 3.9: seção do veículo no plano $y_{0}-z_{0}$.

No cálculo do ângulo $\phi(t)$ de rolagem ("roll”), considera-se , por exemplo, o movimento em torno do eixo $x_{0}$ (figura 3.9). Nesta figura, assumindo-se pequenos desvios dos entreferros em relação ao valor nominal e definindo:

$$
\begin{aligned}
& z_{\phi 1}(t)=\frac{z_{1}(t)+z_{2}(t)}{2}, \\
& z_{\phi 2}(t)=\frac{z_{3}(t)+z_{4}(t)}{2},
\end{aligned}
$$

o ângulo $\phi(t)$ pode ser aproximado por:

$$
\operatorname{sen} \phi(t) \cong \phi(t)=\frac{z_{\phi 2}(t)-z_{\phi 1}(t)}{b}=\frac{1}{2 b}\left(-z_{1}(t)-z_{2}(t)+z_{3}(t)+z_{4}(t)\right),
$$

onde $b(0,497 m)$ é a distância entre os sensores ao longo do eixo $y_{0}$. 


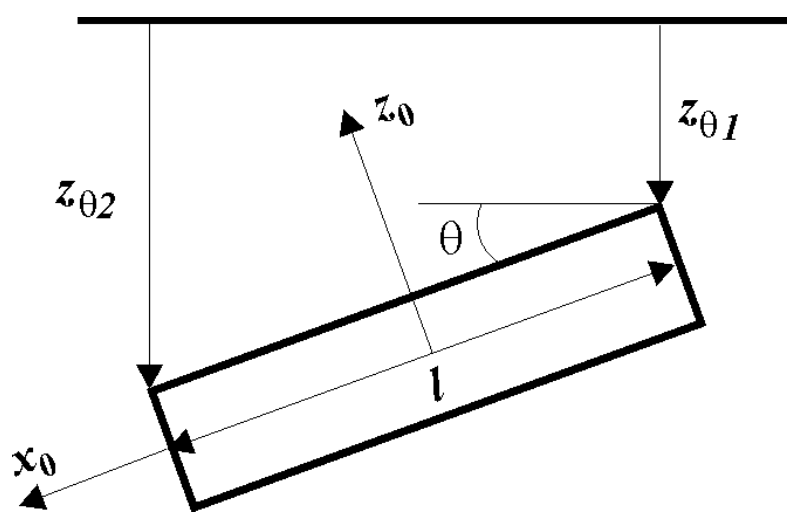

figura 3.10: seção do veículo no plano $x_{0}-z_{0}$.

No cálculo do ângulo $\theta(t)$ de arfagem ("pitch”), considera-se, por exemplo, o movimento em torno do eixo $y_{0}$ (figura 3.10). Nesta figura, assumindo-se pequenos desvios dos entreferros em relação ao valor nominal e definindo:

$$
\begin{aligned}
& z_{\theta 1}(t)=\frac{z_{2}(t)+z_{3}(t)}{2}, \\
& z_{\theta 2}(t)=\frac{z_{1}(t)+z_{4}(t)}{2},
\end{aligned}
$$

o ângulo $\theta(t)$ pode ser aproximado por:

$$
\operatorname{sen} \theta(t) \cong \theta(t)=\frac{z_{\theta 2}(t)-z_{\theta 1}(t)}{l}=\frac{1}{2 l}\left(z_{1}(t)-z_{2}(t)-z_{3}(t)+z_{4}(t)\right),
$$

onde $l(0,513 m)$ é a distância entre os sensores ao longo do eixo $x_{0}$. 

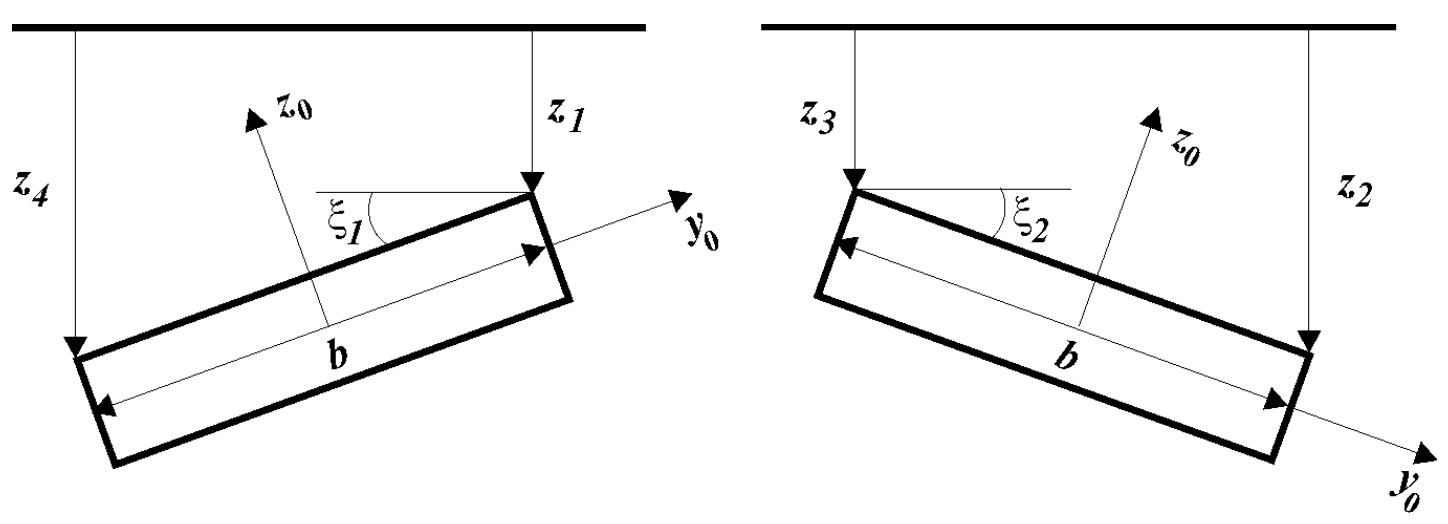

figura 3.11: Ângulos $\xi_{1}(t)$ e $\xi_{2}(t)$.

No cálculo do ângulo $\xi(t)$ de torção, considerando a figura 3.11 e definindo:

$$
\begin{aligned}
& \operatorname{sen} \xi_{1}(t) \cong \xi_{1}(t)=\frac{z_{4}(t)-z_{1}(t)}{b}, \\
& \operatorname{sen} \xi_{2}(t) \cong \xi_{2}(t)=\frac{z_{2}(t)-z_{3}(t)}{b},
\end{aligned}
$$

e supondo que:

$$
\xi(t)=\frac{\xi_{1}(t)+\xi_{2}(t)}{2}
$$

então:

$$
\xi(t)=\frac{1}{2 b}\left(-z_{1}(t)+z_{2}(t)-z_{3}(t)+z_{4}(t)\right)
$$




\subsubsection{Processamento de medidas de velocidade}

A função deste bloco é estimar a velocidade do movimento vertical $\dot{z}(t)$ e as velocidades angulares $\dot{\phi}(t), \dot{\theta}(t)$ e $\dot{\xi}(t)$, a partir das quatro velocidades estimadas $\dot{z}_{p}(t)(p=1, \ldots, 4)$. As velocidades $\dot{z}_{p}(t)(p=1, \ldots, 4)$ são calculadas, conforme definido no capítulo 2 , através das medidas de aceleração $\ddot{z}_{p}(t)(p=1, \ldots, 4)$, disponíveis em cada canto do veículo.

O procedimento é análogo ao da medida de posição, descrito anteriormente, mas agora considerando a velocidade.

A velocidade do movimento vertical ("heave") $\dot{z}(t)$ é dada pelo valor médio das quatro velocidades $\dot{z}_{p}(t)(p=1, \ldots, 4)$, ou seja:

$$
\dot{z}(t)=\frac{1}{4}\left(\dot{z}_{1}(t)+\dot{z}_{2}(t)+\dot{z}_{3}(t)+\dot{z}_{4}(t)\right) .
$$

No cálculo da velocidade angular $\dot{\phi}(t)$ de rolagem ("roll"), tem-se, derivando a equação (3.45), que:

$$
\dot{\phi}(t)=\frac{1}{2 b}\left(-\dot{z}_{1}(t)-\dot{z}_{2}(t)+\dot{z}_{3}(t)+\dot{z}_{4}(t)\right) .
$$

No cálculo da velocidade angular $\dot{\theta}(t)$ de arfagem ("pitch"), tem-se, derivando a equação (3.48), que:

$$
\dot{\theta}(t)=\frac{1}{2 l}\left(\dot{z}_{1}(t)-\dot{z}_{2}(t)-\dot{z}_{3}(t)+\dot{z}_{4}(t)\right) .
$$

No cálculo da velocidade angular $\dot{\xi}(t)$ de torção, tem-se, derivando a equação (3.52), que:

$$
\dot{\xi}(t)=\frac{1}{2 b}\left(-\dot{z}_{1}(t)+\dot{z}_{2}(t)-\dot{z}_{3}(t)+\dot{z}_{4}(t)\right) .
$$




\section{Capítulo 4}

\section{RLQ COM ALOCAÇÃO DOS PÓLOS EM MALHA FECHADA VIA LUGAR DAS RAÍZES}

Neste capítulo, no intuito de auxiliar o entendimento do projeto dos controladores, é apresentado um breve resumo teórico com os principais aspectos referentes à metodologia de projeto de controladores RLQ, que utiliza o método do lugar das raízes para definição dos pólos de malha fechada e obtenção das matrizes pesos de controle e estado.

Maiores detalhes sobre esta metodologia podem ser encontrados em (ATHANS, 1981) e (KAILATH, 1980).

\subsection{Introdução}

Considere o seguinte problema de controle ótimo baseado em índice de desempenho quadrático, dado por:

$$
\min J=\int_{0}^{\infty} x^{\prime}(t) Q x(t)+u^{\prime}(t) \rho u(t) d t
$$

sendo $Q$ uma matriz de peso para os estados do sistema e $\rho$ um parâmetro de peso para entrada de controle, sujeito às restrições:

$$
\begin{aligned}
& \dot{x}(t)=A x(t)+B u(t) \\
& y(t)=C x(t)
\end{aligned}
$$

com:

- $\quad x(t) \in \mathfrak{R}^{n}, u(t)$ escalar e $y(t)$ escalar;

- matrizes de dimensões compatíveis;

- $\quad \rho>0 ; Q=Q^{\prime}>0$;

- os pares $(A, B)$ e $\left(A, Q^{1 / 2}\right)$ controlável e observável, respectivamente. 
Deseja-se encontrar uma lei de controle ótimo, denominada de realimentação de estados, onde o índice de desempenho ou funcional (4.1) é minimizado. A solução para este problema é dada por:

$$
u(t)=-K x(t)
$$

onde $K$ é chamada de matriz de ganho da realimentação de estados e dada por:

$$
K=\frac{1}{\rho} B^{\prime} P
$$

e P é a solução simétrica definida positiva da Equação Algébrica de Riccati (EAR):

$$
P A+A^{\prime} P-\frac{1}{\rho} P B B^{\prime} P+Q=0
$$

\subsection{Interpretação}

Quando se lida com projeto de RLQ, é muito importante entendermos que a noção do que é considerada uma "saída" é uma questão de interpretação. Definindose o escalar $\hat{y}(t)$ como:

$$
\hat{y}(t)=\hat{C} x(t)
$$

onde $\hat{C}$ é uma matriz parâmetro de projeto, pode-se pensar a partir da equação (4.2) no seguinte sistema SISO:

$$
\begin{aligned}
\dot{x}(t) & =A x(t)+B u(t) \\
\hat{y}(t) & =\hat{C} x(t)
\end{aligned}
$$

No contexto de projeto do RLQ, como decisão de projeto, se escolhermos a matriz de peso do estado como sendo:

$$
Q=\hat{C}^{\prime} \hat{C},
$$


o índice de desempenho é modificado e dado em termos do vetor de saída $\hat{y}(t)$ ao invés do vetor de estado. Isto é:

$$
J=\int_{0}^{\infty} \hat{y}^{\prime}(t) \hat{y}(t)+u^{\prime}(t) \rho u(t) d t
$$

de modo que a "saída" $\hat{y}(t)$ é uma função escalar que será regulada em zero.

A figura 4.1 apresenta do diagrama de blocos para o RLQ.

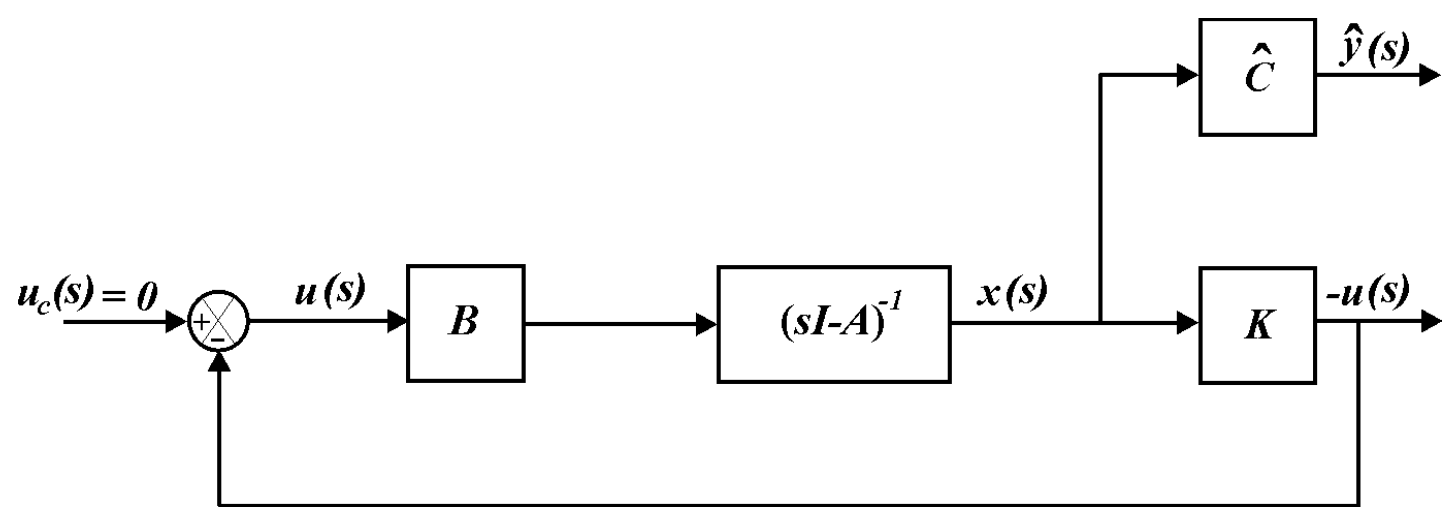

Figura 4.1: Diagrama de blocos do RLQ.

\subsection{Identidade de Kalman}

Considerando a figura 4.1, as seguintes funções de transferência podem ser definidas:

$$
G_{O L}(s)=\hat{C}(s I-A)^{-1} B
$$

e

$$
G_{L Q}(s)=K(s I-A)^{-1} B
$$

Conforme apresentado em (CRUZ,1996) e (ATHANS,1981), a identidade de Kalman é uma expressão que relaciona as equações (4.10) e (4.11), que são funções de transferência escalar, da seguinte maneira:

$$
\left(1+G_{L Q}(-s)\right)\left(1+G_{L Q}(s)\right)=1+\frac{1}{\rho} G_{O L}(-s) G_{O L}(s) .
$$




\subsection{O Lugar das Raízes}

Definindo $a(s)$ como o polinômio característico de malha aberta, associado a $G_{O L}(s)$, isto é:

$$
a(s)=\operatorname{det}(s I-A)
$$

então as raízes de $a(s)$ são os pólos de malha aberta.

Definindo o polinômio b(s) como segue:

$$
b(s)=a(s) G_{O L}(s)=a(s) \hat{C}(s I-A)^{-1} B,
$$

então as raízes de $b(s)$ são os "zeros auxiliares"1 de malha aberta.

Multiplicando-se ambos os lados da identidade de Kalman por $a(-s) a(s)$, obtém-se:

$$
a(-s)\left(1+G_{L Q}(-s)\right)\left(1+G_{L Q}(s)\right) a(s)=a(-s) a(s)+\frac{1}{\rho} b(-s) b(s) .
$$

Da análise da figura 4.1, tem-se que a função de transferência em malha fechada do RLQ é:

$$
\frac{G_{L Q}(s)}{1+G_{L Q}(s)} .
$$

Deste modo os pólos de malha fechada serão as raízes de $\left(1+G_{L Q}(s)\right)$, que são os autovalores da matriz $[A-B K]$ do sistema em malha fechada.

Se $s$ é um pólo de malha fechada, então a diferença de retorno $\left(1+G_{L Q}(s)\right)=0$. Portanto, olhando para a equação (4.15), os pólos de malha fechada do RLQ devem ser as raízes da equação:

\footnotetext{
${ }^{1}$ Estes zeros são chamados de auxiliares, pois dependem da matriz $\hat{C}$ de projeto de não da matriz $C$ do sistema.
} 


$$
a(-s) a(s)+\frac{1}{\rho} b(-s) b(s)=0
$$

A equação (4.17) está na forma padrão para análise do lugar das raízes.

Para uma melhor visualização, a figura 4.2 representa o diagrama de blocos para o lugar das raízes da equação (4.17). Os "zeros auxiliares" de malha aberta são as raízes de $b(-s) b(s)$, enquanto os pólos de malha aberta são raízes de $a(-s) a(s)$. O escalar $\frac{1}{\rho}$ desempenha o papel do "ganho" para o traçado do lugar das raízes.

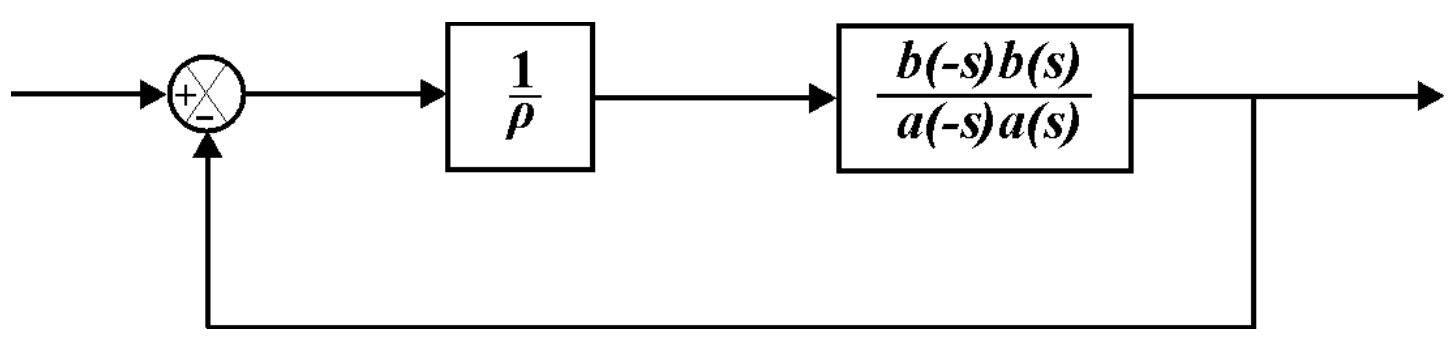

Figura 4.2: Diagrama de blocos para o Lugar das Raízes

Conclui-se que os pólos de malha fechada do sistema dependem da matriz de peso do estado $(Q)$ e do escalar peso do controle $(\rho)$. Os efeitos das variações destes parâmetros de projeto podem ser visualizados através da técnica do lugar das raízes, que mostra o movimento dos pólos de malha fechada quando o peso do controle $(\rho)$ varia.

\subsection{Procedimento}

A seguir está apresentado o procedimento para escolha dos pólos de malha fechada a partir da escolha da matriz $Q$ e do parâmetro $\rho$.

Antes de iniciar o procedimento, sabe-se que a função de transferência dada pela equação (4.10), ou seja, 


$$
G_{O L}(s)=\hat{C}(s I-A)^{-1} B=\frac{b(s)}{a(s)},
$$

têm $n$ pólos (raízes de $a(s)$ ) e $m$ "zeros auxiliares" (raízes de $b(s)$ ). As matrizes $A$ e $B$ fazem parte do modelo nominal, logo são restrições, e a matriz de projeto $(\hat{C})$ será escolhida convenientemente.

Passo 1: deve-se escolher a matriz $\hat{C}$, de projeto, que influenciará diretamente na localização dos $m$ "zeros auxiliares" de malha aberta de $G_{O L}(s)$.

Passo 2: posicionam-se no plano $s$ os $m$ "zeros auxiliares" de malha aberta de $G_{O L}(s)$, isto é, as raízes de $b(s)$.

Passo 3: posicionam-se no plano $s$ os $n$ pólos de malha aberta de $G_{O L}(s)$, isto é, as raízes de $a(s)$. Estes pólos dependem unicamente da matriz $A$.

Passo 4: Os "zeros auxiliares" e pólos de malha aberta de $G_{O L}(-s)$, respectivamente, raízes de $b(-s)$ e $a(-s)$, são definidos e posicionados no plano $s$ como imagens, em relação ao eixo $j w$, dos zeros $(m)$ e pólos $(n)$ definidos nos passos 2 e 3 , respectivamente. Têm-se, agora, no plano $s$, todos os "zeros auxiliares" de malha aberta $(2 m)$ e todos os pólos de malha aberta $(2 n)$.

Passo 5: esboça-se, em função do parâmetro $\rho$, o lugar das raízes. O lugar das raízes será completamente simétrico em relação ao eixo $j w$. Neste caso como o RLQ é sempre estável, utiliza-se apenas a parte do lugar das raízes localizada no semiplano esquerdo do plano $s$.

Passo 6: definido os pólos de malha fechada que atendem as especificações de projeto, tem-se o valor do parâmetro $\rho$.

Passo 7: definido os pólos de malha fechada que satisfazem as condições de projeto e tendo-se a matriz $\hat{C}$, calcula-se, pela equação (4.8), o valor de $Q$.

Passo 8: definida a matriz $\mathrm{Q}$ e o parâmetro $\rho$, e tendo-se as matrizes $A$ e $B$, calcula-se a matriz ganho de realimentação de estados $(K)$, através da função "lqr", disponível no software MATLAB, onde $K=\operatorname{lqr}(A, B, Q, \rho)$. 


\section{Capítulo 5}

\section{PROJETO DOS CONTROLADORES}

Neste capítulo são apresentados: as especificações de projeto, as análises em regime estacionário, os projetos e as simulações obtidas com a implementação do controlador ótimo do tipo RLQ. Este controlador foi implementado em cada um dos movimentos (vertical, rolagem, arfagem e torção). Uma nova abordagem foi adotada na escolha da matriz peso dos estados e do parâmetro de controle, com a utilização do método do lugar das raízes para verificação do comportamento dos pólos de malha fechada.

Está também abordado o controlador $\mathrm{H}_{2} / \mathrm{H}_{\infty}$ para o modelo de corpo rígido com realimentação da saída.

\subsection{Especificações de projeto}

Como condições de projeto foi definido, para uma entrada em degrau, que:

- o erro de regime estacionário seja nulo;

- o sobre-sinal seja menor que $40 \%$;

- o tempo de subida seja o menor possível, mas sem saturar o atuador, ou seja, que a tensão $v_{i}(t)$, dada pela equação (3.10), não ultrapasse o valor de $5 \mathrm{~V}$;

- o degrau para o movimento vertical será o deslocamento de 7,5mm (condição de repouso, com o veículo apoiado sobre a via) para 5,0 $\mathrm{mm}$;

- o degrau para o movimento de rolagem, arfagem e torção será o deslocamento de -0,008 rad para 0 rad, estando o veículo em levitação no valor de referência $(5 \mathrm{~mm})$.

Mas o principal objetivo neste projeto é que o sistema se mantenha estável mesmo quando na presença de uma perturbação, ou seja, o desvio do valor do entreferro e o tempo de acomodação sejam o menor possível. 
Como critério de desempenho em relação à perturbação foi definido que:

- o tempo de acomodação seja menor que $0,8 s$;

- o desvio do valor do entreferro seja o menor possível, mas respeitando-se a condição de sobre-sinal definida para a entrada em degrau.

As perturbações aplicadas são:

- degrau de $10 \mathrm{~kg}$ no movimento vertical;

- degrau de $25 \mathrm{Nm}$ nos movimentos de rolagem, arfagem e torção;

- degrau de $2,5 \mathrm{~kg}$ no movimento vertical do projeto modelo SISO.

\subsection{Sistema na forma de equações de estado}

As equações do modelo do sistema, obtidas no capítulo 3, foram transformadas para a forma de equações de estado, conforme apresentado nos itens seguintes.

\subsubsection{Movimento vertical com controlador}

$\mathrm{Na}$ figura 5.1 tem-se o diagrama de blocos do movimento vertical com o respectivo controlador.

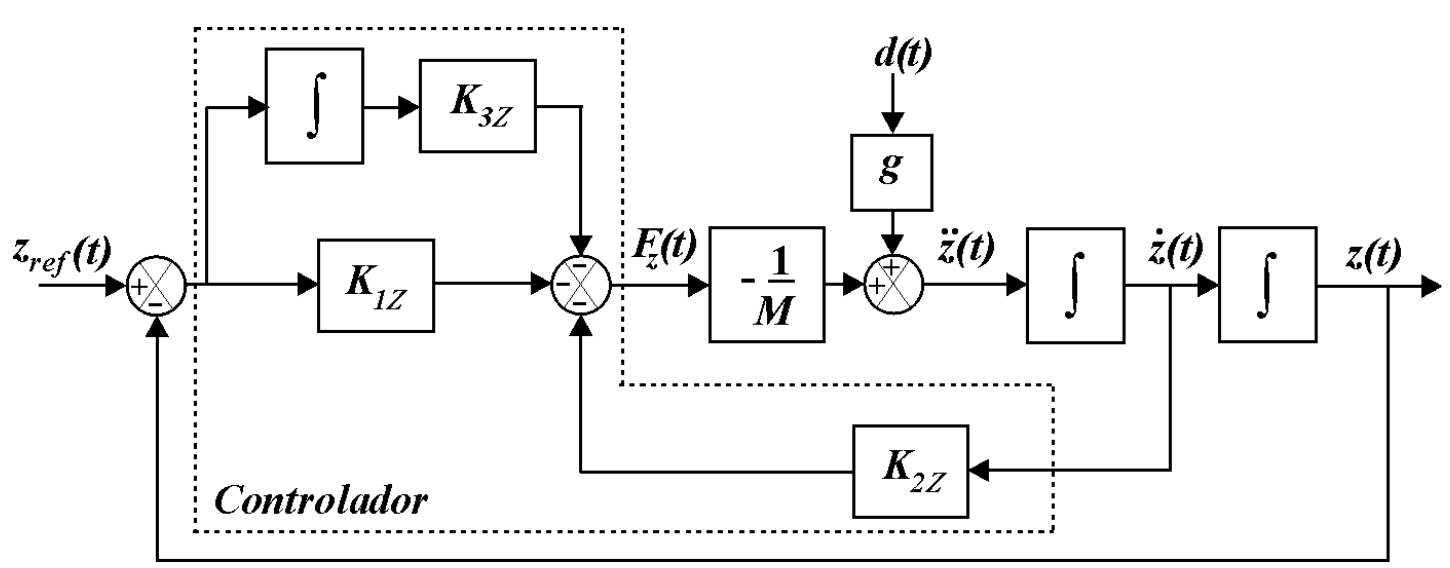

Figura 5.1: Diagrama de blocos do movimento vertical com o controlador. 
Considerando as equações (3.1) ou (3.4), tem-se que:

$$
M \ddot{z}(t)=\operatorname{Mgd}(t)-F_{z}(t) \Rightarrow \ddot{z}(t)=g d(t)-\frac{1}{M} F_{z}(t),
$$

onde $d(t)$ e $z_{r e f}(t)$ são degraus, respectivamente, unitário e de amplitude $z_{r e f}$.

Definindo as variáveis de estado: $\left\{\begin{array}{l}x_{1}(t)=z_{r e f}(t)-z(t) \\ x_{2}(t)=\dot{z}(t) \\ x_{3}(t)=\int x_{1}(t) d t\end{array}\right.$

e considerando $F_{\mathrm{z}}(t)=u(t)$, as seguintes equações de estado e saída são obtidas para $t>0$ :

$$
\left\{\begin{array}{l}
\dot{x}_{1}(t)=-\dot{z}(t)=-x_{2}(t), \\
\dot{x}_{2}(t)=\ddot{z}(t)=-\frac{1}{M} F_{z}(t)+g d(t)=-\frac{1}{M} u(t)+g d(t), \\
\dot{x}_{3}(t)=x_{1}(t) \\
y(t)=z(t)=-x_{1}(t)+z_{r e f} .
\end{array}\right.
$$

$\mathrm{Na}$ forma matricial resulta o seguinte modelo de estados:

$$
\begin{aligned}
& \dot{x}(t)=\left[\begin{array}{ccc}
0 & -1 & 0 \\
0 & 0 & 0 \\
1 & 0 & 0
\end{array}\right] x(t)+\left[\begin{array}{c}
0 \\
-1 / M \\
0
\end{array}\right] u(t)+\left[\begin{array}{l}
0 \\
g \\
0
\end{array}\right] d(t), \\
& y(t)=\left[\begin{array}{lll}
-1 & 0 & 0
\end{array}\right] x(t)+z_{r e f},
\end{aligned}
$$

ou simplificadamente:

$$
\begin{aligned}
& \dot{x}(t)=A x(t)+B u(t)+D_{v} d(t), \\
& y(t)=C x(t)+z_{\text {ref }},
\end{aligned}
$$

com:

$$
A=\left[\begin{array}{ccc}
0 & -1 & 0 \\
0 & 0 & 0 \\
1 & 0 & 0
\end{array}\right], \quad B=\left[\begin{array}{c}
0 \\
-1 / M \\
0
\end{array}\right], \quad C=\left[\begin{array}{lll}
-1 & 0 & 0
\end{array}\right], \quad D_{v}=\left[\begin{array}{l}
0 \\
g \\
0
\end{array}\right]
$$


O integrador foi introduzido no controlador para eliminar o efeito em regime permanente da perturbação constante devido à gravidade $(g)$.

\subsubsection{Movimento de rolagem com controlador}

A figura 5.2 apresenta o diagrama de blocos do movimento de rolagem com o respectivo controlador.

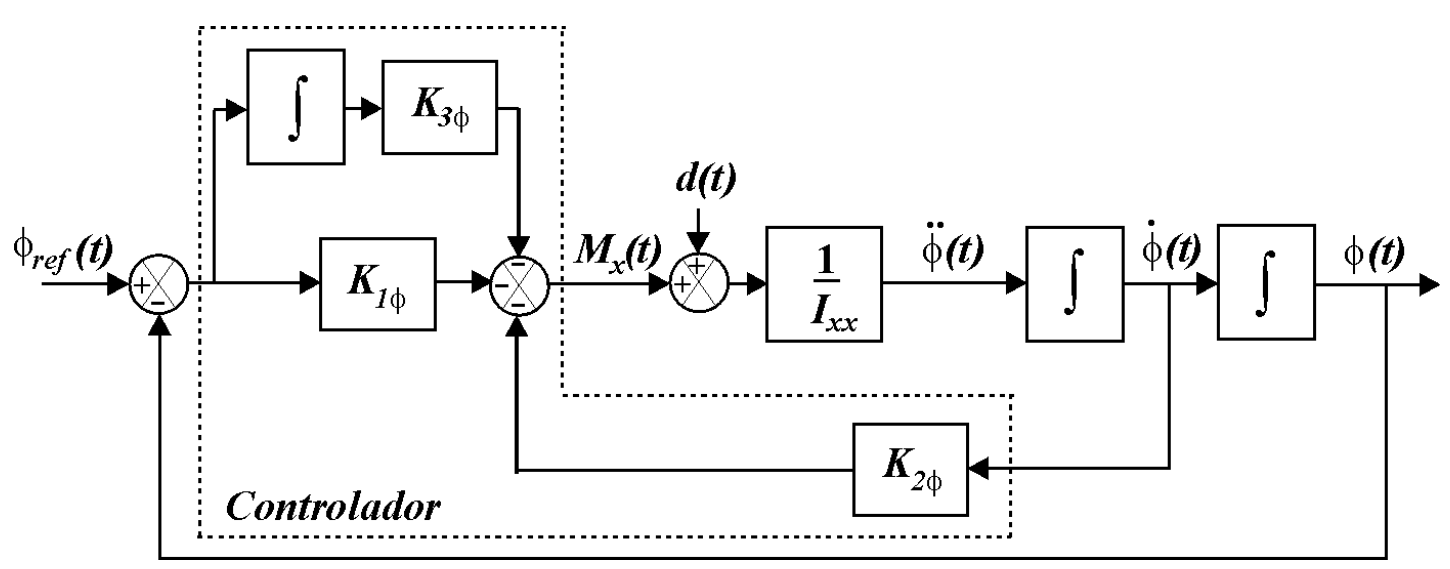

Figura 5.2: Diagrama de blocos do movimento de rolagem com o controlador.

Considerando as equações (3.2) ou (3.5) e a existência de uma perturbação $(d(t))$ na entrada da planta, tem-se que:

$$
I_{x x} \ddot{\phi}(t)=M_{x}(t)+d(t),
$$

onde $\phi_{r e f}(t)$ é um degrau de valor $\phi_{r e f}$.

Definindo as variáveis de estado: $\left\{\begin{array}{l}x_{1}(t)=\phi_{r e f}(t)-\phi(t), \\ x_{2}(t)=\dot{\phi}(t), \\ x_{3}(t)=\int x_{1}(t) d t,\end{array}\right.$

e considerando $M_{\mathrm{x}}(t)=u(t)$, as seguintes equações de estado e saída são obtidas para $t>0$ : 


$$
\left\{\begin{array}{l}
\dot{x}_{1}(t)=-\dot{\phi}(t)=-x_{2}(t) \\
\dot{x}_{2}(t)=\ddot{\phi}(t)=\frac{1}{I_{x x}}\left(M_{x}(t)+d(t)\right)=\frac{1}{I_{x x}} u(t)+\frac{1}{I_{x x}} d(t), \\
\dot{x}_{3}(t)=x_{1}(t) \\
y(t)=\phi(t)=-x_{1}(t)+\phi_{r e f} .
\end{array}\right.
$$

$\mathrm{Na}$ forma matricial resulta o seguinte modelo de estados:

$$
\begin{aligned}
\dot{x}(t) & =\left[\begin{array}{ccc}
0 & -1 & 0 \\
0 & 0 & 0 \\
1 & 0 & 0
\end{array}\right] x(t)+\left[\begin{array}{c}
0 \\
1 / I_{x x} \\
0
\end{array}\right] u(t)+\left[\begin{array}{c}
0 \\
1 / I_{x x} \\
0
\end{array}\right] d(t), \\
y(t) & =\left[\begin{array}{lll}
-1 & 0 & 0
\end{array}\right] x(t)+\phi_{r e f},
\end{aligned}
$$

ou simplificadamente:

$$
\begin{aligned}
& \dot{x}(t)=A x(t)+B u(t)+D_{r}(t), \\
& y(t)=C x(t)+\phi_{r e f},
\end{aligned}
$$

com:

$$
A=\left[\begin{array}{ccc}
0 & -1 & 0 \\
0 & 0 & 0 \\
1 & 0 & 0
\end{array}\right], \quad B=\left[\begin{array}{c}
0 \\
1 / I_{x x} \\
0
\end{array}\right], \quad C=\left[\begin{array}{lll}
-1 & 0 & 0
\end{array}\right], \quad D_{r}=\left[\begin{array}{c}
0 \\
1 / I_{x x} \\
0
\end{array}\right]
$$

O integrador foi introduzido no controlador para eliminar o efeito em regime estacionário de uma perturbação $d(t)$ na entrada da planta ou um desbalanceamento da massa do veículo entre os quatro cantos.

\subsubsection{Movimento de arfagem com controlador}

A figura 5.3 apresenta o diagrama de blocos do movimento de arfagem com o respectivo controlador. 


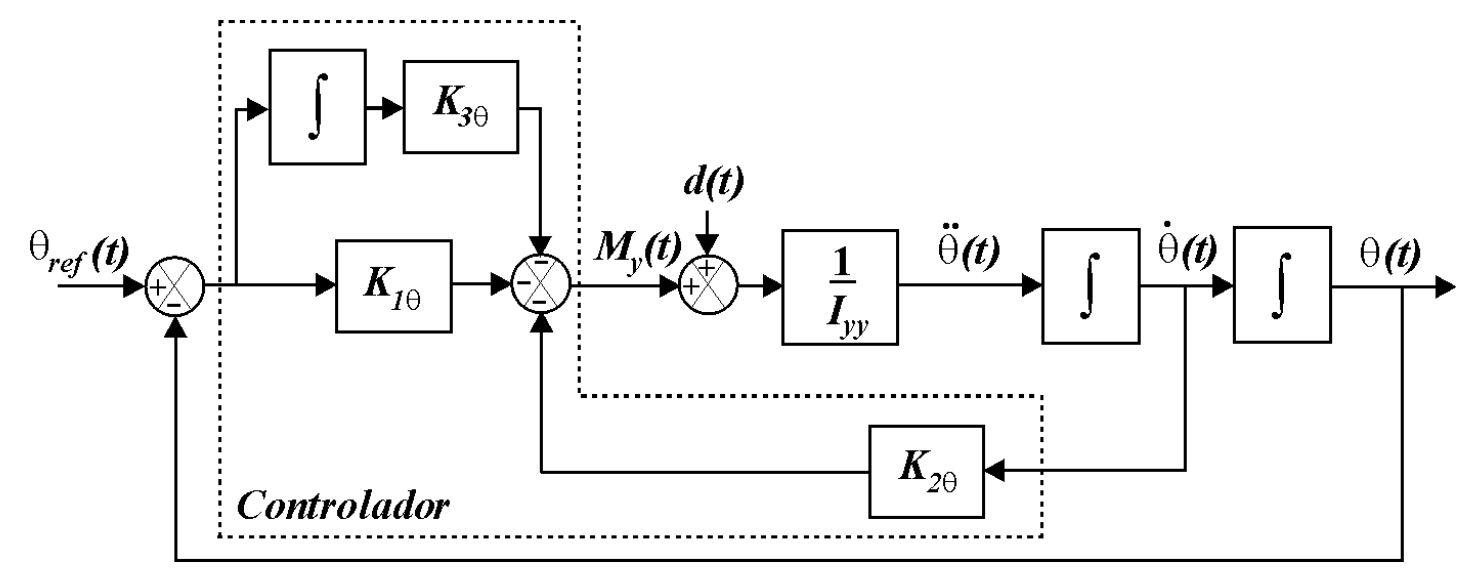

Figura 5.3: Diagrama de blocos do movimento de arfagem com o controlador.

Considerando as equações (3.3) ou (3.6) e a existência de uma perturbação $(d(t))$ na entrada da planta, tem-se que:

$$
I_{y y} \ddot{\theta}(t)=M_{y}(t)+d(t),
$$

onde $\theta_{\text {ref }}(t)$ é um degrau de valor $\theta_{r e f}$.

Definindo as variáveis de estado: $\left\{\begin{array}{l}x_{1}(t)=\theta_{r e f}(t)-\theta(t), \\ x_{2}(t)=\dot{\theta}(t), \\ x_{3}(t)=\int x_{1}(t) d t,\end{array}\right.$

e considerando $M_{\mathrm{y}}(t)=u(t)$, as seguintes equações de estado e saída são obtidas para $t>0$ :

$$
\left\{\begin{array}{l}
\dot{x}_{1}(t)=-\dot{\theta}(t)=-x_{2}(t), \\
\dot{x}_{2}(t)=\ddot{\theta}(t)=\frac{1}{I_{y y}}\left(M_{y}(t)+d(t)\right)=\frac{1}{I_{y y}} u(t)+\frac{1}{I_{y y}} d(t), \\
y(t)=\theta(t)=-x_{1}(t)+\theta_{r e f} .
\end{array}\right.
$$

$\mathrm{Na}$ forma matricial resulta o seguinte modelo de estados: 


$$
\begin{aligned}
& \dot{x}(t)=\left[\begin{array}{ccc}
0 & -1 & 0 \\
0 & 0 & 0 \\
1 & 0 & 0
\end{array}\right] x(t)+\left[\begin{array}{c}
0 \\
1 / I_{y y} \\
0
\end{array}\right] u(t)+\left[\begin{array}{c}
0 \\
1 / I_{y y} \\
0
\end{array}\right] d(t), \\
& y(t)=\left[\begin{array}{lll}
-1 & 0 & 0
\end{array}\right] x(t)+\theta_{r e f},
\end{aligned}
$$

ou simplificadamente:

$$
\begin{aligned}
& \dot{x}(t)=A x(t)+B u(t)+D_{a} d(t), \\
& y(t)=C x(t)+\theta_{r e f}
\end{aligned}
$$

com:

$$
A=\left[\begin{array}{ccc}
0 & -1 & 0 \\
0 & 0 & 0 \\
1 & 0 & 0
\end{array}\right], \quad B=\left[\begin{array}{c}
0 \\
1 / I_{y y} \\
0
\end{array}\right], \quad C=\left[\begin{array}{lll}
-1 & 0 & 0
\end{array}\right], \quad D_{a}=\left[\begin{array}{c}
0 \\
1 / I_{y y} \\
0
\end{array}\right]
$$

\subsubsection{Movimento de torção com controlador}

A figura 5.4 apresenta o diagrama de blocos do movimento de torção com o respectivo controlador.

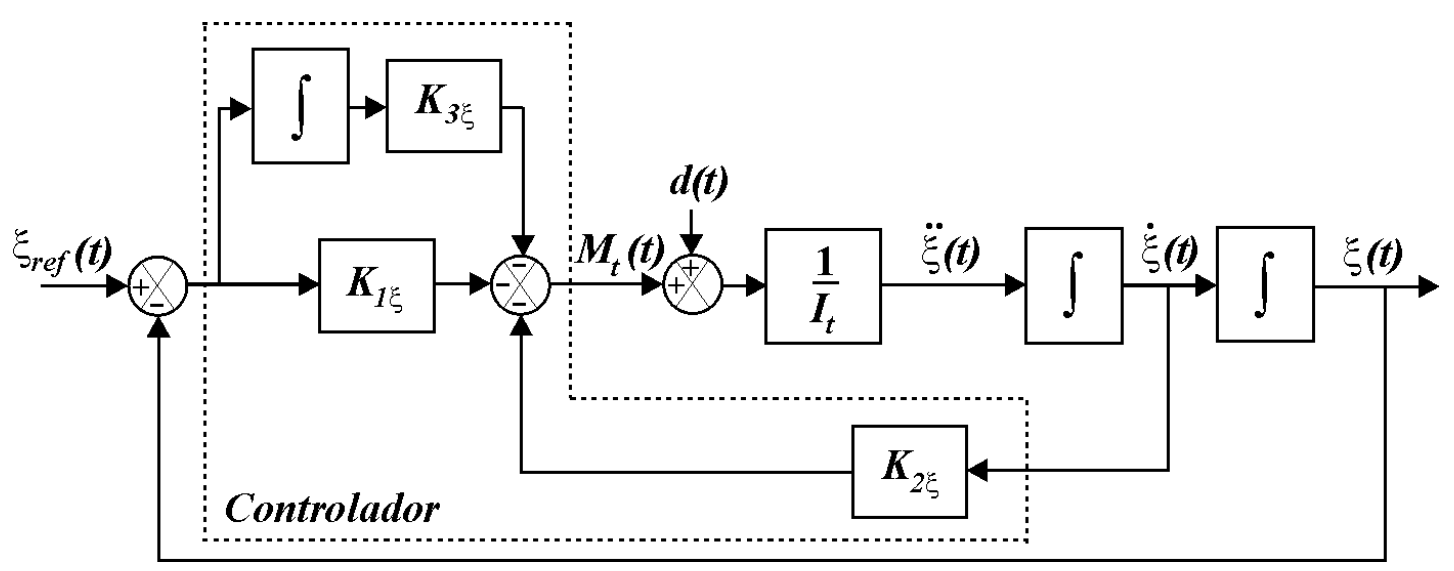

Figura 5.4: Diagrama de blocos do movimento de torção com o controlador. 
Considerando a equação (3.7) e a existência de uma perturbação $(d(t))$ na entrada da planta, tem-se que:

$$
I_{t} \ddot{\xi}(t)=M_{t}(t)+d(t),
$$

onde $\xi_{\text {ref }}(t)$ é um degrau de valor $\xi_{\text {ref }}$.

Definindo as variáveis de estado: $\left\{\begin{array}{l}x_{1}(t)=\xi_{r e f}(t)-\xi(t), \\ x_{2}(t)=\dot{\xi}(t), \\ x_{3}(t)=\int x_{1}(t) d t\end{array}\right.$

e considerando $M_{\mathrm{t}}(t)=u(t)$, as seguintes equações de estado e saída são obtidas para $t>0$ :

$$
\left\{\begin{array}{l}
\dot{x}_{1}(t)=-\dot{\xi}(t)=-x_{2}(t), \\
\dot{x}_{2}(t)=\ddot{\xi}(t)=\frac{1}{I_{t}}\left(M_{t}(t)+d(t)\right)=\frac{1}{I_{t}} u(t)+\frac{1}{I_{t}} d(t), \\
y(t)=\xi(t)=-x_{1}(t)+\xi_{\text {ref }} .
\end{array}\right.
$$

$\mathrm{Na}$ forma matricial resulta o seguinte modelo de estados:

$$
\begin{aligned}
& \dot{x}(t)=\left[\begin{array}{ccc}
0 & -1 & 0 \\
0 & 0 & 0 \\
1 & 0 & 0
\end{array}\right] x(t)+\left[\begin{array}{c}
0 \\
1 / I_{t} \\
0
\end{array}\right] u(t)+\left[\begin{array}{c}
0 \\
1 / I_{t} \\
0
\end{array}\right] d(t), \\
& y(t)=\left[\begin{array}{lll}
-1 & 0 & 0
\end{array}\right] x(t)+\xi_{\text {ref }},
\end{aligned}
$$

ou simplificadamente:

$$
\begin{aligned}
& \dot{x}(t)=A x(t)+B u(t)+D_{t} d(t), \\
& y(t)=C x(t)+\xi_{\text {ref }},
\end{aligned}
$$

com: 


$$
A=\left[\begin{array}{ccc}
0 & -1 & 0 \\
0 & 0 & 0 \\
1 & 0 & 0
\end{array}\right], \quad B=\left[\begin{array}{c}
0 \\
1 / I_{t} \\
0
\end{array}\right], \quad C=\left[\begin{array}{lll}
-1 & 0 & 0
\end{array}\right], \quad D_{t}=\left[\begin{array}{c}
0 \\
1 / I_{t} \\
0
\end{array}\right]
$$

\subsection{Análise do sistema em regime estacionário}

Inicialmente foi feita uma análise da resposta do sistema em regime estacionário devido a um degrau unitário.

Para o movimento vertical pensou-se em um controlador, conforme apresentado na figura 5.1, mas sem a parte do integrador e do ganho $K_{3 Z}$. Deste modo, a função de transferência que relaciona a saída $(Z(s))$ com a entrada $\left(Z_{r e f}(s)\right)$, considerando a perturbação devido à gravidade $(D(\mathrm{~s}))$ igual a zero, é dada por:

$$
\frac{Z(s)}{Z_{r e f}(s)}=\frac{K_{1 Z}}{M s^{2}-K_{2 Z} s+K_{1 Z}}
$$

O valor em regime estacionário da saída, para uma entrada em degrau unitário é obtido pela aplicação do teorema do valor final como segue:

$$
z(\infty)=\lim _{t \rightarrow \infty} z(t)=\lim _{s \rightarrow 0} s Z(s)=s \frac{K_{1 Z}}{M s^{2}-K_{2 Z} s+K_{1 Z}} \frac{1}{s}=1
$$

Considerando nula a entrada de referência $\left(Z_{r e f}(s)=0\right)$, a função de transferência entre a saída e o distúrbio, devido a gravidade, é dada por:

$$
\frac{Z(s)}{D(s)}=\frac{M g}{M s^{2}-K_{2 Z} s+K_{1 Z}}
$$

O valor em regime estacionário da saída, devido a um distúrbio da aceleração da gravidade em degrau de valor unitário, é dado por: 


$$
z(\infty)=\lim _{s \rightarrow 0} s Z(s)=s \frac{M g}{M s^{2}-K_{2 Z} s+K_{1 Z}} \frac{1}{s}=\frac{M g}{K_{1 Z}} .
$$

Verifica-se que este controlador não consegue eliminar o erro em regime estacionário devido à aceleração da gravidade. Um integrador foi acrescentado ao controlador, figura 5.1, e o erro em regime estacionário foi eliminado.

A nova função de transferência que relaciona a saída $(Z(s))$ com a entrada $\left(Z_{r e f}(s)\right)$, considerando a perturbação devido à gravidade $(D(\mathrm{~s}))$ igual a zero, é dada por:

$$
\frac{Z(s)}{Z_{r e f}(s)}=\frac{K_{1 Z} s+K_{3 Z}}{M s^{3}-K_{2 Z} s^{2}+K_{1 Z} s+K_{3 Z}},
$$

e o valor em regime estacionário da saída é:

$$
z(\infty)=\lim _{s \rightarrow 0} s Z(s)=s \frac{K_{1 Z} s+K_{3 Z}}{M s^{3}-K_{2 Z} s^{2}+K_{1 Z} s+K_{3 Z}} \frac{1}{s}=1 .
$$

Considerando nula a entrada de referência $\left(Z_{r e f}(s)=0\right)$, a nova função de transferência entre a saída e o distúrbio, devido a gravidade, é dada por:

$$
\frac{Z(s)}{D(s)}=\frac{M g s}{M s^{3}-K_{2 Z} s^{2}+K_{1 Z} s+K_{3 Z}}
$$

e o valor em regime estacionário da saída é:

$$
z(\infty)=\lim _{s \rightarrow 0} s Z(s)=s \frac{M g s}{M s^{3}-K_{2 Z} s^{2}+K_{1 Z} s+K_{3 Z}} \frac{1}{s}=0 .
$$

Deste modo, com a inclusão do integrador, o erro em regime estacionário é nulo. Mas o sistema em malha fechada, equação (5.13), apresenta um zero de valor $-K_{3 Z} / K_{1 Z}$, que provoca um sobre-sinal maior na resposta transitória.

No movimento de rolagem, para eliminar o efeito da perturbação foi incluído também um integrador. Deste modo a função de transferência que relaciona a saída 
$(\phi(s))$ com a entrada $\left(\phi_{\text {ref }}(s)\right)$, considerando a perturbação de entrada $(D(\mathrm{~s}))$ igual a zero, é dada por:

$$
\frac{\phi(s)}{\phi_{r e f}(s)}=\frac{-\left(K_{1 \phi} s+K_{3 \phi}\right)}{I_{x x} s^{3}+K_{2 \phi} s^{2}-K_{1 \phi} s-K_{3 \phi}},
$$

e o valor em regime estacionário da saída, para uma entrada em degrau unitário é:

$$
\phi(\infty)=\lim _{s \rightarrow 0} s \phi(s)=\lim _{s \rightarrow 0} s \frac{-\left(K_{1 \phi} s+K_{3 \phi}\right)}{I_{x x} s^{3}+K_{2 \phi} s^{2}-K_{1 \phi} s-K_{3 \phi}} \frac{1}{s}=1
$$

Considerando nula a entrada de referência $\left(\phi_{r e f}(s)=0\right)$, a função de transferência entre a saída e a perturbação de entrada é dada por:

$$
\frac{\phi(s)}{D(s)}=\frac{s}{I_{x x} s^{3}+K_{2 \phi} s^{2}-K_{1 \phi} s-K_{3 \phi}}
$$

e o valor em regime estacionário da saída é:

$$
\phi(\infty)=\lim _{s \rightarrow 0} s \phi(s)=\lim _{s \rightarrow 0} s \frac{s}{I_{x x} s^{3}+K_{2 \phi} s^{2}-K_{1 \phi} s-K_{3 \phi}} \frac{1}{s}=0
$$

Os movimentos de arfagem e torção, devido às similaridades com o movimento de rolagem, apresentam erro nulo em regime estacionário para entrada em degrau.

\subsection{Controlador RLQ para o modelo de corpo rígido}

Considerando o modelo de corpo rígido, o sistema de equações é formado pelos movimentos vertical, de rolagem e de arfagem. Sendo assim, foi projetado um controlador para cada movimento. A figura 5.5 mostra o diagrama de blocos do sistema em malha fechada, com um controlador independente para cada grau de liberdade.

Os controladores foram projetados conforme o procedimento apresentado no capítulo 4. 


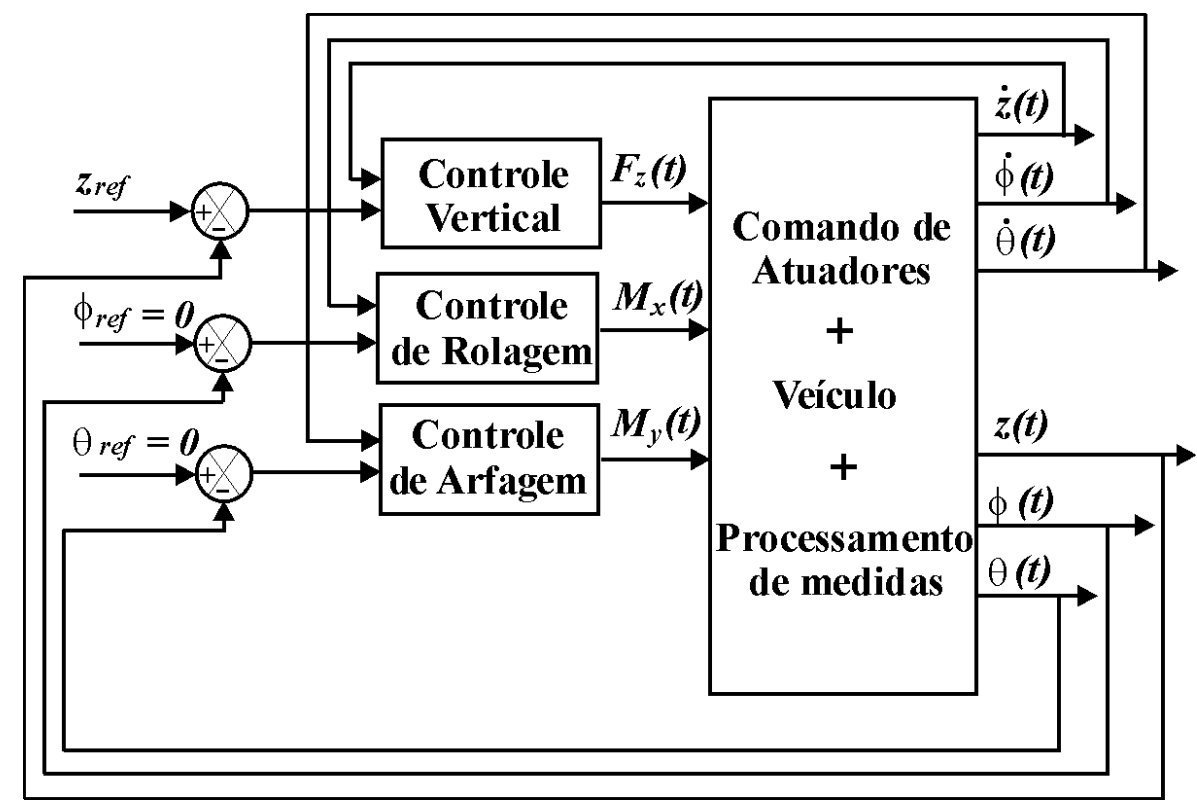

Figura 5.5: Sistema em malha fechada - modelo de corpo rígido.

\subsubsection{Controlador do movimento vertical}

Inicialmente escolhe-se a matriz de projeto $(\hat{C})$, como sendo:

$$
\hat{C}=\left[\begin{array}{lll}
\hat{c}_{1} & \hat{c}_{2} & \hat{c}_{3}
\end{array}\right]
$$

Calcula-se a função de transferência $G_{O L}(s)$ e $G_{O L}(-s)$, ou seja:

$$
G_{O L}(s)=\frac{b(s)}{a(s)}=\hat{C}(s I-A)^{-1} B=\frac{-\hat{c}_{2}\left(s^{2}-\frac{\hat{c}_{1}}{\hat{c}_{2}} s-\frac{\hat{c}_{3}}{\hat{c}_{2}}\right)}{M s^{3}}
$$

e

$$
G_{O L}(-s)=\frac{b(-s)}{a(-s)}=\hat{C}(-s I-A)^{-1} B=\frac{\hat{c}_{2}\left(s^{2}+\frac{\hat{c}_{1}}{\hat{c}_{2}} s-\frac{\hat{c}_{3}}{\hat{c}_{2}}\right)}{M s^{3}}
$$

Reescrevendo na forma de pólos e zeros, tem-se que: 


$$
\frac{b(-s) b(s)}{a(-s) a(s)}=-\left(\frac{\hat{c}_{2}}{M}\right)^{2} \frac{\left(s-\hat{z}_{1}\right)\left(s-\hat{z}_{2}\right)\left(s-\hat{z}_{3}\right)\left(s-\hat{z}_{4}\right)}{s^{6}}
$$

onde:

$$
\hat{z}_{1,2}=\frac{\hat{c}_{1} \pm \sqrt{\hat{c}_{1}^{2}+4 \hat{c}_{2} \hat{c}_{3}}}{2 \hat{c}_{2}} \text { e } \hat{z}_{3,4}=\frac{-\hat{c}_{1} \pm \sqrt{\hat{c}_{1}^{2}+4 \hat{c}_{2} \hat{c}_{3}}}{2 \hat{c}_{2}}
$$

Visto que estes "zeros auxiliares" $\left(\hat{z}_{1}, \hat{z}_{2}, \hat{z}_{3} e \hat{z}_{4}\right)$ de malha aberta são influenciados diretamente pelos valores $\hat{c}_{1}, \hat{c}_{2}$ e $\hat{c}_{3}$, e que o lugar das raízes é simétrico em relação ao eixo $j w$, deve-se escolher um dos pares de "zeros auxiliares" $\left(\hat{z}_{1} e \hat{z}_{2}\right.$ ou $\left.\hat{z}_{3} e \hat{z}_{4}\right)$ no semiplano esquerdo. Neste projeto optou-se pelo par $\hat{z}_{1} e \hat{z}_{2}$. Deste modo, tem-se que:

$$
\left\{\begin{array} { l } 
{ \hat { z } _ { 1 } + \hat { z } _ { 2 } = \frac { \hat { c } _ { 1 } } { \hat { c } _ { 2 } } } \\
{ \hat { z } _ { 1 } \hat { z } _ { 2 } = - \frac { \hat { c } _ { 3 } } { \hat { c } _ { 2 } } }
\end{array} \Rightarrow \left\{\begin{array}{l}
\hat{c}_{1}=\left(\hat{z}_{1}+\hat{z}_{2}\right) \hat{c}_{2} \\
\hat{c}_{3}=-\left(\hat{z}_{1} \hat{z}_{2}\right) \hat{c}_{2}
\end{array}\right.\right.
$$

A escolha de $\hat{z}_{1}$ e $\hat{z}_{2}$ é feita iterativamente para atender as condições de projeto, definidas no item 5.1. Deste modo tem-se:

$$
\hat{z}_{1}=\hat{z}_{2}=-32 \text {. }
$$

Da equação (5.24), assumindo $\hat{c}_{2}=1$, tem-se: $\hat{c}_{1}=-64$ e $\hat{c}_{3}=-1024$.

Os zeros $\hat{z}_{1}$ e $\hat{z}_{2}$ são simétricos a $\hat{z}_{3}$ e $\hat{z}_{4}$, então: $\hat{z}_{3}=-\hat{z}_{1}$ e $\hat{z}_{4}=-\hat{z}_{2}$.

Definidos os “zeros auxiliares" $(-32 ;-32 ; 32 ; 32)$ e os pólos $(0 ; 0 ; 0 ; 0 ; 0 ; 0)$ de malha aberta, esboça-se, em função do parâmetro $1 / \rho$, o lugar das raízes. A figura 5.6 apresenta este lugar das raízes e a localização dos pólos de malha fechada. 


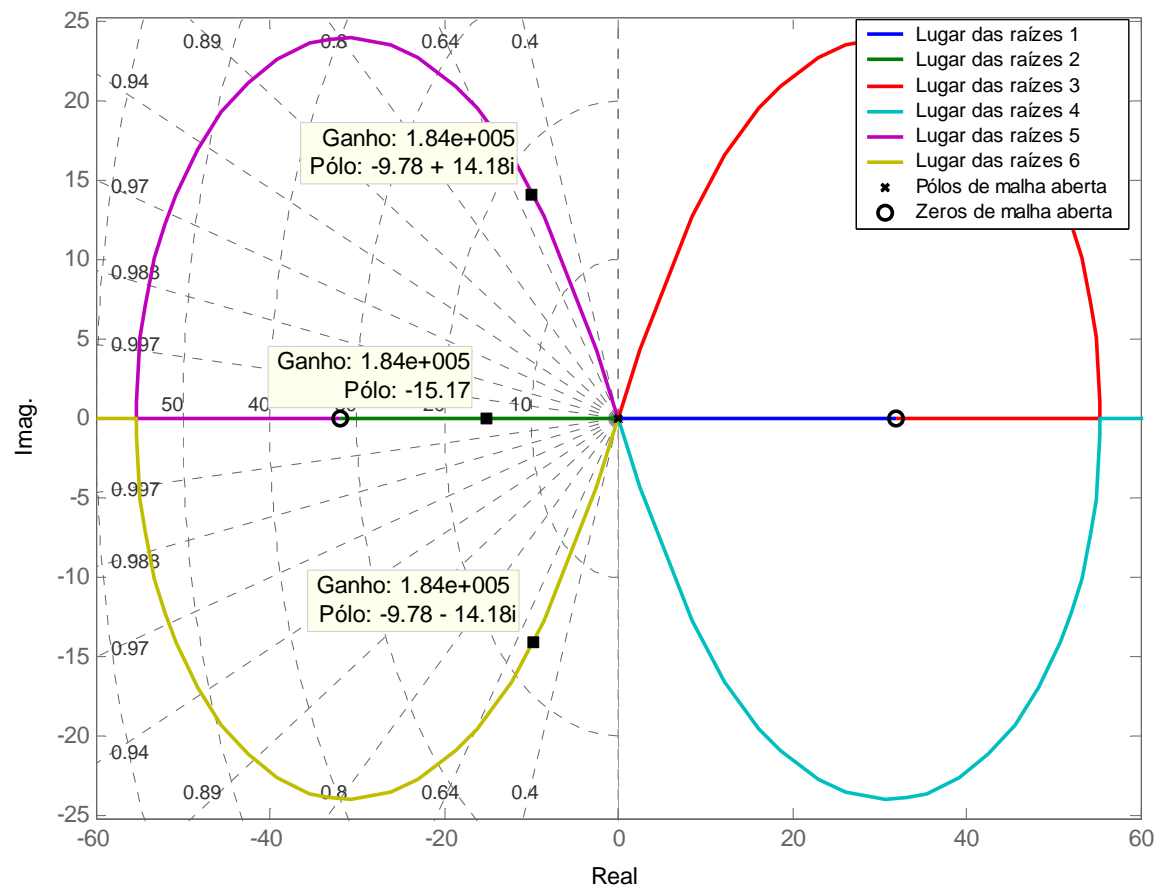

Figura 5.6: Lugar das raízes para projeto do controle vertical.

Considerando-se apenas o semiplano esquerdo, definem-se os pólos de malha fechada do RLQ que atendem as especificações de projeto, apresentadas no item 5.1:

$$
p_{1}=-9,78+14,18 j ; \quad p_{2}=-9,78-14,18 j ; \quad p_{3}=-15,17
$$

e obtém-se o valor do parâmetro $\rho$, ou seja:

$$
\rho=\frac{1}{184000} \cong 5,43 \cdot 10^{-6}
$$

Através da equação (4.8) calcula-se a matriz $Q$, como:

$$
Q=\left[\begin{array}{ccc}
4096 & -64 & 65536 \\
-64 & 1 & -1024 \\
65536 & -1024 & 1048576
\end{array}\right]
$$


Utilizando a função lqr do MATLAB, onde $K=\operatorname{lqr}(A, B, Q, \rho)$, calculam-se os ganhos de realimentação de estado, ou seja:

$$
K_{1 z}=57919,19 ; \quad K_{2 z}=-3389,66 ; \quad K_{3 z}=439247,06 .
$$

Conforme informado anteriormente, o sistema em malha fechada, equação (5.13), apresenta um zero de valor $\left(-K_{3 z} / K_{1 z}=-7,58\right)$, que provoca um sobre-sinal maior na resposta transitória.

Diante deste fato, se estivéssemos considerando apenas o critério de desempenho relativo à entrada de referência e não à perturbação, a idéia seria escolher um dos pólos de malha fechada para cancelar este zero de malha fechada. Escolhendo-se um dos "zeros auxiliares" de malha aberta, por exemplo $\hat{z}_{2}=-0,4$, quando se aumenta o ganho $(1 / \rho)$, o pólo e zero de malha fechada $\left(-K_{3 z} / K_{1 z}\right)$ tendem para o valor de $\hat{z}_{2}=-0,4$. Com isto há o cancelamento do pólo e zero, e o sistema é regido apenas pelos pólos complexos. Este controlador apresentaria um bom desempenho na partida do sistema, com um pequeno sobre-sinal.

Mas como o objetivo do controlador é rejeitar a perturbação, quando considerase a equação (5.16), verifica-se que um valor muito pequeno como pólo de malha fechada leva a um grande tempo de acomodação, pois este pólo não é cancelado.

\subsubsection{Controlador do movimento de rolagem}

Primeiro escolhe-se a matriz de projeto $(\hat{C})$, como sendo:

$$
\hat{C}=\left[\begin{array}{lll}
\hat{c}_{1} & \hat{c}_{2} & \hat{c}_{3}
\end{array}\right]
$$

Calcula-se a função de transferência $G_{O L}(s)$ e $G_{O L}(-s)$, ou seja:

$$
G_{O L}(s)=\frac{b(s)}{a(s)}=\hat{C}(s I-A)^{-1} B=\frac{\hat{c}_{2}\left(s^{2}-\frac{\hat{c}_{1}}{\hat{c}_{2}} s-\frac{\hat{c}_{3}}{\hat{c}_{2}}\right)}{I_{x x} s^{3}}
$$




$$
G_{O L}(-s)=\frac{b(-s)}{a(-s)}=\hat{C}(-s I-A)^{-1} B=\frac{-\hat{c}_{2}\left(s^{2}+\frac{\hat{c}_{1}}{\hat{c}_{2}} s-\frac{\hat{c}_{3}}{\hat{c}_{2}}\right)}{I_{x x} s^{3}} .
$$

Reescrevendo na forma de pólos e zeros, tem-se que:

$$
\frac{b(-s) b(s)}{a(-s) a(s)}=-\left(\frac{\hat{c}_{2}}{I_{x x}}\right)^{2} \frac{\left(s-\hat{z}_{1}\right)\left(s-\hat{z}_{2}\right)\left(s-\hat{z}_{3}\right)\left(s-\hat{z}_{4}\right)}{s^{6}}
$$

onde:

$$
\hat{z}_{1,2}=\frac{-\hat{c}_{1} \pm \sqrt{\hat{c}_{1}^{2}+4 \hat{c}_{2} \hat{c}_{3}}}{2 \hat{c}_{2}} \text { e } \hat{z}_{3,4}=\frac{\hat{c}_{1} \pm \sqrt{\hat{c}_{1}^{2}+4 \hat{c}_{2} \hat{c}_{3}}}{2 \hat{c}_{2}}
$$

Visto que estes "zeros auxiliares" apresentam a mesma estrutura do controle do movimento vertical, a escolha de $\hat{z}_{1}$ e $\hat{z}_{2}$ é feita recursivamente para atender as condições de projeto, definidas no item 5.1. Deste modo tem-se:

$$
z_{1}=z_{2}=-32
$$

Da equação (5.24), assumindo $\hat{c}_{2}=1$, tem-se: $\hat{c}_{1}=-64$ e $\hat{c}_{3}=-1024$.

Os zeros $\hat{z}_{1}$ e $\hat{z}_{2}$ são simétricos a $\hat{z}_{3}$ e $\hat{z}_{4}$, então: $\hat{z}_{3}=-\hat{z}_{1}$ e $\hat{z}_{4}=-\hat{z}_{2}$.

Definidos os "zeros auxiliares" $(-32 ;-32 ; 32 ; 32)$ e os pólos $(0 ; 0 ; 0 ; 0 ; 0 ; 0)$ de malha aberta, esboça-se, em função do parâmetro $1 / \rho$, o lugar das raízes. A estrutura do lugar das raízes é igual à do movimento vertical, figura 5.6.

Considerando-se apenas o semiplano esquerdo, definem-se os pólos de malha fechada do RLQ que atendem as especificações de projeto, apresentadas no item 5.1:

$$
p_{1}=-9,86+14,26 j ; \quad p_{2}=-9,86-14,26 j ; \quad p_{3}=-15,24
$$

e obtém-se o valor do parâmetro $\rho$, ou seja: 


$$
\rho=\frac{1}{700} \cong 0,00143
$$

Através da equação (4.8) calcula-se a matriz $Q$, como:

$$
Q=\left[\begin{array}{ccc}
4096 & -64 & 65536 \\
-64 & 1 & -1024 \\
65536 & -1024 & 1048576
\end{array}\right]
$$

Utilizando a função lqr do MATLAB, calculam-se os ganhos de realimentação de estado, ou seja:

$$
K_{1 \phi}=-3555,04 ; \quad K_{2 \phi}=206,79 ; \quad K_{3 \phi}=-27092,49 .
$$

\subsubsection{Controlador do movimento de arfagem}

O projeto do controlador do movimento de arfagem é similar ao do movimento de rolagem, alterando-se o movimento de inércia para $I_{y y}$.

Foram escolhidos os mesmos valores dos "zeros auxiliares" e pólos de malha aberta. Deste modo o formato do lugar das raízes é igual ao apresentado na figura 5.6. Considerando-se apenas o semiplano esquerdo, definem-se os pólos de malha fechada do RLQ que atendem as especificações de projeto, apresentadas no item 5.1:

$$
p_{1}=-11,42+15,81 j ; \quad p_{2}=-11,42-15,81 j ; \quad p_{3}=-16,66
$$

e obtém-se o valor do parâmetro $\rho$, ou seja:

$$
\rho=\frac{1}{1340} \cong 1,46.10^{-4}
$$

A matriz $Q$ tem o mesmo valor do movimento de rolagem, ou seja: 


$$
Q=\left[\begin{array}{ccc}
4096 & -64 & 65536 \\
-64 & 1 & -1024 \\
65536 & -1024 & 1048576
\end{array}\right]
$$

Os ganhos de realimentação de estado calculados são:

$$
K_{1 \theta}=-4875,37 ; \quad K_{2 \theta}=280,45 ; \quad K_{3 \theta}=-37484,55
$$

\subsection{Controlador RLQ para o modelo de corpo não rígido}

Considerando o modelo de corpo não rígido, foi projetado um controlador para cada movimento. A figura 5.7 mostra o diagrama de blocos do sistema em malha fechada, com um controlador independente para cada grau de liberdade.

Os controladores dos movimentos: vertical, de rolagem e de arfagem são os mesmos controladores do modelo de corpo rígido (item 5.4). Foi incluído apenas o controle do movimento de torção, conforme definido a seguir.

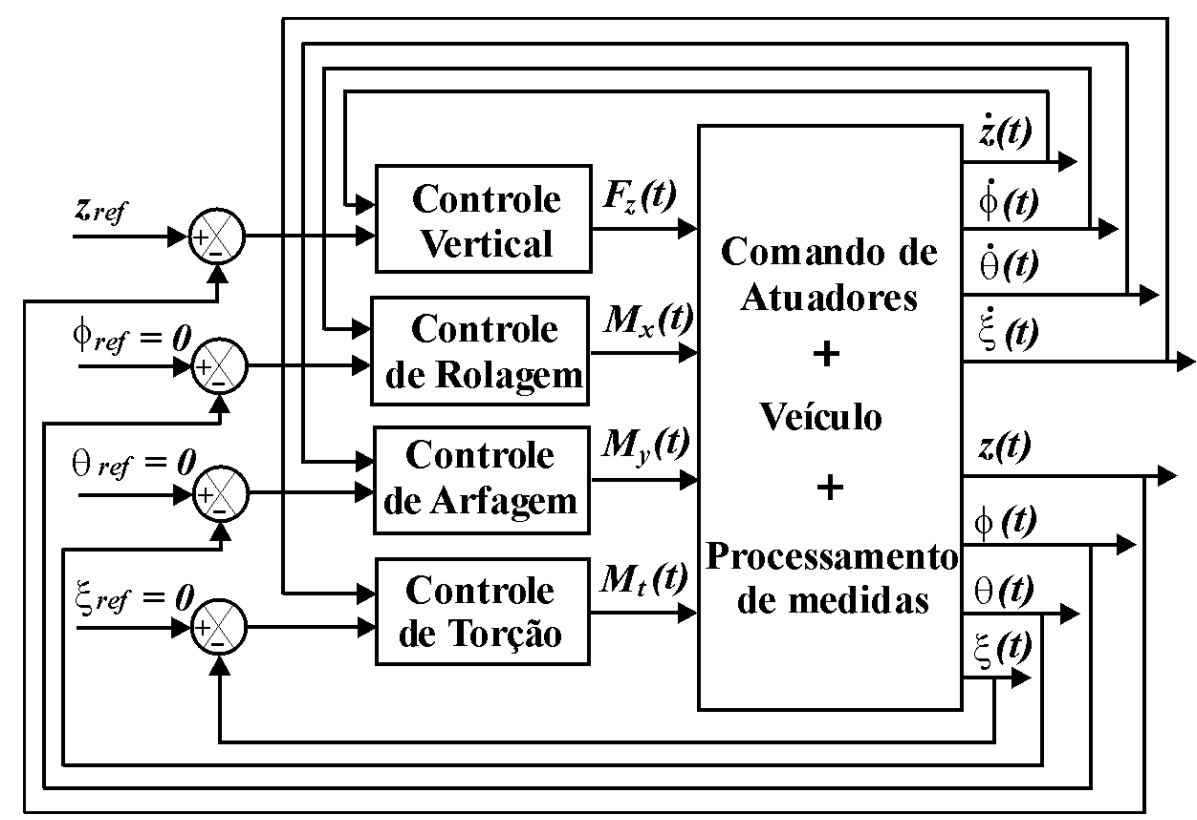

Figura 5.7: Sistema em malha fechada - modelo de corpo não rígido. 


\subsubsection{Controlador do movimento de torção}

O movimento de torção é similar ao movimento de rolagem e o momento de inércia $I_{t} \cong I_{x x}$, conforme apresentado no item 3.1.2. Mas, na verdade, existe uma força de resistência que se opõe ao movimento de torção. Deste modo, no cálculo dos ganhos de realimentação de estados do controlador, o tempo de acomodação deve ser menor do que do movimento de rolagem.

Os valores de $\hat{z}_{1} e \hat{z}_{2}$ que atendem as restrições de projeto são:

$$
\hat{z}_{1}=\hat{z}_{2}=-40
$$

Da equação (5.24), assumindo $\hat{c}_{2}=1$, tem-se: $\hat{c}_{1}=-80$ e $\hat{c}_{3}=-1600$.

Os "zeros auxiliares" $\hat{z}_{3}$ e $\hat{z}_{4}$ são: $\hat{z}_{3}=-\hat{z}_{1}$ e $\hat{z}_{4}=-\hat{z}_{2}$.

Definidos os “zeros auxiliares" $(-40 ;-40 ; 40 ; 40)$ e os pólos $(0 ; 0 ; 0 ; 0 ; 0 ; 0)$ de malha aberta, esboça-se, em função do parâmetro $1 / \rho$, o lugar das raízes. A estrutura do lugar das raízes é igual à do movimento vertical, figura 5.6.

Os pólos de malha fechada do RLQ que atendem as restrições de projeto são:

$$
p_{1}=-13,79+19,30 j ; \quad p_{2}=-13,79-19,30 j ; \quad p_{3}=-20,40
$$

e o valor do parâmetro $\rho$ é:

$$
\rho=\frac{1}{1800} \cong 5,56 \cdot 10^{-4}
$$

Calculando-se a matriz $Q$ tem-se que:

$$
Q=\left[\begin{array}{ccc}
6400 & -80 & 128000 \\
-80 & 1 & -1600 \\
128000 & -1600 & 2560000
\end{array}\right]
$$

Os ganhos de realimentação calculados são:

$$
K_{1 \xi}=-6654,96 ; \quad K_{2 \xi}=283,79 ; \quad K_{3 \xi}=-67882,25 \text {. }
$$




\subsection{Controlador RLQ para o Modelo SISO}

O modelo SISO descrito pela equação (3.8) tem o sistema de equações formado por quatro movimentos verticais, idênticos, e descritos no item 5.2.1.

Deve-se considerar que o valor da massa agora e um quarto da massa total do veículo, ou seja $\frac{M}{4}$.

A figura 5.8 mostra o diagrama de blocos do sistema em malha fechada, com um controlador independente para cada controle vertical.

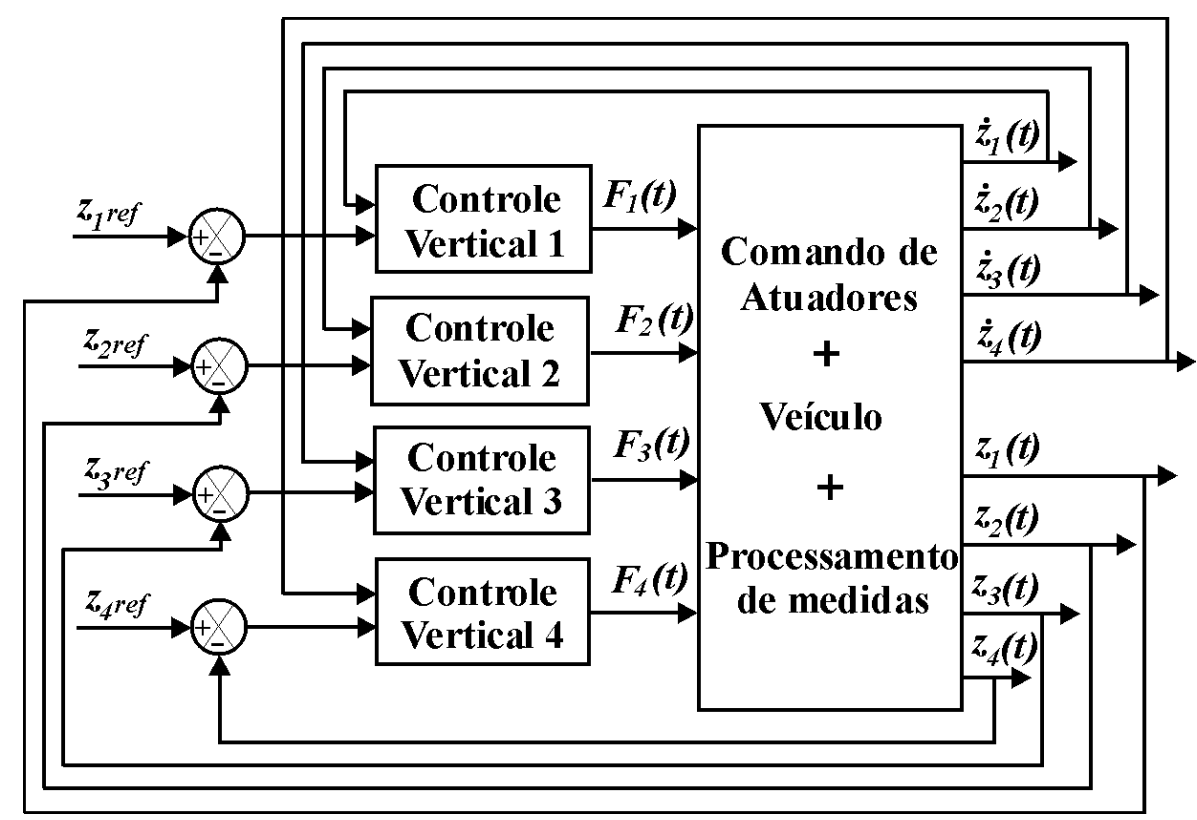

Figura 5.8: Sistema em malha fechada - modelo SISO.

O projeto do controlador do movimento vertical é similar ao apresentado no item 5.4.1, lembrado que a nova massa é $\frac{M}{4}$. Deste modo, tem-se como resultado da escolha iterativa de $\hat{z}_{1}$ e $\hat{z}_{2}$ os seguintes valores:

$$
\hat{z}_{1}=\hat{z}_{2}=-32 \text {. }
$$

Da equação (5.24), assumindo $\hat{c}_{2}=1$, tem-se: $\hat{c}_{1}=-64$ e $\hat{c}_{3}=-1024$. 
Os zeros $\hat{z}_{3}$ e $\hat{z}_{4}$ são: $\hat{z}_{3}=-\hat{z}_{1}$ e $\hat{z}_{4}=-\hat{z}_{2}$.

Definidos os "zeros auxiliares" $(-32 ;-32 ; 32 ; 32)$ e os pólos $(0 ; 0 ; 0 ; 0 ; 0 ; 0)$ de malha aberta, o esboço do lugar das raízes será igual ao apresentado na figura 5.6.

Considerando-se apenas o semiplano esquerdo, definem-se os pólos de malha fechada do RLQ que atendem as especificações de projeto, apresentadas no item 5.1:

$$
p_{1}=-10,10+14,51 j ; \quad p_{2}=-10,10-14,51 j ; \quad p_{3}=-15,48
$$

e obtém-se o valor do parâmetro $\rho$, ou seja:

$$
\rho=\frac{1}{13300} \cong 7,52 \cdot 10^{-5}
$$

A matriz $Q$ tem o mesmo valor da do movimento vertical, ou seja:

$$
Q=\left[\begin{array}{ccc}
4096 & -64 & 65536 \\
-64 & 1 & -1024 \\
65536 & -1024 & 1048576
\end{array}\right]
$$

Os ganhos de realimentação de estado são:

$$
K_{1 z}=15260,04 ; \quad K_{2 z}=-870,63 ; \quad K_{3 z}=118093,44
$$

\subsection{Controlador $\mathbf{H}_{2} / \mathbf{H}_{\infty}$ para o Modelo com realimentação da saída}

Considerando o modelo rígido com realimentação da saída, o sistema de equações é formado pelos movimentos vertical, de rolagem e de arfagem. Sendo assim, foi projetado um controlador para cada movimento.

Este modelo é definido pelas equações (3.1), (3.2) e (3.3), sendo similar ao modelo rígido do item 3.1, mas com o controle da levitação realizado pela realimentação da saída ao invés da realimentação de estados. 
Na figura 5.9 tem-se o diagrama de blocos do sistema em malha fechada, conforme apresentado em (BITTAR, 1998; COSTA et al., 2003), com um controlador independente para cada grau de liberdade.

O modelo dinâmico do sistema tem três entradas $\left(F_{\mathrm{z}}(t), M_{\mathrm{x}}(t)\right.$ e $\left.M_{\mathrm{y}}(t)\right)$ e três saídas $(z(t), \phi(t) e \theta(t))$.

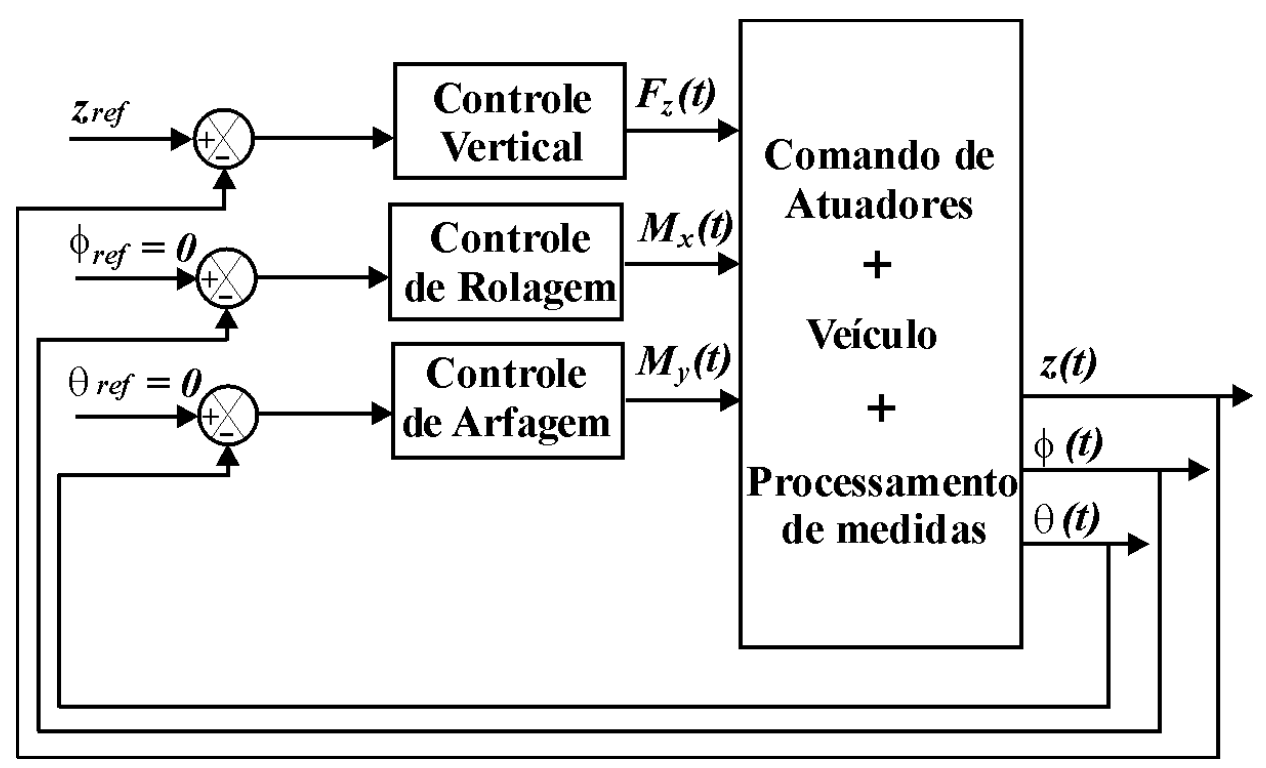

Figura 5.9: Sistema em malha fechada - modelo de corpo rígido com realimentação da saída.

Os controladores foram projetados utilizando a teoria $\mathrm{H}_{2} / \mathrm{H}_{\infty}$ (DOYLE, 1989). Suas funções de transferência (BITTAR, 1998; COSTA et al., 2003) são dadas por:

$$
G_{c}(s)=\frac{K_{c}(s+0,8)(s+60,0)(s+62,6)(s+100,0)}{s(s+57,5)(s+71,5)(s+301,7 \pm j 299,2)}
$$

onde o ganho $K_{\mathrm{c}}$ é sintonizado experimentalmente para cada controlador de modo a estabilizar o sistema em malha fechada.

As importantes características de $G_{\mathrm{c}}(s)$ são:

- avanço-atraso de fase; 
- ocorrência de avanço de fase em baixas freqüências, que é essencial para produzir estabilidade de malha fechada, dado que o modelo de malha aberta possui pólo na origem;

- presença de pólos de alta freqüência para rejeitar ruído de sensor;

- presença de integrador para obter erro nulo no estado estacionário para entradas a degrau na referência (partida do sistema de levitação) e para perturbações a degrau na entrada da planta (variações de massa).

\subsection{Simulação}

Neste item estão apresentados os resultados das simulações dos controladores RLQ desenvolvidos.

As simulações e a implementação dos modelos e dos controladores foram desenvolvidas utilizando-se o software SIMULINK.

As figuras 5.10, 5.11 e 5.12, respectivamente, apresentam os diagramas de blocos dos sistemas utilizados para a simulação dos controles vertical, de rolagem e arfagem. Devido às similaridades, a simulação do controle de torção foi realizada utilizando-se o diagrama da figura 5.11. A simulação do movimento vertical SISO foi realizada com a estrutura do diagrama da figura 5.10 com os ganhos (equação (5.47)) e massa $\left(\frac{M}{4}\right)$ pertinentes.

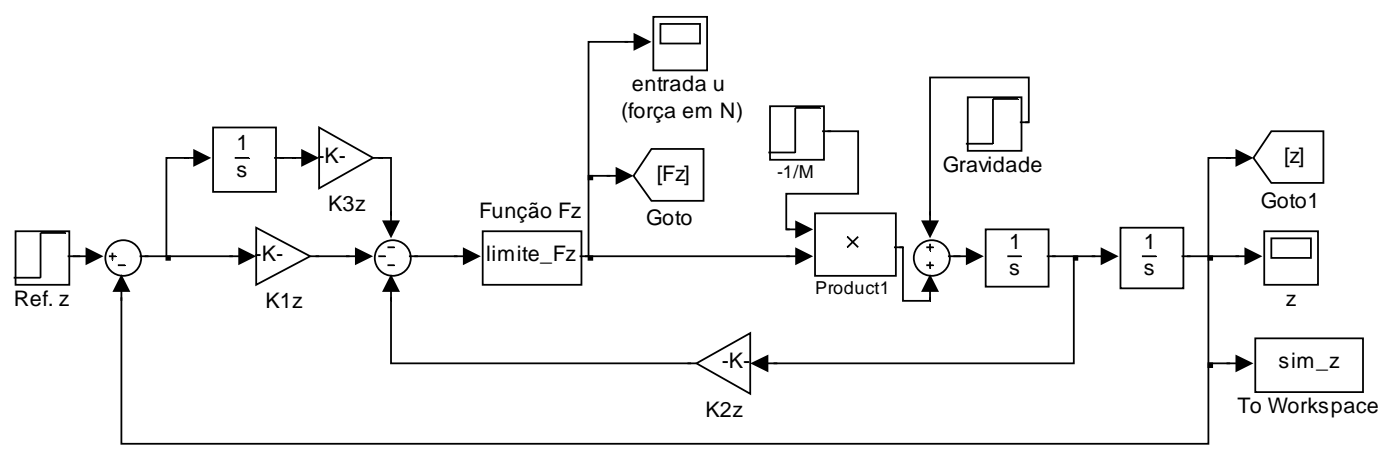

Figura 5.10: Diagrama de blocos para simulação do controle vertical. 
$\mathrm{Na}$ tentativa de tornar a simulação mais próxima da realidade, foram implementadas as funções "limite_Fz", "limite_Mx" e "limite_My" para limitação, conforme equações (3.23) e (3.39).

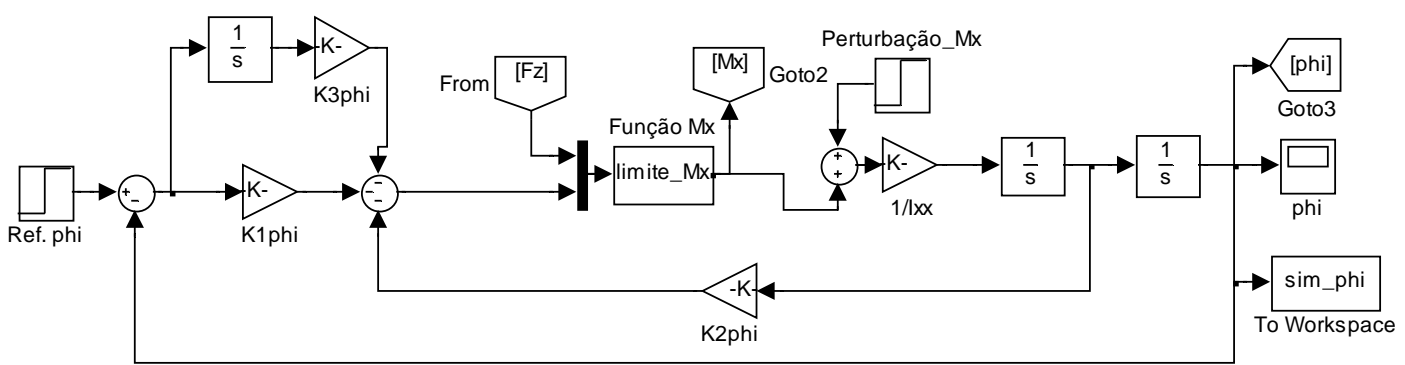

Figura 5.10: Diagrama de blocos para simulação do controle de rolagem.

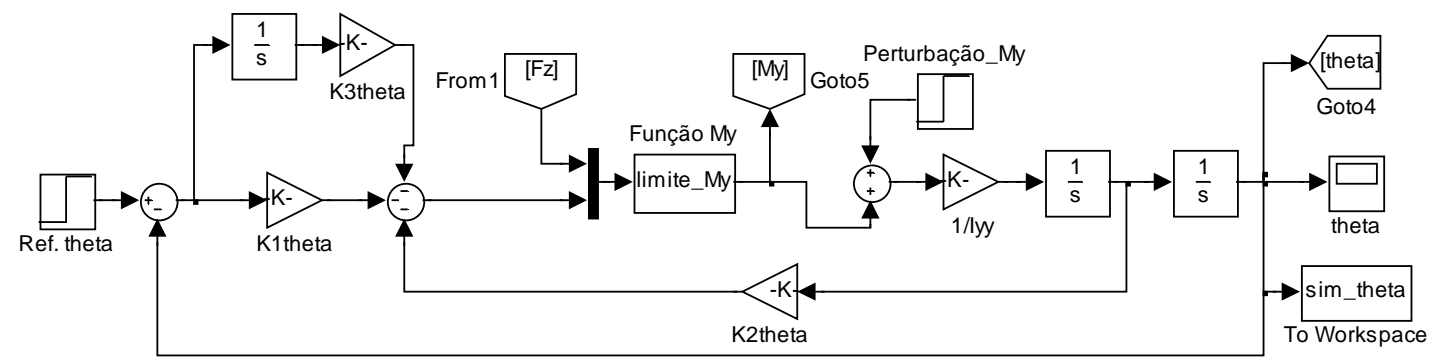

Figura 5.12: Diagrama de blocos para simulação do controle de arfagem.

Através do diagrama de blocos da figura 5.13 é possível simular as tensões aplicadas nos atuadores.

Na função "otm_forca" está inserido o procedimento de otimização das forças de suspensão para o modelo rígido, conforme item 3.3. No caso do cálculo da distribuição das forças para o modelo não rígido são acrescidas, ao diagrama de blocos e função "otm_forca", as informações do momento $M_{\mathrm{t}}$ e do ângulo de torção $(\xi)$. 
A função "tensão" calcula, através da equação (3.10), as tensões $v_{i}(t)(i=1, \ldots, 4)$ que são aplicadas nos atuadores ou circuito de potência.

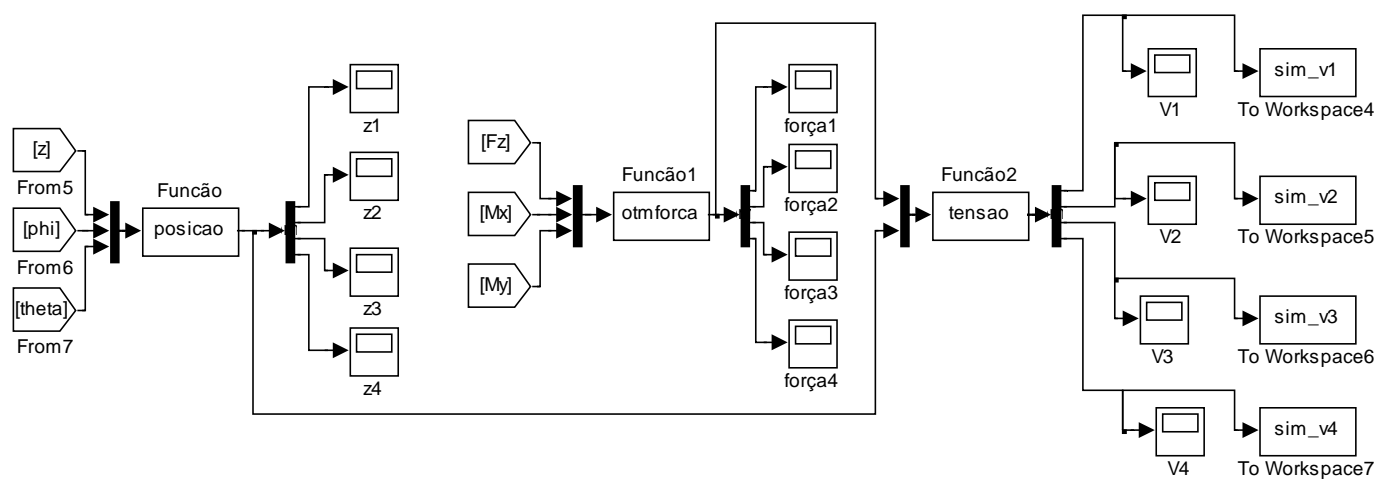

Figura 5.13: Diagrama de blocos para simulação das tensões $v_{i}(t)$ do atuador.

Estando o sistema de corpo rígido em regime estacionário $(z=7,5 \mathrm{~mm} \mathrm{e}$ $\phi=\theta=0 \mathrm{rad})$, no instante de tempo igual a $2 \mathrm{~s}$ foi aplicado um degrau de $2,5 \mathrm{~mm}$ na entrada do movimento vertical e no instante $3 s$ foi aplicada uma perturbação de massa $(10 \mathrm{~kg})$.

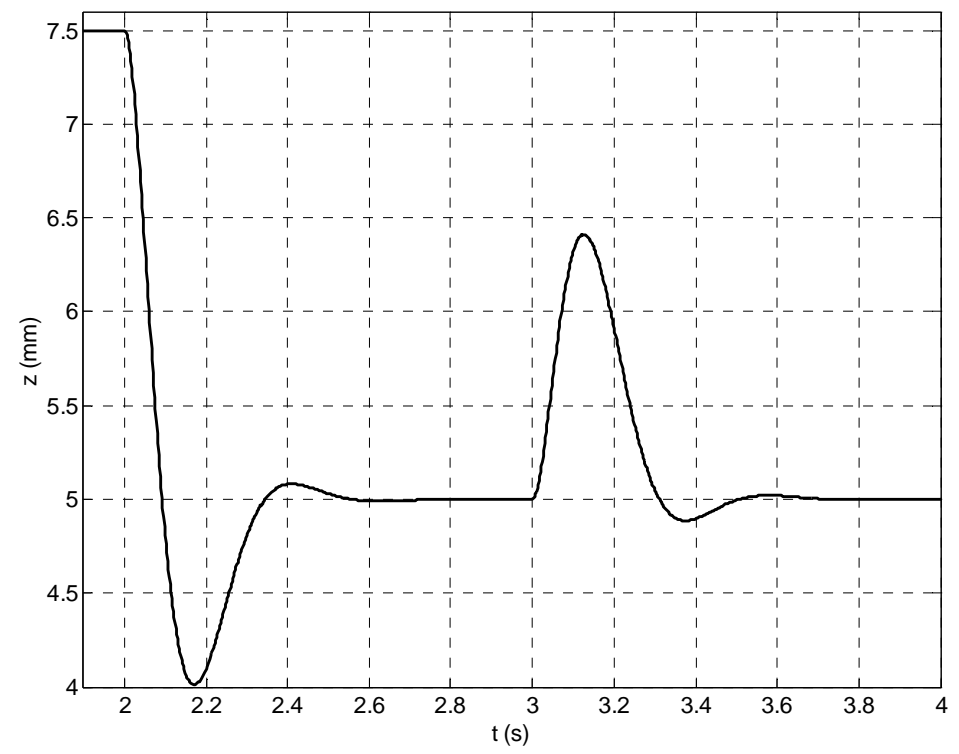

Figura 5.14: Resposta ao degrau e perturbação para o controle vertical. 
Analisando a resposta transitória da figura 5.14, tem-se que:

$$
t_{r}=0,092 s ; \quad t_{s}=0,48 s ; \quad M_{p}=39,6 \% ; \quad d=1,4 m m \quad t_{p}<0,7 s ;(5.49)
$$

onde:

- $t_{\mathrm{r}}$ é o tempo de subida, ou seja, o tempo necessário para a resposta passar de $0 \%$ a $100 \%$ do seu valor final;

- $t_{\mathrm{s}}$ é o tempo de acomodação, ou seja, o tempo necessário para a curva de resposta alcançar e permanecer dentro de um faixa em torno de $2 \%$ do valor final;

- $M_{\mathrm{p}}$ é o sobre-sinal máximo em valor percentual;

- $d_{e}$ é o valor do máximo desvio em relação ao valor em regime estacionário devido à perturbação;

- $t_{\mathrm{p}}$ é o tempo de acomodação devido à perturbação.
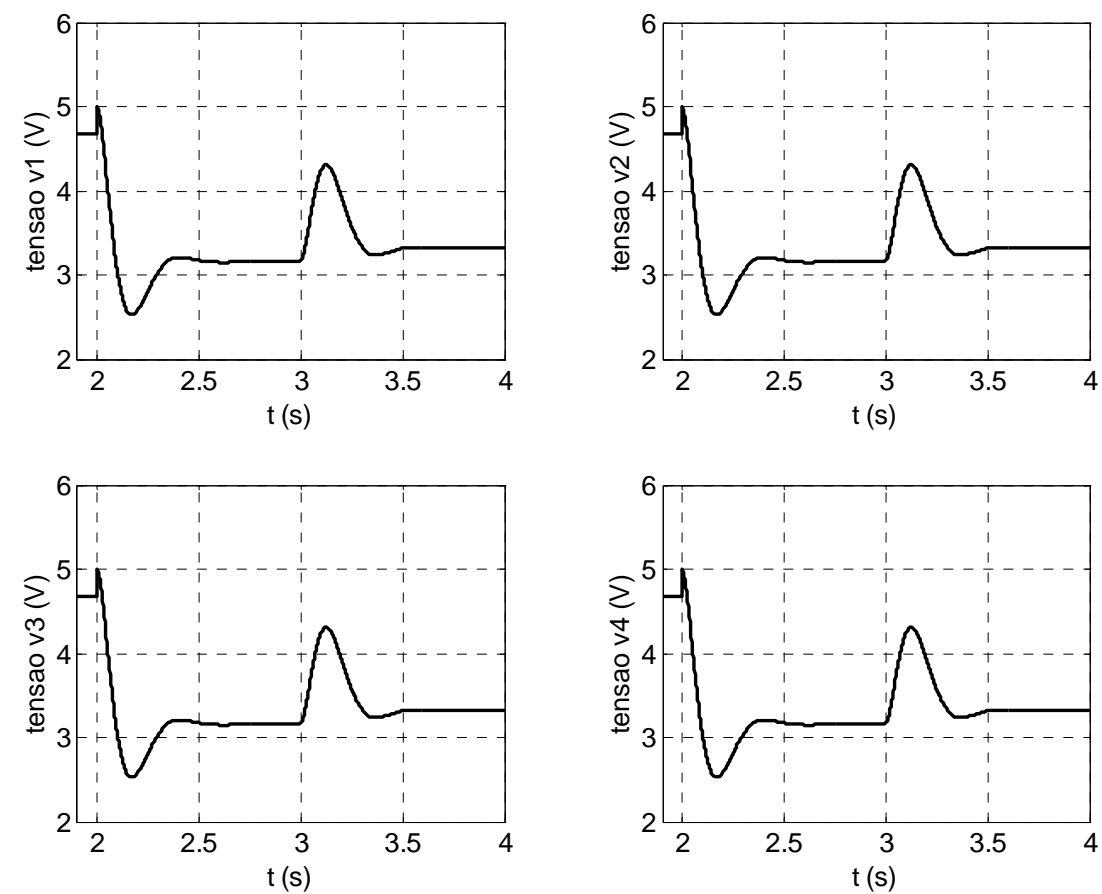

Figura 5.15: Tensões nos atuadores para o controle vertical. 
Verifica-se através da figura 5.15 que as tensões dos atuadores não são maiores que $5 \mathrm{~V}$, conforme especificações de projeto.

Com o sistema em regime estacionário $(z=5,0 \mathrm{~mm}, \phi=-0,008 \mathrm{rad}$ e $\theta=0 \mathrm{rad})$, no instante de tempo igual a $2 s$ foi aplicado na entrada do movimento de rolagem um degrau de $0,008 \mathrm{rad}$ e no instante $3 \mathrm{~s}$ uma perturbação de momento $(25 \mathrm{Nm})$.

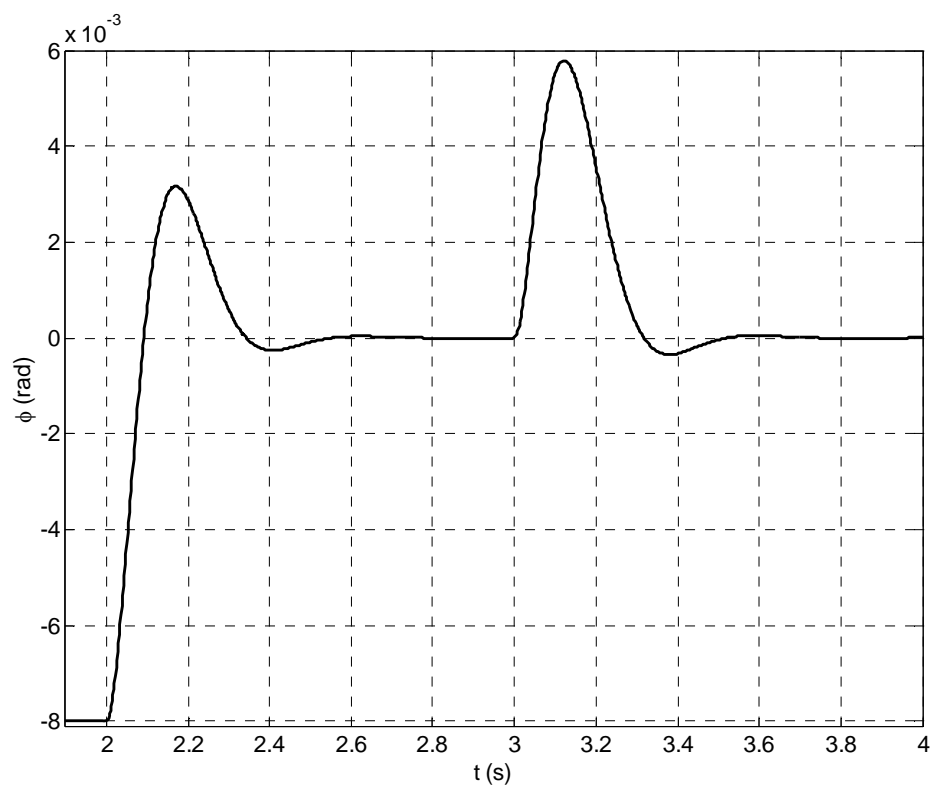

Figura 5.16: Resposta ao degrau e perturbação para o controle de rolagem.

Analisando a resposta transitória da figura 5.16 tem-se que:

$$
t_{r}=0,092 s ; \quad t_{s}=0,47 \mathrm{~s} ; \quad M_{p}=39,5 \% ; \quad d_{e}=0,0058 \mathrm{rad} ; \quad t_{p}<0,7 \mathrm{~s}(5.50)
$$

As tensões dos atuadores são menores que $5 \mathrm{~V}$ (figura 5.17).

Para movimento de arfagem, estando o sistema em regime estacionário ( $z=5,0 \mathrm{~mm}, \phi=0 \mathrm{rad}$ e $\theta=-0,008 \mathrm{rad}$ ), no instante de tempo igual a $2 s$ foi aplicado na entrada um degrau de 0,008rad e no instante $3 \mathrm{~s}$ uma perturbação de momento $(25 \mathrm{Nm})$. 

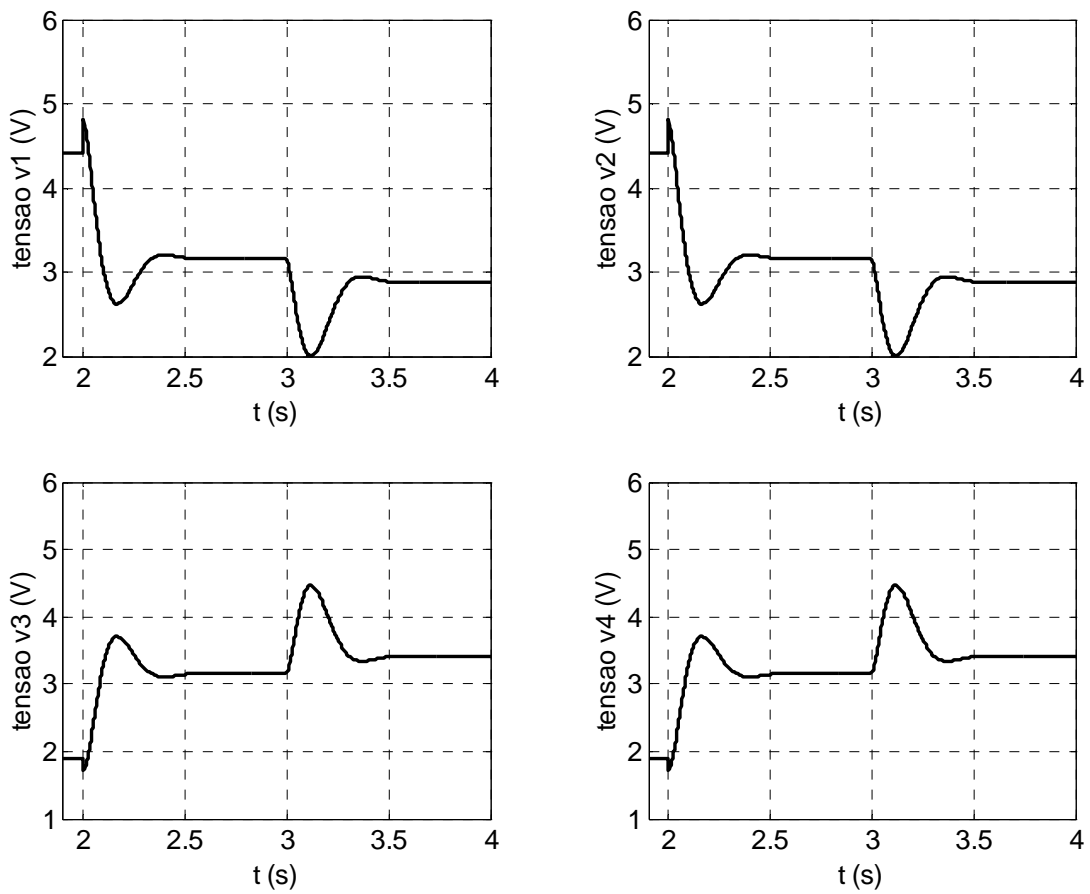

Figura 5.17: Tensões nos atuadores para o controle de rolagem.

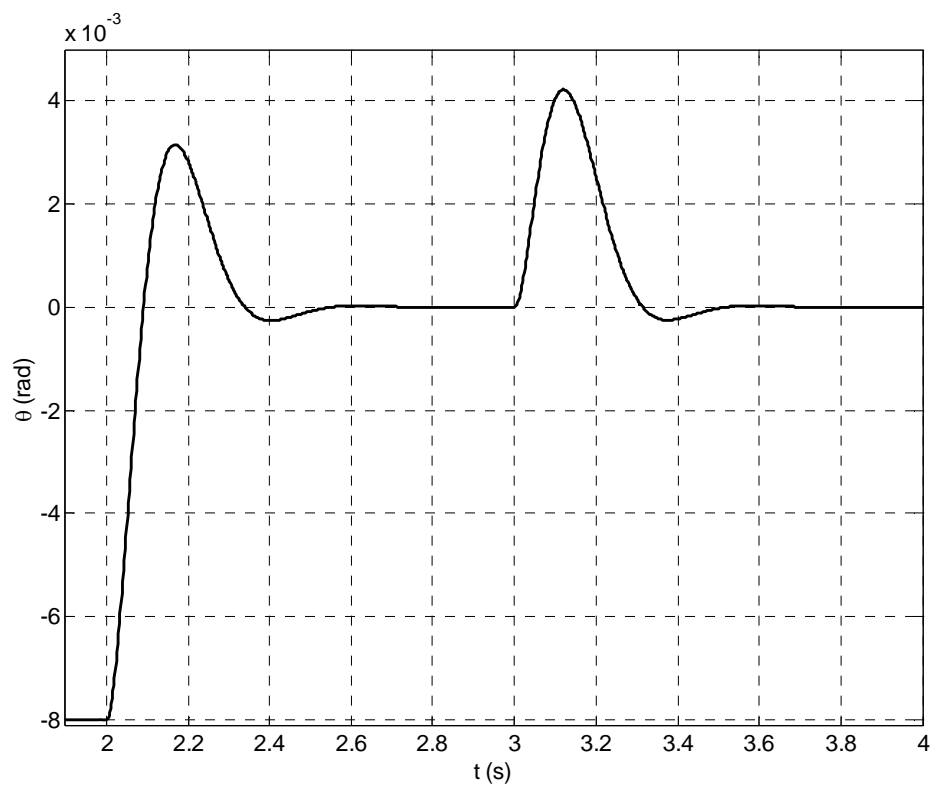

Figura 5.18: Resposta ao degrau e perturbação para o controle de arfagem. 
Analisando a resposta transitória da figura 5.18 tem-se que:

$$
t_{r}=0,091 s ; \quad t_{s}=0,46 s ; \quad M_{p}=39,5 \% ; \quad d_{e}=0,0042 ; \quad t_{p}<0,7 s ;(5.51)
$$

As tensões dos atuadores são menores que $5 V$ (figura 5.19).
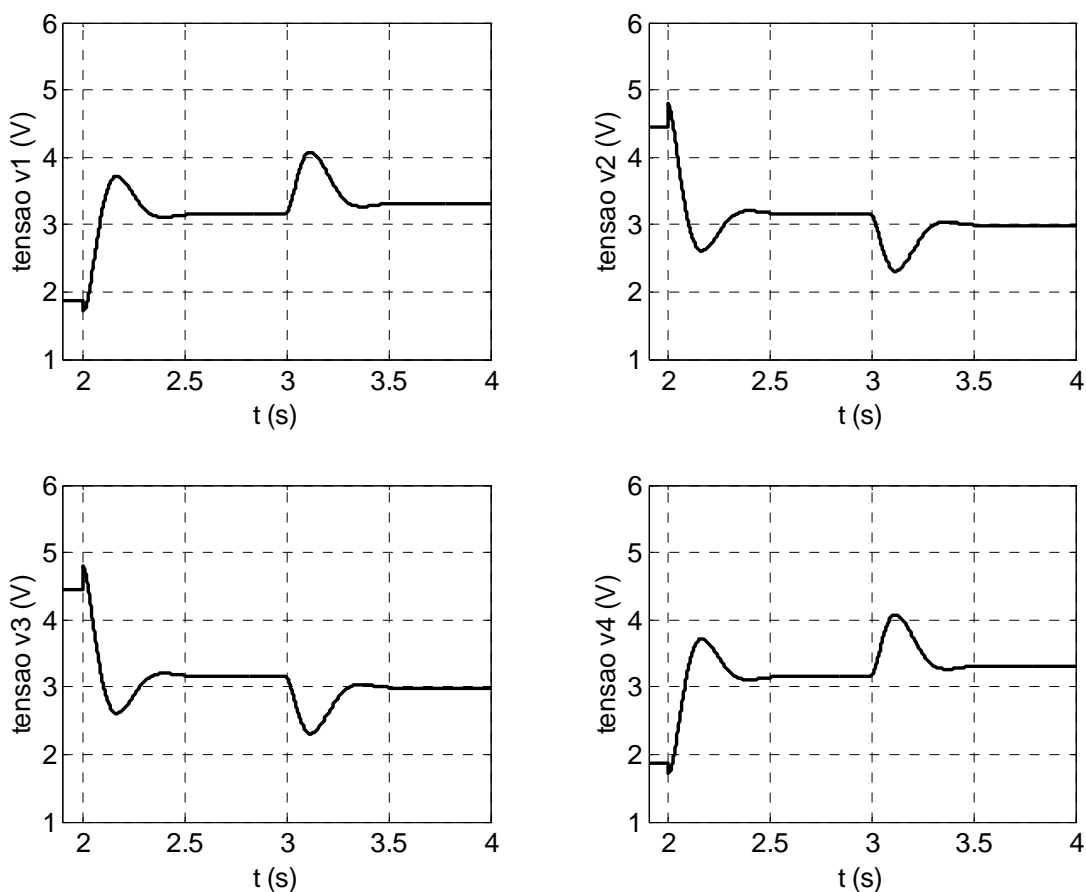

Figura 5.19: Tensões nos atuadores para o controle de arfagem.

Considerando o modelo de corpo não rígido e com o sistema em regime estacionário $(z=5,0 \mathrm{~mm}, \phi=\theta=0 \mathrm{rad}$ e $\xi=-0,008 \mathrm{rad})$ foi aplicado, no movimento de torção, no instante $2 s$ um degrau de $0,008 \mathrm{rad}$ na entrada, e no instante $3 s$ uma perturbação de momento $(25 \mathrm{Nm})$.

Analisando a resposta transitória da figura 5.20 tem-se que:

$$
t_{r}=0,068 s ; \quad t_{s}=0,34 s ; \quad M_{p}=38,9 \% ; \quad d_{e}=0,0031 ; \quad t_{p}<0,6 s ;(5.52)
$$




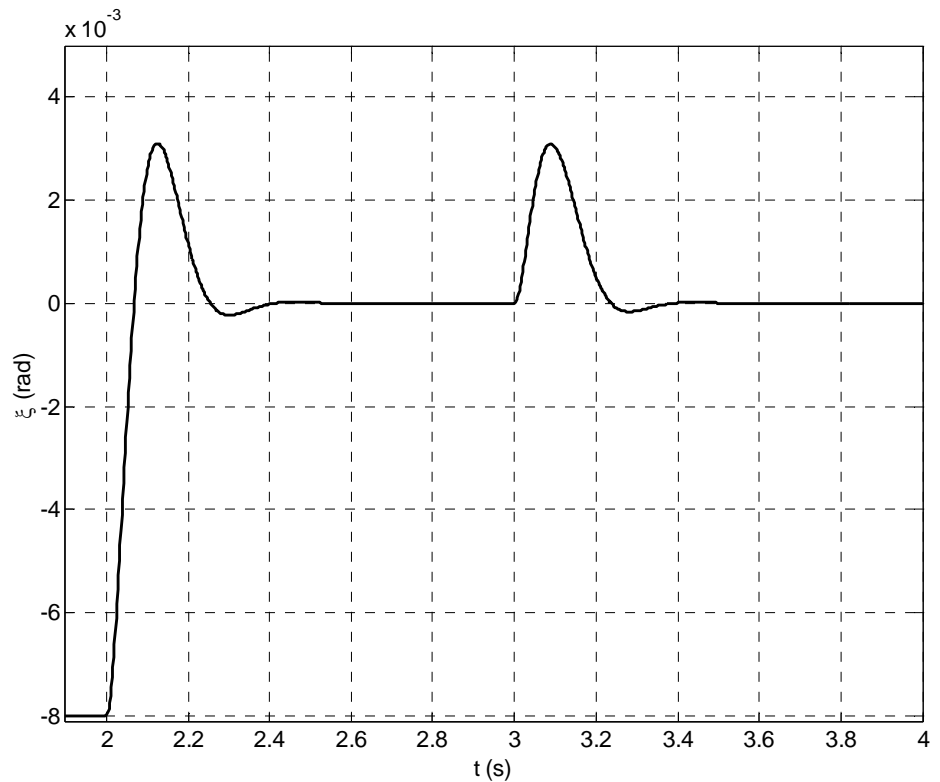

Figura 5.20: Resposta ao degrau e perturbação para o controle de torção.
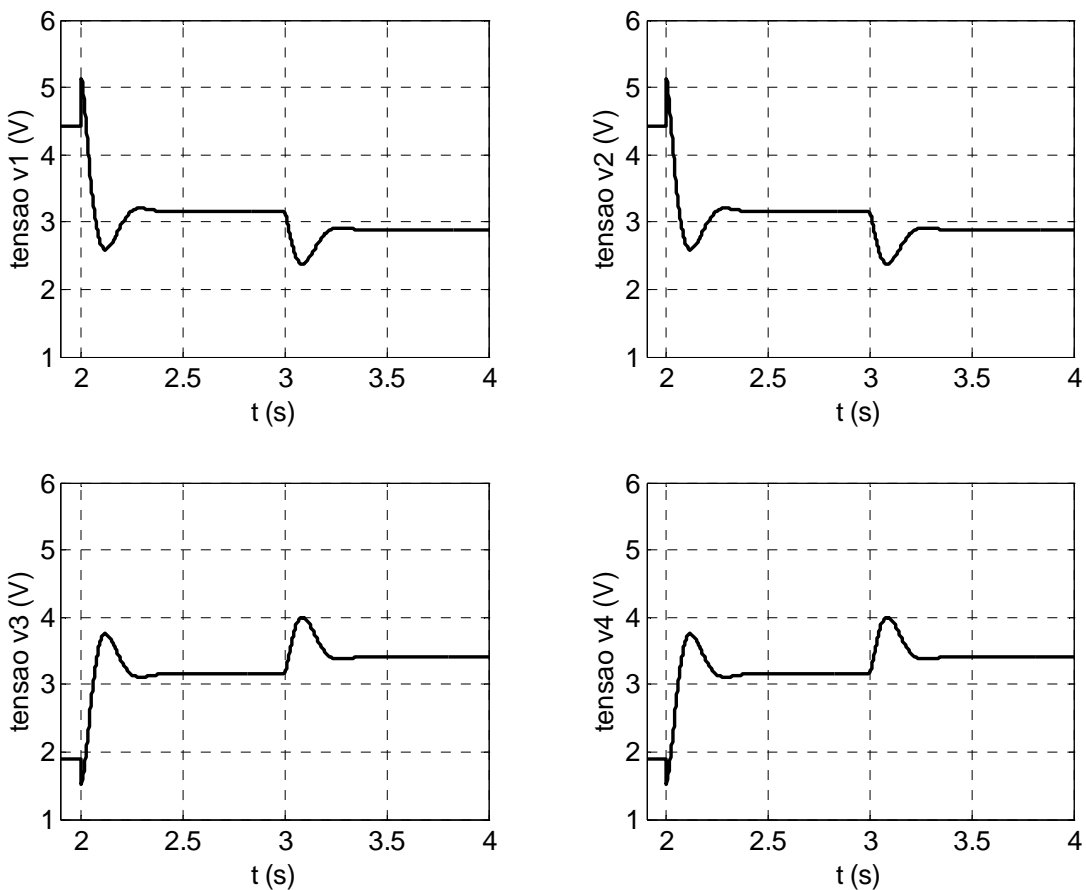

Figura 5.21: Tensões nos atuadores para o controle de torção. 
Apesar de a tensão do atuador ser maior que $5 V$ (figura 5.21), permaneceu-se com os ganhos de realimentação de estado, pois o degrau de 0,008rad está na prática sobre estimado.

Na simulação do modelo SISO, em um dos movimentos verticais, foi aplicado um degrau $(2,5 \mathrm{~mm})$ e perturbação de massa $(2,5 \mathrm{~kg})$, da mesma maneira que no movimento vertical do corpo rígido. Analisando a resposta transitória da figura 5.22, tem-se que:

$$
t_{r}=0,090 \mathrm{~s} ; \quad t_{s}=0,46 s ; \quad M_{p}=39,4 \% ; \quad d_{e}=1,4 m m ; \quad t_{p}<0,7 \mathrm{~s} ;(5.53)
$$

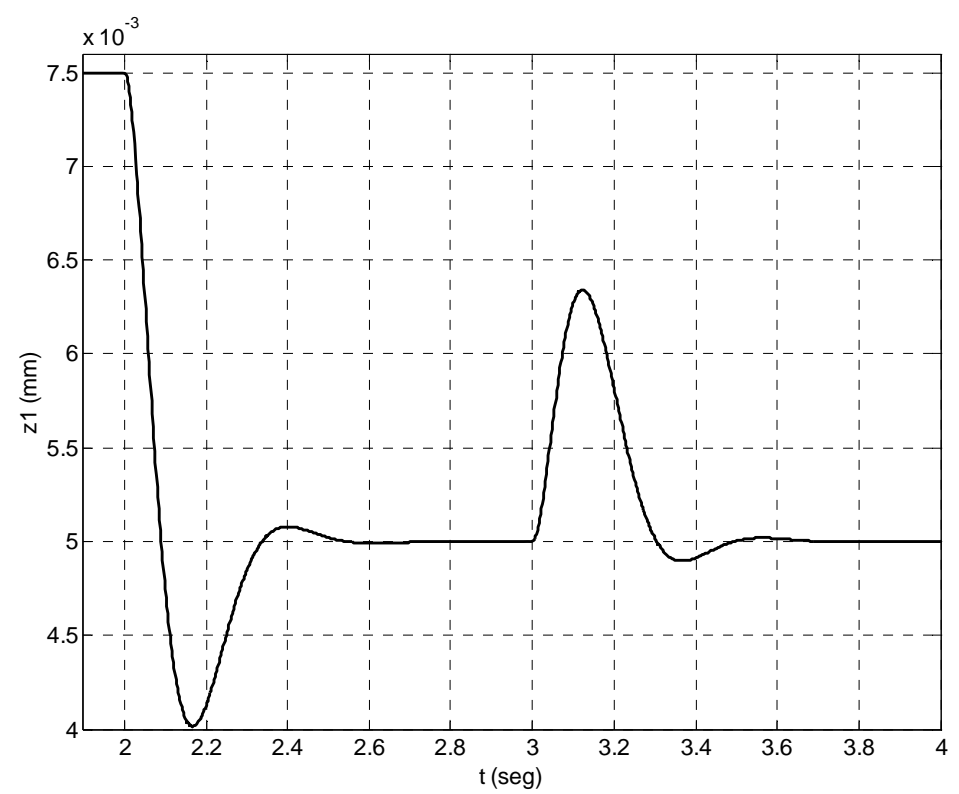

Figura 5.22: Resposta ao degrau e perturbação para o controle SISO. 
$\mathrm{Na}$ figura 5.23, verifica-se que a tensão $v_{1}(\mathrm{t})$, do atuador, é menor que $5 V$.

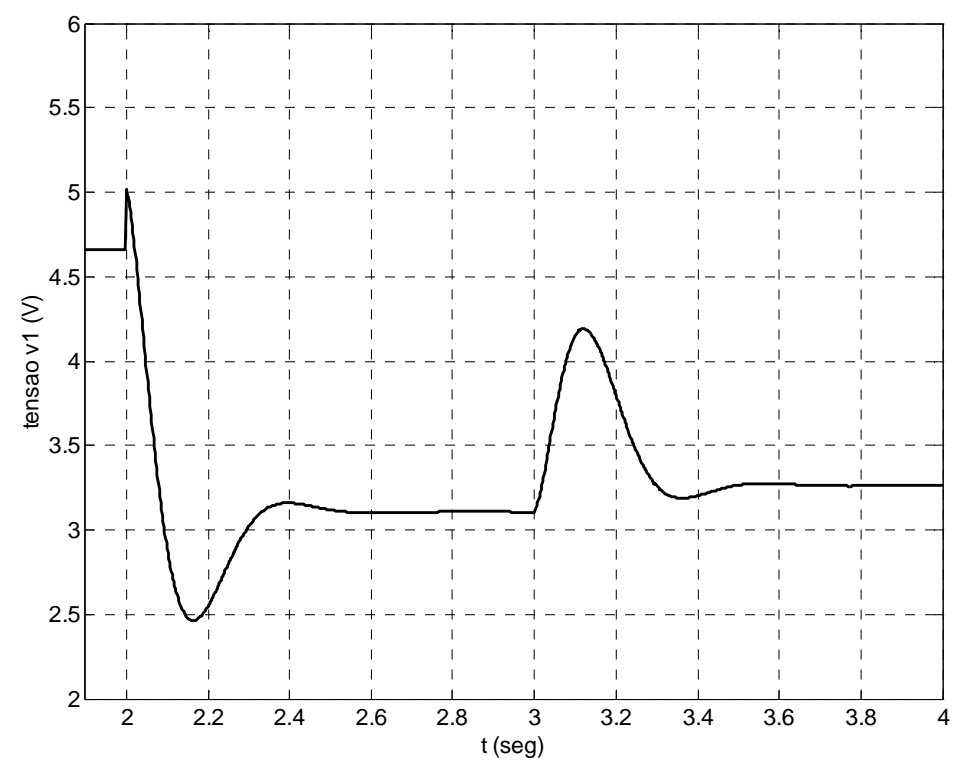

Figura 5.23: Tensão $v_{1}(\mathrm{t})$ no atuador para o controle SISO.

\subsection{Implementação dos controladores}

Os controladores projetados foram implementados em linguagem Pascal e utilizando-se um microcomputador e uma placa de aquisição de dados com conversores $\mathrm{A} / \mathrm{D}$ de 12 bits e $\mathrm{D} / \mathrm{A}$ de 8 bits.

Os controladores foram discretizados através da aproximação de Tustin e com uma freqüência de amostragem de $5000 \mathrm{~Hz}$. 


\section{Capítulo 6}

\section{RESULTADOS E ANÁLISE COMPARATIVA}

Neste capítulo estão apresentados os resultados experimentais obtidos com a aplicação de uma perturbação em degrau em um dos cantos do veículo, e a análise comparativa das técnicas de controle empregadas e dos modelos considerados.

\subsection{Respostas a uma perturbação em degrau}

Sendo o objetivo principal manter o sistema estável e levitando na presença de perturbações, foi aplicada em uma das saídas do protótipo uma perturbação em degrau, no caso foi escolhida a saída 4. Com esta perturbação, a saída 4 passa a ter o seu valor adicionado de $0,6 \mathrm{~mm}$. O valor da perturbação $(0,6 \mathrm{~mm})$, aplicada no entreferro, foi escolhido de forma que o sistema pudesse voltar à posição de equilíbrio sem interromper a levitação do veículo.

As figuras 6.1 a 6.4 apresentam, respectivamente, os resultados para os seguintes controladores e modelos: controle RLQ com modelo de corpo rígido, controle RLQ com modelo de corpo não rígido, controle RLQ com modelo SISO e controle $\mathrm{H}_{2} / \mathrm{H}_{\infty}$ com modelo de corpo rígido.

Os resultados obtidos são apresentados na forma de gráficos, em função do tempo, dos valores das variáveis de saída do veículo (entreferros $z_{1}(\mathrm{t})$ a $z_{4}(\mathrm{t})$ ) e dos modelos matemáticos $(z(t), \phi(t), \theta(t) e \xi(t))$. O tempo de registro dos sinais após a aplicação da perturbação é de $4 s$.

Os valores de referência para os controladores são $z(t)=5 \mathrm{~mm}$ e $\phi(t)=\theta(t)=\xi(t)=0 \mathrm{rad}$. 

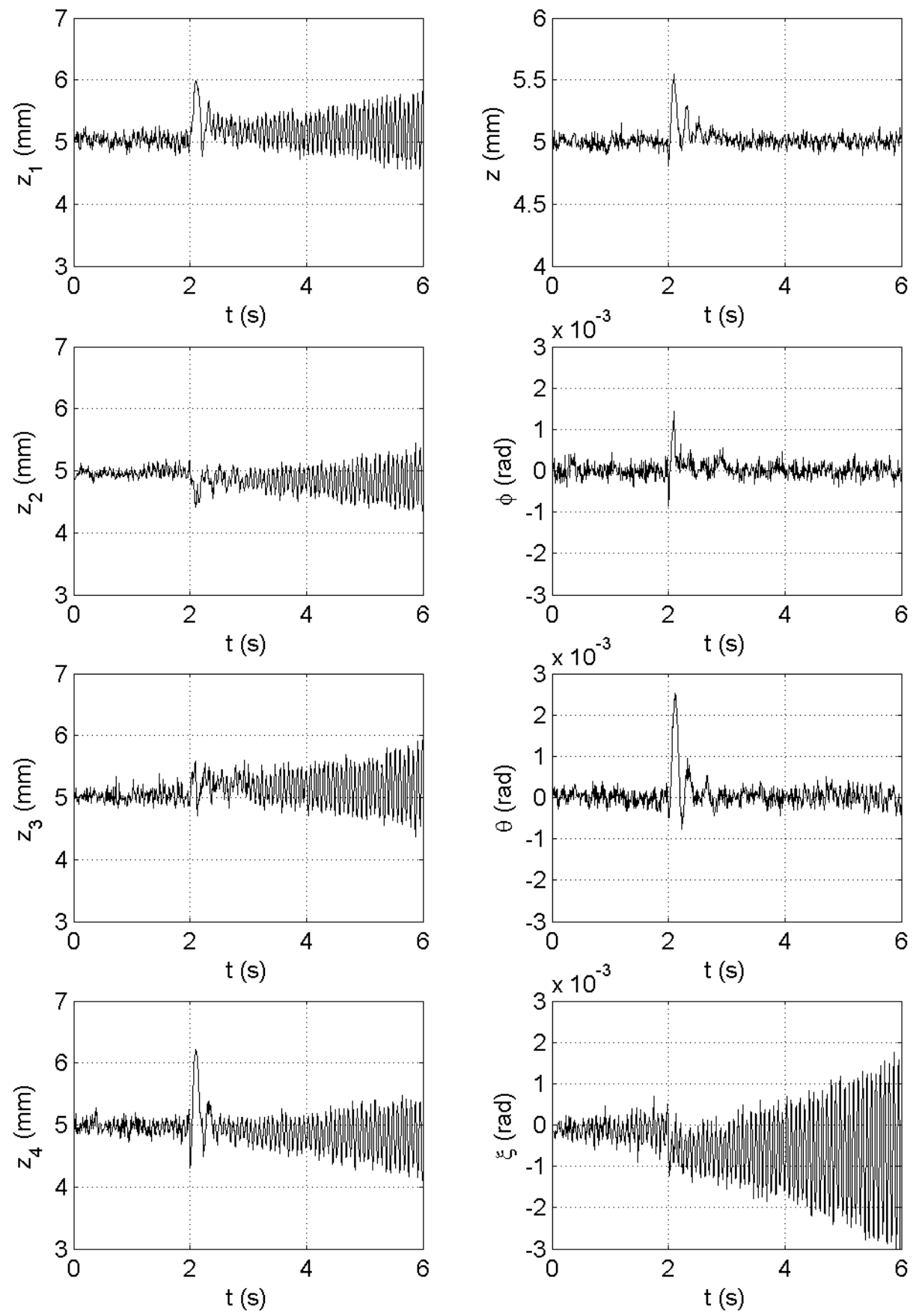

Figura 6.1: Resposta à perturbação em degrau com controle RLQ e modelo de corpo rígido. 

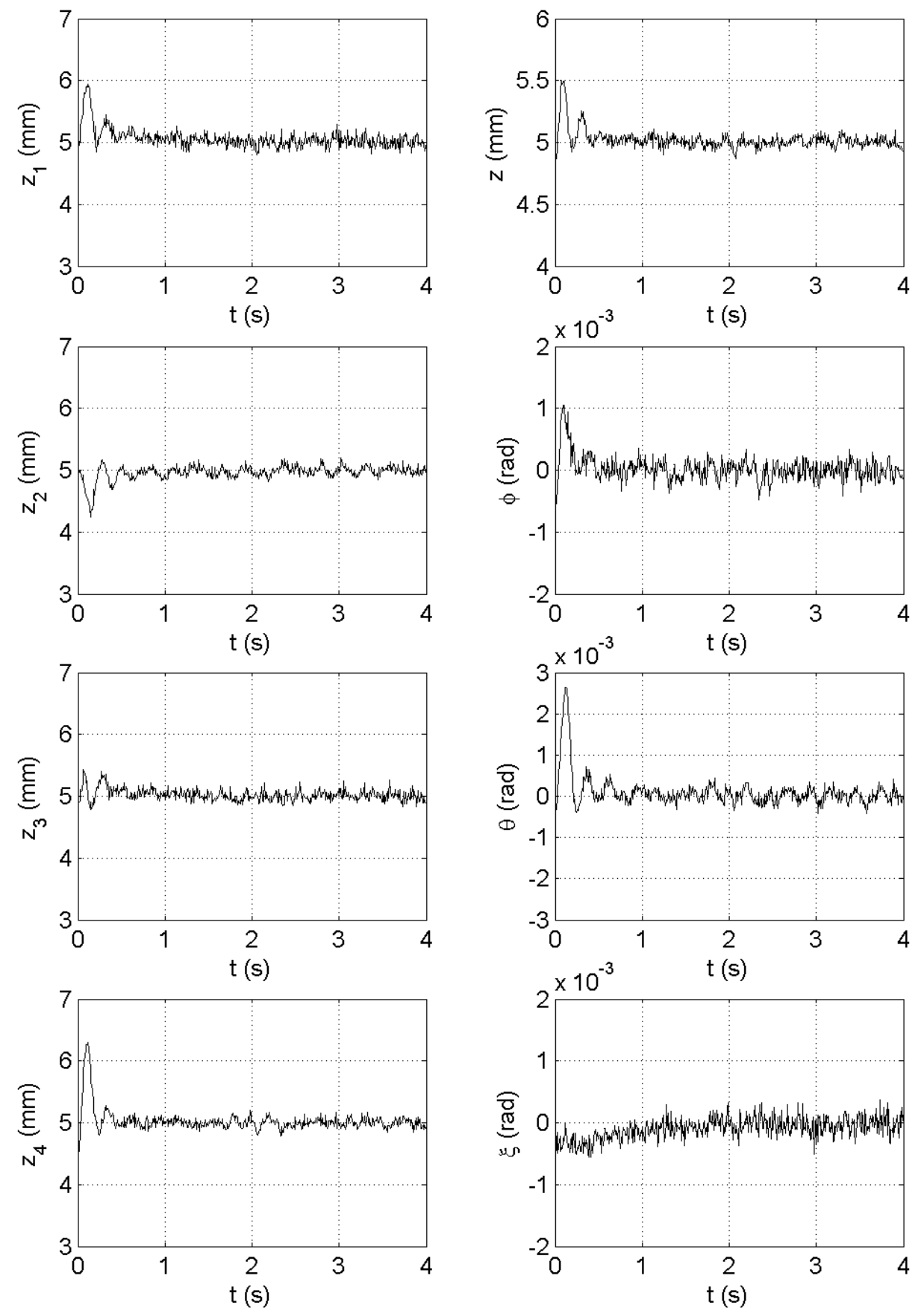

Figura 6.2: Resposta à perturbação em degrau com controle RLQ e modelo de corpo não rígido. 

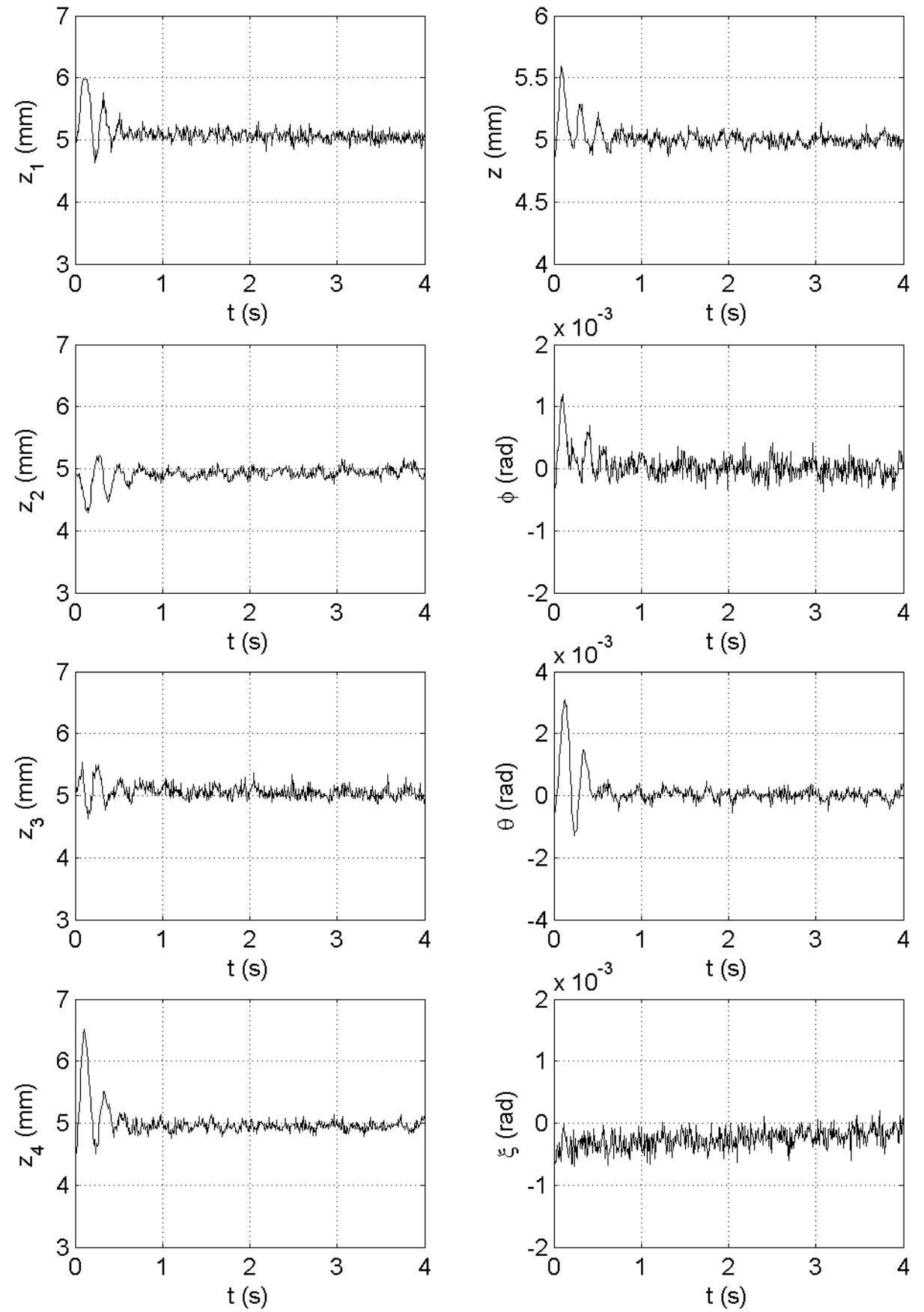

Figura 6.3: Resposta à perturbação em degrau com controle RLQ e modelo SISO. 

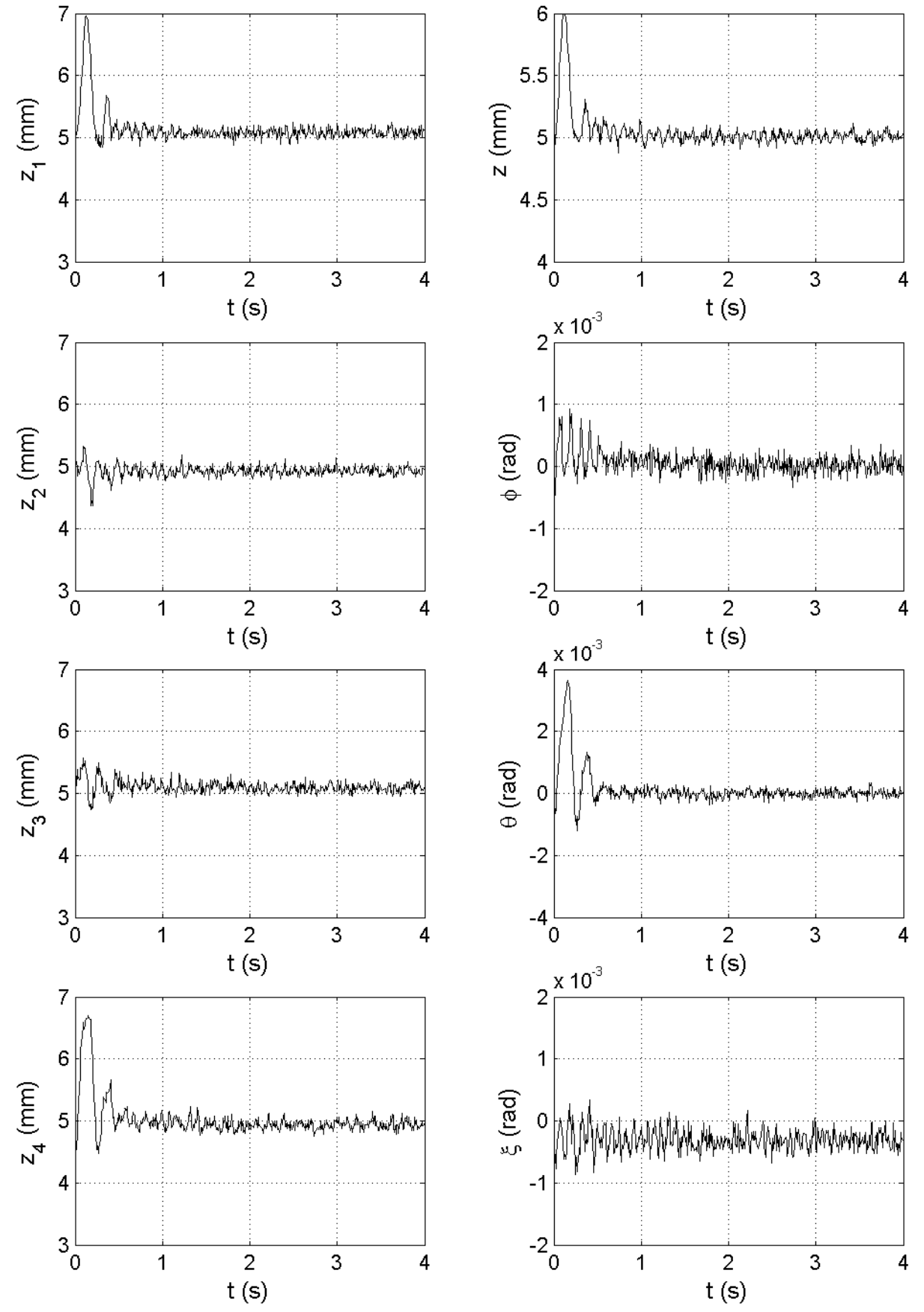

Figura 6.4: Resposta à perturbação em degrau com controle $\mathrm{H}_{2} / \mathrm{H}_{\infty}$ e modelo de corpo rígido. 
Verifica-se, através da figura 6.1, que o sistema com controle RLQ e modelo de corpo rígido é instável, este fato é realçado quando da aplicação da perturbação (no instante de tempo $2 s$ ). As variáveis que são controladas $(z(t), \phi(t) e \theta(t)$ ) permanecem no valor de referência, mas devido à torção observada em $\xi(t)$, que não é uma variável controlada, o sistema torna-se instável, conforme observado em $z_{1}(t)$ a $z_{4}(t)$ e $\xi(t)$.

Diante deste problema foi desenvolvido o controlador RLQ considerando o modelo de corpo não rígido, onde a torção passa a ser uma variável controlada. A figura 6.2 mostra que o sistema permanece estável e a perturbação é totalmente rejeitada no estado estacionário com tempo de acomodação menor do que $0,8 \mathrm{~s}$, conforme especificações de projeto.

O desvio no valor do ângulo de torção, que apareceu com a perturbação, foi eliminado com um tempo de acomodação menor do que $1,5 \mathrm{~s}$, não atendendo à especificação de projeto $(<0,8 s)$. Como comentado, o modelo do sistema para o movimento de torção deveria considerar a elasticidade do veículo.

Da figura 6.3, para o controle RLQ e modelo SISO, onde $z_{1}(t)$ a $z_{4}(t)$ são variáveis controladas, e $z(t), \phi(t), \theta(t) e \xi(t)$ são variáveis indiretamente controladas, tem-se que o sistema é estável e a perturbação é totalmente rejeitada no estado estacionário com tempo de acomodação um pouco maior do que $0,8 \mathrm{~s}$. O desvio no valor do ângulo de torção que apareceu com a perturbação não foi eliminado em $4 s$, mas tende a ser com o tempo.

Verifica-se, da figura 6.4, que o sistema com controle $\mathrm{H}_{2} / \mathrm{H}_{\infty}$ e modelo de corpo rígido é estável e a perturbação é rejeitada no estado estacionário com tempo de acomodação próximo de $0,8 s$ para as variáveis controladas $(z(t), \phi(t) e \theta(t))$. Os valores dos entreferros $\left(z_{1}(t)\right.$ a $\left.z_{4}(t)\right)$ permanecem com um pequeno erro estacionário da ordem de $0,1 \mathrm{~mm}$. O desvio no valor do ângulo de torção que apareceu com a perturbação não foi eliminado em $4 s$ e nem tende a ser com o tempo.

Comparando os controladores, o sistema com controle RLQ e modelo de corpo não rígido apresentou a melhor performance entre todos, pois os menores sobre-sinal e tempo de acomodação foram obtidos com este controlador. O sistema com controle 
RLQ e modelo SISO apresentou resultados intermediários, mas melhor do que o sistema com controle $\mathrm{H}_{2} / \mathrm{H}_{\infty}$ e modelo de corpo rígido, que tem um maior sobresinal, um erro em regime estacionário e não tende a eliminar o erro de torção com o tempo. O sistema com controle RLQ e modelo de corpo rígido é insatisfatório, pois perdeu a estabilidade, apesar de manter sob controle as variáveis controladas $z(t), \phi(t) e \theta(t)$.

\subsection{Análise comparativa}

Para uma análise mais precisa dos resultados, como feito em (BITTAR,1998), foram quantificados, em relação aos valores nominais, os desvios dos valores dos entreferros $z_{1}(t)$ a $z_{4}(t)$ e das variáveis $z(t), \phi(t), \theta(t) e \xi(t)$, através do cálculo de valor quadrático médio ou R.M.S. ("Root Mean Square”) de cada uma das respostas apresentadas nas figuras 6.1 a 6.4 .

O valor R.M.S. é calculado pela seguinte fórmula:

$$
\text { R.M.S. }=\sqrt{\frac{1}{N_{p}} \sum_{k=1}^{N_{p}}\left(x(k)-X_{0}\right)^{2}},
$$

onde:

$x(k)$ é o $k$-ésimo valor medido de $z_{p}(t)(p=1, \ldots, 4), z(t), \phi(t), \theta(t)$ ou $\xi(t)$;

$X_{0}=$ valores nominais de $z_{p}(t)(p=1, \ldots, 4), z(t), \phi(t), \theta(t)$ ou $\xi(t)$

$N_{\mathrm{p}}=500$ é o número total de pontos amostrados.

Na tabela 6.1 têm-se os cálculos obtidos dos valores R.M.S. com cada um dos controladores e modelos utilizados. 
Tabela 6.1: Valores R.M.S. para as respostas à perturbação em degrau.

\begin{tabular}{|c|c|c|c|c|}
\cline { 2 - 5 } \multicolumn{1}{c|}{} & \multicolumn{4}{c|}{ Controle / Modelo } \\
\hline Variável & $\begin{array}{c}\text { RLQ / } \\
\text { Corpo rígido }\end{array}$ & $\begin{array}{c}\text { RLQ / } \\
\text { Corpo não rígido }\end{array}$ & $\begin{array}{c}\text { RLQ / } \\
\text { SISO }\end{array}$ & $\begin{array}{c}\mathbf{H}_{2} / \mathbf{H}_{\infty} / \\
\text { Corpo rígido }\end{array}$ \\
\hline$z_{1}(t)$ & Instável & 0,1710 & 0,2036 & 0,3115 \\
\hline$z_{2}(t)$ & Instável & 0,1227 & 0,1531 & 0,1198 \\
\hline$z_{3}(t)$ & Instável & 0,1009 & 0,1341 & 0,1464 \\
\hline$z_{4}(t)$ & Instável & 0,1952 & 0,2290 & 0,3182 \\
\hline$z(t)$ & 0,0933 & 0,0826 & 0,0910 & 0,1610 \\
\hline$\phi(t)$ & $2,2056.10^{-4}$ & $2,0654.10^{-4}$ & $2,1652.10^{-4}$ & $1,8741.10^{-4}$ \\
\hline$\theta(t)$ & $4,1428 \cdot 10^{-4}$ & $4,1445 \cdot 10^{-4}$ & $5,0936.10^{-4}$ & $5,9219 \cdot 10^{-4}$ \\
\hline$\xi(t)$ & $11,6614 \cdot 10^{-4}$ & $1,9701 \cdot 10^{-4}$ & $3,0258 \cdot 10^{-4}$ & $3,4582.10^{-4}$ \\
\hline
\end{tabular}

Verifica-se, através da tabela 6.1, que o sistema com controle RLQ e modelo de corpo não rígido, assim como na análise gráfica anterior, é o que apresenta o melhor desempenho, ou seja, os menores desvios em relação aos valores nominais.

O sistema com controle RLQ e modelo de corpo rígido apresenta o pior desempenho, mas $z(t), \phi(t) e \theta(t)$ apresentam resultados satisfatórios.

O segundo melhor controle foi o RLQ com modelo SISO e o terceiro, o $\mathrm{H}_{2} / \mathrm{H}_{\infty}$ com modelo de corpo rígido.

Observa-se na tabela 6.1 que:

- os valores R.M.S. para $z_{1}(t)$ e $z_{2}(t)$ são maiores do que os valores para $z_{3}(t)$ e $z_{4}(t)$

- os valores R.M.S da arfagem $(\theta(t))$ para todos os controladores são maiores do que os da rolagem $(\phi(t))$.

Diante do exposto anteriormente e através das figuras 6.1 a 6.4 , percebe-se, que na presença da perturbação o sistema tende mais a executar um movimento de arfagem do que de rolagem. 


\section{Capítulo 7}

\section{CONCLUSÕES}

Este trabalho abordou um tema de grande interesse na área de sistemas de transportes, com o estudo de controladores para a suspensão eletromagnética de um veículo. Está área é promissora e tem grande potencial de desenvolvimento, como demonstra o exemplo da primeira linha de trem comercialmente em operação em Shanghai na China, empregando a tecnologia de levitação por suspensão eletromagnética.

Neste trabalho três modelos matemáticos do sistema foram considerados para o veículo, a saber, um modelo de corpo rígido, um modelo de corpo não rígido e um modelo SISO.

Através da análise dos resultados, verificou-se que o modelo de corpo não rígido é o mais apropriado para representar o sistema, pois no protótipo estão presentes torções e até mesmo flexões. $\mathrm{O}$ modelo de corpo não rígido, utilizado no projeto dos controladores, poderia ser aperfeiçoado para levar em conta também a flexibilidade do veículo. Acredita-se que com isso resultaria melhorada a resposta temporal do sistema quando sujeito a uma perturbação.

O sistema com controle RLQ projetado com base no modelo de corpo não rígido foi aquele que apresentou o melhor desempenho, ou seja, os menores desvios em relação aos valores de referência, quando submetido a uma perturbação em degrau.

O sistema com controle RLQ projetado com base no modelo de corpo rígido apresentou um resultado insatisfatório, sistema instável. Por outro lado, utilizando-se o mesmo modelo para implementar o controlador $\mathrm{H}_{2} / \mathrm{H}_{\infty}$, o sistema tornou-se estável. Considerando que o controlador $\mathrm{H}_{2} / \mathrm{H}_{\infty}$ foi sintetizado a partir de um modelo SISO desacoplado e que foi ajustado por meio de um processo de identificação de sistemas (BITTAR, 1998), este controlador pode estar levando em consideração as características da não rigidez apresentadas pelo veículo. 
Em princípio, os movimentos de rolagem, arfagem e torção não deveriam necessitar de um integrador no controlador, pois os modelos de tais movimentos não estão sujeitos às perturbações de torque. No entanto, na prática este integrador foi necessário para eliminar o erro em regime estacionário causado por imperfeições e assimetrias da distribuição da massa do veículo.

O método para alocação dos pólos de malha fechada do sistema, via lugar das raízes, mostrou-se eficaz como ferramenta de projeto dos controladores RLQ. Para atender os critérios de desempenho, através do lugar das raízes definem-se a matriz $Q$ de peso dos estados e o parâmetro $\rho$ de ponderação do controle de uma maneira mais direcionada, obtendo-se a matriz $K$ de ganhos de realimentação dos estados.

Quando do projeto dos controladores RLQ, existe um conflito entre uma boa resposta a um degrau na referência e a resposta à perturbação. O projeto considerando um pequeno sobre-sinal devido ao degrau na referência leva a um elevado tempo de acomodação devido à perturbação. Neste trabalho, a principal preocupação foi garantir o desempenho do sistema quando sujeito à perturbação. Deste modo, o sistema de controle não foi projetado para conduzir o veículo desde o repouso até a posição de equilíbrio. Para levá-lo até esta posição, na partida do sistema, pode-se escolher um dos "zeros auxiliares" de malha aberta próximo da origem. Desta maneira, quando se aumenta o ganho $(1 / \rho)$, um dos pólos de malha fechada tende a cancelar o zero de malha fechada e o sistema é regido apenas por dois pólos complexos conjugados. Com isto, pode-se conseguir um bom desempenho na partida do sistema, com um pequeno sobre-sinal. Então, decorrido um certo tempo para que o sistema atinja o equilíbrio, pode-se transferir o comando para o controlador projetado neste trabalho.

Não foi necessária a implementação de filtro passa-baixas para atenuação do ruído de altas freqüências, presente no sinal de saída do sensor de posição.

Os acelerômetros, utilizados neste trabalho, são mais apropriados para a medida de vibrações. A sua utilização para obtenção das medidas de velocidade foi um processo que exigiu uma longa pesquisa e avaliação de diversas alternativas para resolver o problema da deriva. Chegou-se, por fim, à solução empregando um filtro passa-altas antes do integrador utilizado para o cálculo da velocidade. 
Outras dificuldades identificadas durante o projeto e implementação do sistema foram:

- a calibração do sensor de posição devido a irregularidades do entreferro e o fato do sensor de posição medir indiretamente a distância do entreferro, não detectando irregularidades do trilho;

- a determinação da relação força/tensão que depende de uma calibração precisa do sensor de posição;

- a presença de torção e flexão no veículo, tendo sido acrescentadas barras transversais com o objetivo de reduzir o problema e tornar o protótipo mais rígido;

A seguir são apresentadas algumas sugestões de pesquisas futuras para efeito de comparação e melhoria do sistema:

- utilização do acelerômetro, ao invés do sensor indutivo, para estimar a medida de posição;

- instalação de acelerômetro com dois eixos de sensibilidade para medir diretamente os ângulos de rolagem e arfagem;

- projetos de circuitos que melhorem o desempenho do acelerômetro na estimação da velocidade, diminuindo a influência do erro de off-set e deriva térmica existente; utilização de outros filtros com um maior número de pólos ou com largura de banda diferente, e até mesmo a construção de circuitos analógicos;

- melhoria na modelagem matemática, considerando torções e flexões do veículo ou, talvez, até uma análise por elementos finitos;

- utilização do controle robusto baseado no procedimento LQG/LTR ('Linear Quadratic Gaussian / Loop Transfer Recovery"). Sendo conhecida a matriz de ganhos de realimentação de estados $(K)$, obtida neste trabalho, está poderia ser utilizada no procedimento de recuperação, considerando a abertura da malha na entrada da planta (procedimento dual daquele apresentado em (CRUZ, 1996)) - neste caso seria utilizada uma realimentação de saída em lugar de uma realimentação de estados, como é o caso do RLQ;

- desenvolvimento de controladores baseados na técnica de controle nebuloso (controle Fuzzy) ou utilizando redes neurais. 


\section{ANEXOS}

\section{ANEXO A - CIRCUITO REGULADOR DE VOLTAGEM}

Como informado, sendo a tensão de saída diretamente proporcional à tensão de alimentação, o acelerômetro requer uma tensão estabilizada. Para isto foi desenvolvido um circuito regulador de voltagem que fornece uma tensão constante de $5 V \mathrm{cc}$ para alimentação do acelerômetro $\left(V_{\mathrm{S}}\right)$. O regulador de voltagem, apresentado na figura A.1, foi implementado com o circuito integrado LM723 e aproveitou-se da tensão de $17 \mathrm{Vcc}$ existente no protótipo do veículo.

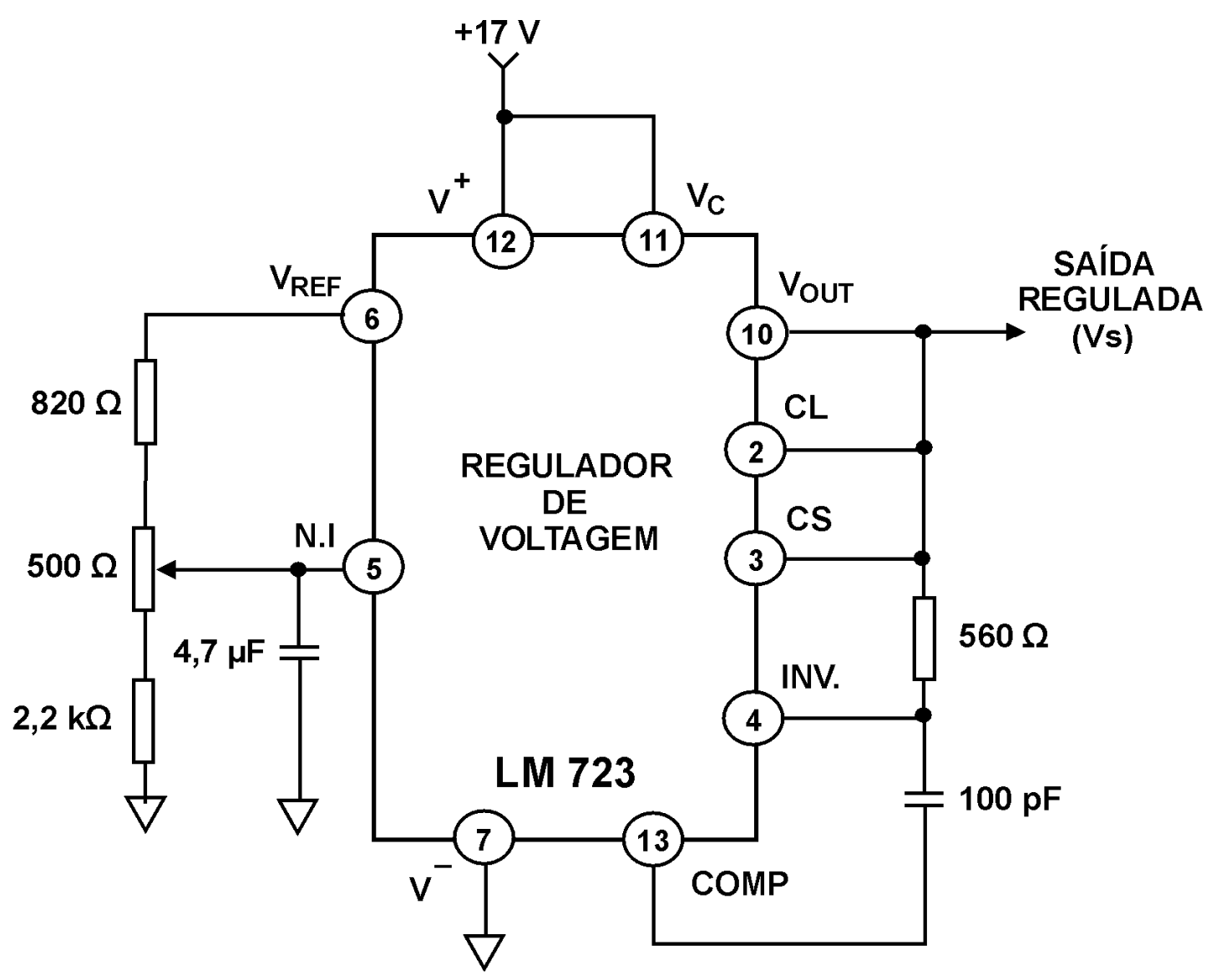

Figura A.1: Circuito regulador de voltagem. 


\section{ANEXO B - CALIBRAÇÃO DOS SENSORES DE POSIÇÃO}

As distâncias dos entreferros $z_{1}, z_{2}, z_{3}$ e $z_{4}$ são medidas pelos sensores de posição do tipo indutivo.

Conforme apresentado em (BITTAR,1998), a equação linear levantada experimentalmente, que descreve a conversão da leitura do sinal do sensor de uma variável em milímetros para uma variável em Volts, é dada por:

$$
z_{m m}=k_{s} z_{v}-1,3
$$

onde: $z_{\mathrm{mm}}$ e $z_{\mathrm{v}}$ são os entreferros medidos, respectivamente, em milímetros e Volts, com

$$
k_{s}=0,9\left(\frac{m m}{V}\right) .
$$

Neste trabalho, novas equações foram levantadas experimentalmente e agora individualmente para cada sensor de posição.

O procedimento adotado está descrito a seguir.

Espaçadores de espessuras variadas foram colocados em cada um dos quatro entreferros e, com um multímetro, foram medidas as tensões dos entreferros em Volts. Obteve-se assim a tabela B.1.

Os dados da tabela B.1 foram aproximados por uma reta, através da utilização do método dos mínimos quadrados.

As novas equações das retas são:

$$
\begin{aligned}
& \text { sensor } 1: z_{m m}=0,83 z_{v}-0,2 ; \\
& \text { sensor 2: } z_{m m}=0,79 z_{v}-1,3 ; \\
& \text { sensor 3: } z_{m m}=0,83 z_{v}+0,0 ; \\
& \text { sensor } 4: z_{m m}=0,83 z_{v}+0,1
\end{aligned}
$$


Tabela B.1.: Dados dos entreferros em Volts e milímetros.

\begin{tabular}{|c|c|c|c|c|}
\hline Entreferro (mm) & Sensor $\mathbf{1}(\mathbf{V})$ & Sensor 2 (V) & Sensor 3 (V) & Sensor 4 (V) \\
\hline $\mathbf{2 , 1 0}$ & 2,84 & 3,52 & 2,65 & 2,45 \\
\hline $\mathbf{2 , 1 0}$ & 2,79 & 3,64 & 2,58 & 2,52 \\
\hline $\mathbf{2 , 1 0}$ & 2,86 & 3,63 & 2,57 & 2,37 \\
\hline $\mathbf{2 , 1 0}$ & 2,78 & 3,72 & 2,54 & 2,34 \\
\hline $\mathbf{3 , 6 0}$ & 4,47 & 6,62 & 4,01 & 3,91 \\
\hline $\mathbf{3 , 6 0}$ & 4,36 & 6,57 & 3,98 & 3,95 \\
\hline $\mathbf{3 , 6 0}$ & 4,39 & 6,55 & 3,90 & 3,92 \\
\hline $\mathbf{3 , 6 0}$ & 4,28 & 6,63 & 3,76 & 4,07 \\
\hline $\mathbf{4 , 7 5}$ & 5,86 & 8,22 & 5,79 & 6,01 \\
\hline $\mathbf{4 , 7 5}$ & 5,75 & 8,34 & 5,69 & 5,97 \\
\hline $\mathbf{4 , 7 5}$ & 5,96 & 8,31 & 5,87 & 5,84 \\
\hline $\mathbf{4 , 7 5}$ & 5,85 & 8,33 & 5,56 & 5,91 \\
\hline $\mathbf{6 , 4 0}$ & 7,97 & 10,04 & 7,97 & 7,92 \\
\hline $\mathbf{6 , 4 0}$ & 8,13 & 9,98 & 7,81 & 7,97 \\
\hline $\mathbf{6 , 4 0}$ & 8,12 & 10,01 & 8,05 & 7,92 \\
\hline $\mathbf{6 , 4 0}$ & 8,08 & 10,04 & 8,18 & 7,91 \\
\hline $\mathbf{8 , 5 0}$ & 10,32 & 11,44 & 9,83 & 9,82 \\
\hline $\mathbf{8 , 5 0}$ & 10,21 & 11,42 & 9,94 & 10,03 \\
\hline $\mathbf{8 , 5 0}$ & 10,41 & 11,39 & 9,88 & 9,81 \\
\hline $\mathbf{8 , 5 0}$ & 10,29 & 11,41 & 9,71 & 9,83 \\
\hline
\end{tabular}

Não foram utilizados filtros para atenuação de ruídos de altas freqüências. 


\section{ANEXO C - CALIBRAÇÃO DOS ATUADORES}

Sabe-se de (BITTAR,1998) que a força de atração do eletroímã é dada por:

$$
F(t)=k_{f}\left(\frac{i(t)}{z(t)}\right)^{2}
$$

onde a constante proporcional $k_{f}$ é:

$$
k_{f}=\frac{\mu_{0} a c N^{2}}{2} .
$$

Das equações (C.1) e (C.2) têm-se que:

$i(\mathrm{t})$ : corrente elétrica na bobina;

$z(\mathrm{t})$ : distância do entreferro;

$\mu_{0}:$ permeabilidade do ar $\left(\mu_{0}=4 \pi 10^{-7}(\mathrm{H} / \mathrm{m})\right)$;

$a, c:$ dimensões do eletroímã $(a=0,03 m ; c=0,064 m)$;

$N$ : numero de espiras $(N=1300)$.

Considerando que para regiões próximas do ponto de equilíbrio, com o veículo no estado de levitação, o ganho do circuito de potência $k_{\mathrm{pot}}$ é aproximadamente constante, então vale que:

$$
v(t)=k_{p o t} i(t)
$$

onde:

$v(\mathrm{t})$ : tensão aplicada na entrada do circuito de potência;

$k_{\text {pot }}=1,68$.

Substituindo a equação (C.3) em (C.1), pode-se escrever a força de atração eletromagnética em função da tensão aplicada na entrada do circuito de potência $(v(t))$, ao invés da corrente na bobina, então: 


$$
F(t)=k_{v}\left(\frac{v(t)}{z(t)}\right)^{2}
$$

sendo que a constante proporcional $k_{v}$ para tensão aplicada é dada por:

$$
k_{v}=\frac{k_{f}}{k_{p o t}^{2}} .
$$

O valor teórico da constante $k_{\mathrm{v}}$, no ponto de equilíbrio, é dado por:

$$
k_{v}=7,22 * 10^{-4}\left(\frac{\mathrm{Nm}^{2}}{V^{2}}\right) .
$$

As constantes $k_{v i}(i=1, \ldots, 4)$ para cada eletroímã também foram levantadas experimentalmente. O veículo foi colocado em levitação, utilizando o controlador apresentado em (COSTA et al., 2003). Massas de diferentes valores foram colocadas sobre o veículo e medidas, utilizando-se um multímetro, as correspondentes tensões, fornecidas ao circuito de potência ou atuador, conforme tabela C.1.

Tabela C.1.: Dados da relação força aplicada por tensão em cada atuador.

\begin{tabular}{|c|c|c|c|c|c|c|}
\hline Massa Total (kg) & Força Total (N) & Força 1,2,3,4 (N) & Atuador 1 (V) & Atuador 2 (V) & Atuador 3 (V) & Atuador 4 (V) \\
\hline $\mathbf{8 8 , 3 0}$ & 865,96 & 216,49 & 3,31 & 2,78 & 3,18 & 3,14 \\
\hline $\mathbf{8 8 , 3 0}$ & 865,96 & 216,49 & 3,30 & 2,76 & 3,17 & 3,13 \\
\hline $\mathbf{8 8 , 3 0}$ & 865,96 & 216,49 & 3,32 & 2,76 & 3,17 & 3,13 \\
\hline $\mathbf{8 8 , 3 0}$ & 865,96 & 216,49 & 3,32 & 2,77 & 3,16 & 3,12 \\
\hline $\mathbf{9 2 , 5 0}$ & 907,15 & 226,79 & 3,40 & 2,84 & 3,27 & 3,22 \\
\hline $\mathbf{9 2 , 5 0}$ & 907,15 & 226,79 & 3,39 & 2,82 & 3,25 & 3,21 \\
\hline $\mathbf{9 2 , 5 0}$ & 907,15 & 226,79 & 3,39 & 2,84 & 3,29 & 3,21 \\
\hline $\mathbf{9 2 , 5 0}$ & 907,15 & 226,79 & 3,39 & 2,84 & 3,27 & 3,22 \\
\hline $\mathbf{9 6 , 7 0}$ & 948,34 & 237,08 & 3,45 & 2,89 & 3,34 & 3,29 \\
\hline $\mathbf{9 6 , 7 0}$ & 948,34 & 237,08 & 3,43 & 2,92 & 3,31 & 3,27 \\
\hline $\mathbf{9 6 , 7 0}$ & 948,34 & 237,08 & 3,45 & 2,91 & 3,35 & 3,28 \\
\hline $\mathbf{9 6 , 7 0}$ & 948,34 & 237,08 & 3,46 & 2,89 & 3,36 & 3,28 \\
\hline $\mathbf{1 0 0 , 9 0}$ & 989,53 & 247,38 & 3,52 & 2,96 & 3,41 & 3,36 \\
\hline $\mathbf{1 0 0 , 9 0}$ & 989,53 & 247,38 & 3,52 & 3,02 & 3,42 & 3,36 \\
\hline $\mathbf{1 0 0 , 9 0}$ & 989,53 & 247,38 & 3,52 & 3,01 & 3,42 & 3,35 \\
\hline $\mathbf{1 0 0 , 9 0}$ & 989,53 & 247,38 & 3,51 & 2,97 & 3,41 & 3,35 \\
\hline
\end{tabular}


As tensões dos sensores de posição também foram medidas, e calculados (equações (B.3)-(B.6)) os respectivos valores de entreferro em regime estacionário, tabela C.2.

Tabela C.2.: Valores de entreferro em Volts e milímetros.

\begin{tabular}{|c|c|c|}
\hline $\begin{array}{c}\text { Sensor } / \\
\text { Entreferro }\end{array}$ & $\begin{array}{c}z_{\mathrm{v}} \\
(V)\end{array}$ & $\begin{array}{c}z_{m m} \\
(\mathrm{~mm})\end{array}$ \\
\hline 1 & 6,90 & 5,53 \\
\hline 2 & 7,90 & 4,94 \\
\hline 3 & 6,90 & 5,73 \\
\hline 4 & 7,10 & 5,99 \\
\hline
\end{tabular}

Utilizando-se dos dados das tabelas C.1 e C.2, e da equação (C.4), para cada valor de força e tensão, obteve-se as constantes $k_{\mathrm{vi}}$ e no final foi calculada a média aritmética das constantes encontradas para cada eletroímã, gerando a tabela C.4.

Um segundo método foi utilizado no cálculo das constantes $k_{\mathrm{vi}}$, conforme descrito a seguir. Este método não necessita do valor da massa do veículo, apenas do valor da massa colocada sobre o veículo.

Assume-se na situação em que o veículo está levitando em regime estacionário e sem carga, que $F_{0}, Z_{0}$ e $V_{0}$ são respectivamente: força vertical de atração, valor de entreferro e tensão do atuador.

Colocando-se uma massa conhecida sobre o veículo, os novos valores de força de atração $(F)$, de entreferro $(\mathrm{z})$ e tensão do atuador $(v)$ são dados por:

$$
F=F_{0}+\Delta F ; \quad z=Z_{0} ; \quad v=V_{0}+\Delta V
$$

Substituindo a equação (C.7) em (C.4), tem-se que:

$$
F_{0}+\Delta F=k_{v}\left(\frac{V_{o}+\Delta V}{Z_{0}}\right)^{2}
$$


Sabe-se que:

$$
F_{0}=k_{v}\left(\frac{V_{0}}{Z_{0}}\right)^{2} \text {. }
$$

Substituindo a equação (C.9) em (C.8) tem-se que:

$$
k_{v}=\frac{Z_{0}^{2} \Delta F}{2 V_{0} \Delta V+\Delta V^{2}} .
$$

Utilizando-se dos dados da tabela C.3 e da equação (C.10), obtêm-se as constantes $k_{\mathrm{vi}}$ para cada eletroímã, conforme tabela C.4.

Tabela C.3.: Parâmetros para cálculo de $k_{\mathrm{vi}}$.

\begin{tabular}{|c|c|c|c|c|}
\hline Parâmetros & $\boldsymbol{k}_{\mathrm{v} \mathbf{1}}$ & $\boldsymbol{k}_{\mathrm{v} \mathbf{2}}$ & $\boldsymbol{k}_{\mathbf{v} 3}$ & $\boldsymbol{k}_{\mathbf{v} 4}$ \\
\hline$\Delta \boldsymbol{F}(\boldsymbol{N})$ & 30,89 & 30,89 & 30,89 & 30,89 \\
\hline $\boldsymbol{V}_{\mathbf{0}}(\boldsymbol{V})$ & 3,31 & 2,77 & 3,17 & 3,13 \\
\hline$\Delta \boldsymbol{V}(\boldsymbol{V})$ & 0,21 & 0,22 & 0,25 & 0,23 \\
\hline $\boldsymbol{Z}_{\mathbf{0}}(\boldsymbol{m m})$ & 5,53 & 4,94 & 5,73 & 5,99 \\
\hline
\end{tabular}

Quando da utilização das constantes $k_{\mathrm{vi}}$ obtidas anteriormente, foi observado que o veiculo em levitação apresentava um pequeno deslocamento em relação ao movimento de guinada ("yaw"). Para amenizar este efeito, os valores de $k_{\mathrm{vi}}$ finais foram ajustados na prática. Estes valores são apresentados na tabela C.4. 
Tabela C.4.: Constantes $k_{\mathrm{vi}}$ para os eletroímãs.

\begin{tabular}{|c|c|c|c|}
\hline $\begin{array}{c}\text { Eletroímã / } \\
\text { Constante } \\
k_{\mathrm{vi}}\end{array}$ & $\begin{array}{c}k_{\mathrm{vi}} \text { obtida pela } \\
\text { equação C.4 } \\
{\left[\frac{\mathrm{Nm}^{2}}{V^{2}}\right]}\end{array}$ & $\begin{array}{c}k_{\mathrm{vi}} \text { obtida pela } \\
\text { equação C.10 } \\
\left.\qquad \frac{\mathrm{Nm}^{2}}{V^{2}}\right]\end{array}$ & $\begin{array}{c}\text { Valores ajustados } \\
\boldsymbol{k}_{\mathbf{v i}} \\
{\left[\frac{N m^{2}}{V^{2}}\right]}\end{array}$ \\
\hline $1\left(k_{\mathrm{v} 1}\right)$ & 0,000606 & 0,000659 & 0,000600 \\
\hline $2\left(k_{\mathrm{v} 2}\right)$ & 0,000685 & 0,000595 & 0,000720 \\
\hline $3\left(k_{\mathrm{v} 3}\right)$ & 0,000698 & 0,000616 & 0,000610 \\
\hline $4\left(k_{\mathrm{v} 4}\right)$ & 0,000791 & 0,000743 & 0,000780 \\
\hline
\end{tabular}

Percebe-se que os dois métodos experimentais para o cálculo das constantes $k_{\mathrm{vi}}$ dependem de uma calibração precisa dos sensores de posição (anexo B). 


\section{ANEXO D - PROGRAMA DE CONTROLE}

A título de exemplo é apresentado o programa desenvolvido em Pascal para o controle RLQ com modelo de corpo não rígido.

\{RLQ para o Modelo de Corpo nao Rigido com integrador\}

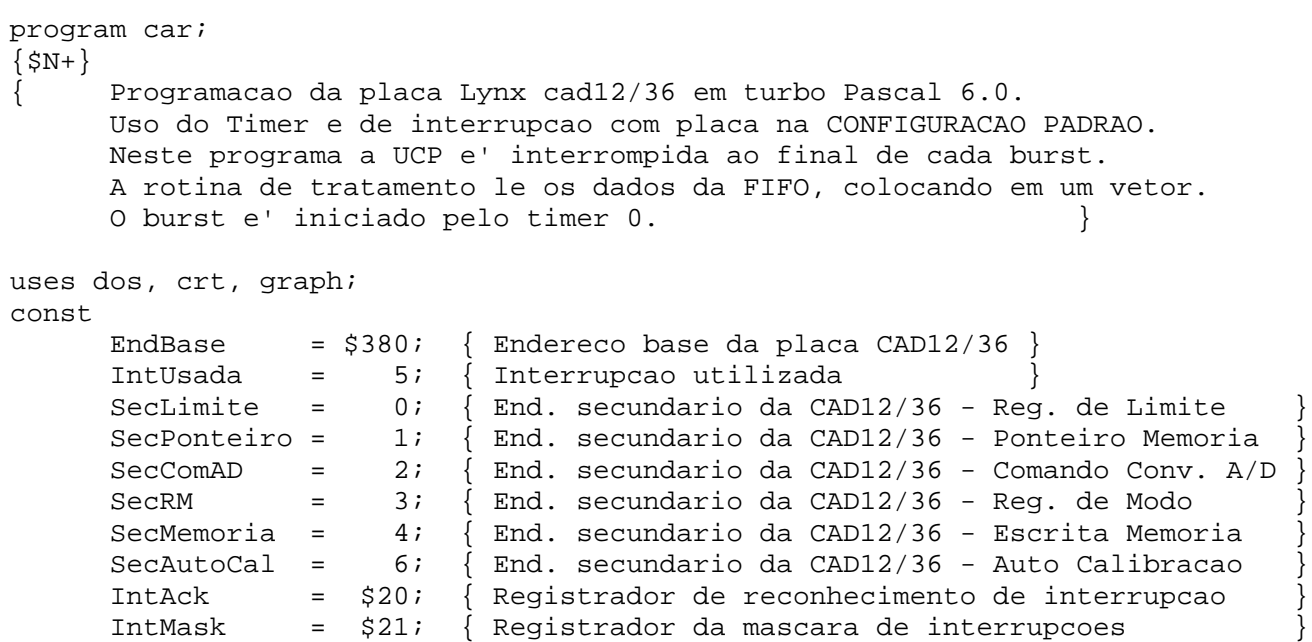




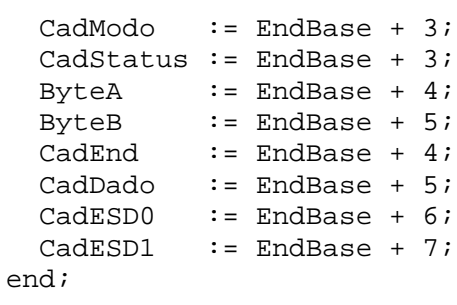

Procedure EscreveRegSecundario (EndReg, Dado: byte);

begin

Port [CadEnd] := EndReg;

Port [CadDado] := Dado;

end;

Procedure EscreveMemCanais (Posicao, Dado: byte);

$\{$ Posicao $=$ ordem de leitura $\}$

\{ Dado = codigo do ganho e o numero do canal A/D \}

begin

EscreveRegSecundario (SecPonteiro, Posicao); \{ Endereco da Memoria de Canais \}

EscreveRegSecundario (SecMemoria, (Dado)); \{ Canal e Ganho correspondente end;

Procedure AutoCalibracao;

begin

EscreveRegSecundario (SecAutocal, $\odot$ );

for $i:=1$ to 4 do

Delay (20000);

end;

Procedure LimpaFIFo;

Var i, Dado: integer;

begin

EscreveRegSecundario $(5,0) ; \quad\{$ comando para limpar a FIF0 $\}$

for $i:=1$ to 16 do

begin

Dado := Port $[$ ByteB]

Dado := Port $[$ ByteA];

end;

Procedure ProgramaTimer (Contador, Modo: byte; Valor: word);

Var EndCtr: word;

begin

EndCtr := EndBase + Contador;

Port [CadModo] := Contador * $64+48+$ Modo * 2 ;

Port [EndCtr] := Lo (Valor):

Port [EndCtr] := Hi (Valor); end;

Procedure InibeINT;

Var Mask : byte;

begin

Mask := Port [IntMask];

Port [IntMask] := Mask or (\$01 shl IntUsada);

end;

Procedure HabilitaINT;

Var Mask : byte;

begin

Mask := Port [IntMask];

Port [IntMask] := Mask and not (\$01 shl IntUsada);

Port [IntAck] :=\$60 + IntUsada; \{ limpa reg. de interrupcoes $\}$ end;

Procedure InibeGateComINT;

begin

EscreveRegSecundario (SecRM, \$๑c);

$\begin{array}{ll}\{\text { inibe timer } & \} \\ \{\text { usa timer } \odot & \} \\ \{\text { interrupcao ao final do burst } & \} \\ \{\text { modo burst ligado } & \} \\ \text { modo DMA desligado } & \}\end{array}$


end;

Procedure HabilitaGateComINT; begin EscreveRegSecundario (SecRM, \$4c);

$\begin{array}{ll}\{\text { habilita timer } & \text { \} } \\ \{\text { usa timer } 0 & \\ \{\text { interrupcao ao final do burst } \\ \{\text { modo burst ligado } \\ \{\text { modo DMA desligado }\end{array}$

end ;

Procedure ProgramaMemoriaCanais; Const

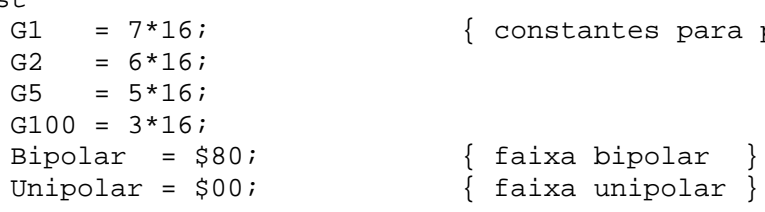


ZO,

yAk, yBk, yCk, yDk, yAk_1, yBk_1,yCk_1, yDk_1,

$y A, y B, y C, y D, y A \_1, y B \_1, y C \_1, y D \_1$,

acel_A, acel_B, acel_C, acel_D, cte_aA, cte_aB, cte_ac, cte_aD,

$\mathrm{VA}, \mathrm{VB}, \mathrm{VC}, \mathrm{VD}, \mathrm{VA} \_1, \mathrm{VB} \_1, \mathrm{VC} \_1, \mathrm{VD} \_1$,

af, bf, cf : extended;

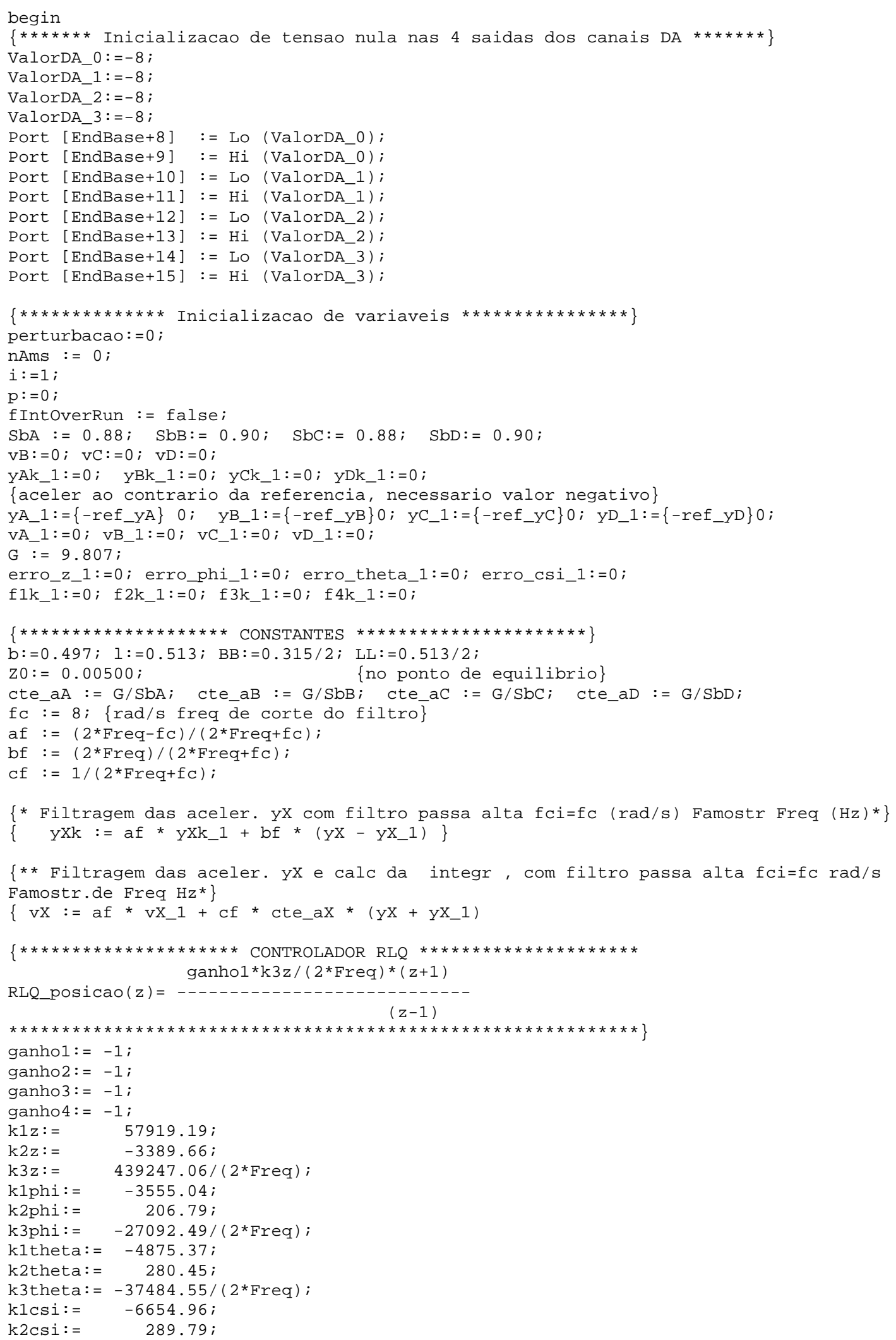




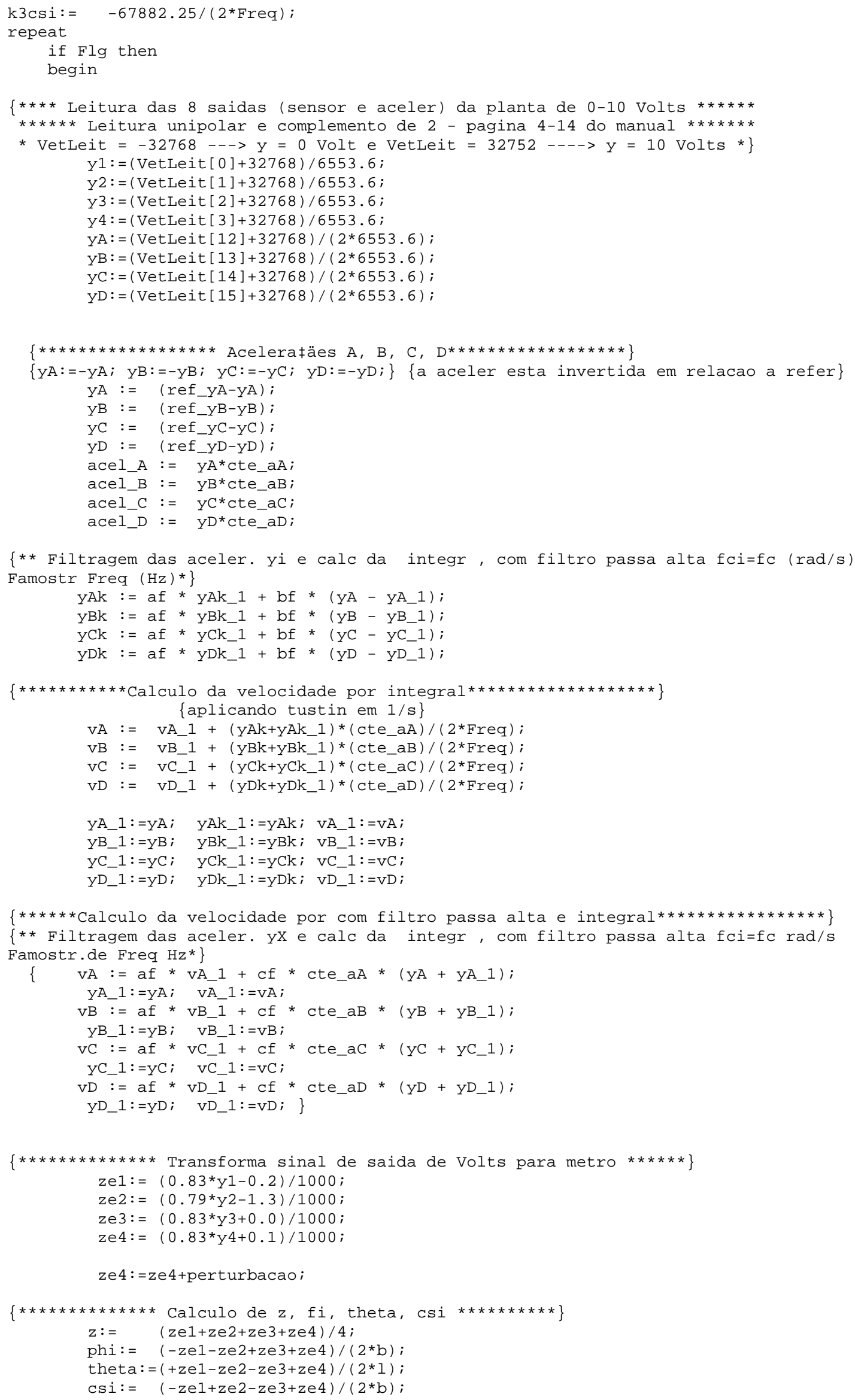

\{** Filtragem das aceler. yi e calc da integr, com filtro passa alta fci=fc ( $\mathrm{rad} / \mathrm{s})$ Famostr Freq $\left.(\mathrm{Hz})^{*}\right\}$

$\left\{* * * * *\right.$ Calculo da velocidade por com filtro passa alta e integral $\left.{ }^{* * * * * * \star * \star * * * \star * * *}\right\}$ $\left\{{ }^{* *}\right.$ Filtragem das aceler. $\mathrm{YX}$ e calc da integr, com filtro passa alta $\mathrm{fci}=\mathrm{fc} \mathrm{rad} / \mathrm{s}$ Famostr. de Freq $\left.\mathrm{Hz}^{*}\right\}$

$\left\{\quad V A:=a f * V A \_1+c f * c t e \_a A *\left(y A+y A \_1\right)\right.$;

YA 1 : $=y A ; \quad$ VA_1:=vA;

$V B:=a f$ * $V B \_1+c f$ * $c$ cte_aB * $\left(y B+y B \_1\right) ;$

yB_1:=yB; VB_1:=vB;

$v C:=a f$ * $v C \_1+c f$ * $c t e \_a C$ * $\left(y C+y C \_1\right) ;$

$\mathrm{yC} \_1:=\mathrm{yC} ; \quad \mathrm{VC} \_1:=\mathrm{vC}$;

$\mathrm{VD}:=\mathrm{af} * \mathrm{VD} \_1+\mathrm{cf} * \mathrm{cte} \_\mathrm{aD} *\left(\mathrm{yD}+\mathrm{yD} \_1\right) ;$

$\left.\mathrm{yD} \_1:=\mathrm{yD} ; \mathrm{VD} \_1:=\mathrm{vD} ;\right\}$

$\{* * * * * * * * * * * * *$ Transforma sinal de saida de Volts para metro $* * * * * *\}$

ze1: $=\left(0.83^{*} y 1-0.2\right) / 1000$

$z e 2:=\left(0.79^{*} y 2-1.3\right) / 1000$

$z e 3:=\left(0.83^{*} y 3+0.0\right) / 1000 ;$

ze $4:=\left(0.83^{*} y 4+0.1\right) / 1000$;

ze4:=ze4+perturbacao;

$\{* * * * * * * * * * * *$ Calculo de $\mathrm{z}, \mathrm{fi}$, theta, csi $* * * * * * * * *\}$

$z:=\quad(z e 1+z e 2+z e 3+z e 4) / 4$;

phi:= $(-z e 1-z e 2+z e 3+z e 4) /\left(2^{*} b\right)$;

theta: $=(+$ ze $1-z e 2-z e 3+z e 4) /(2 * 1)$;

csi: $=(-z e 1+z e 2-z e 3+z e 4) /\left(2^{*} b\right)$ 


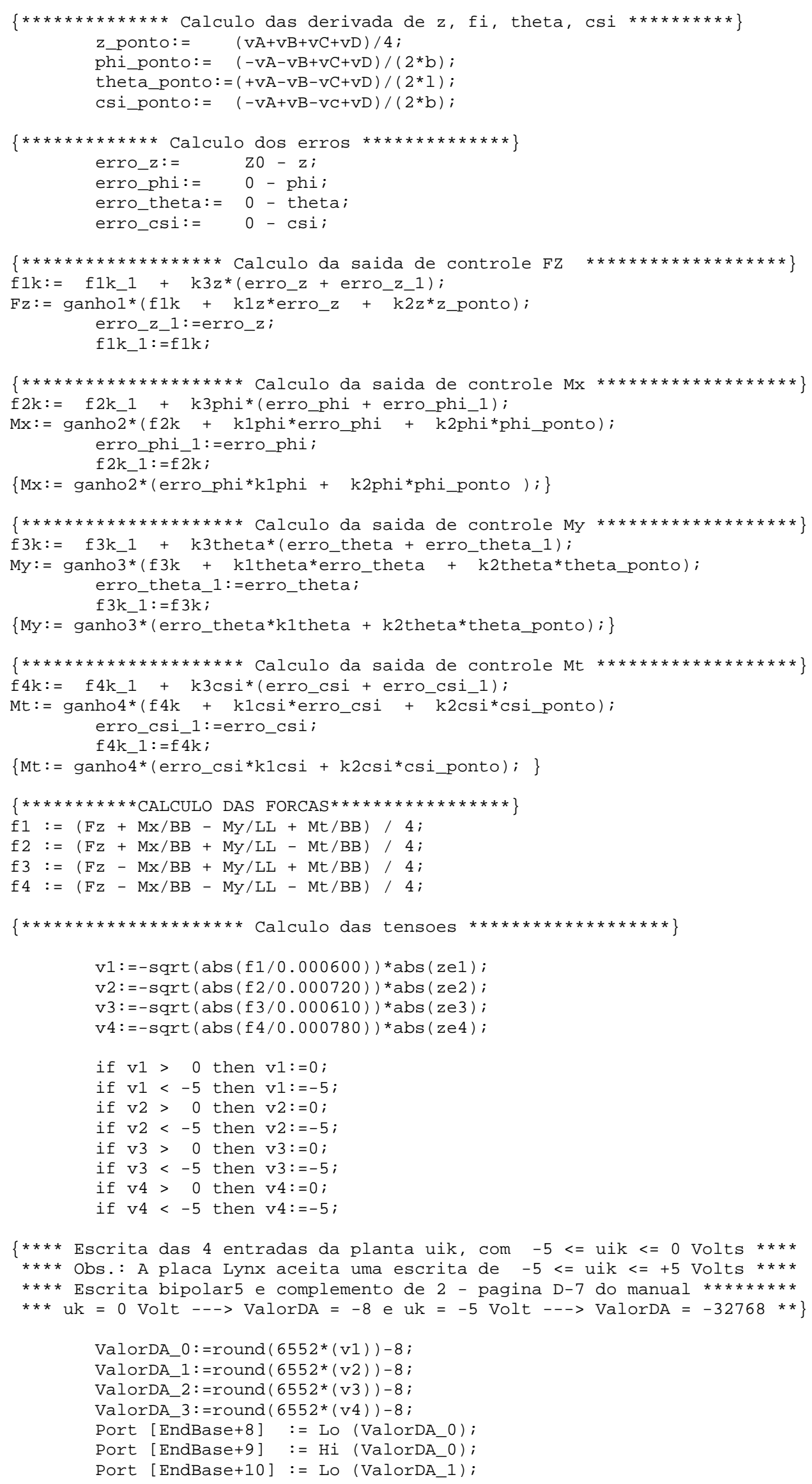




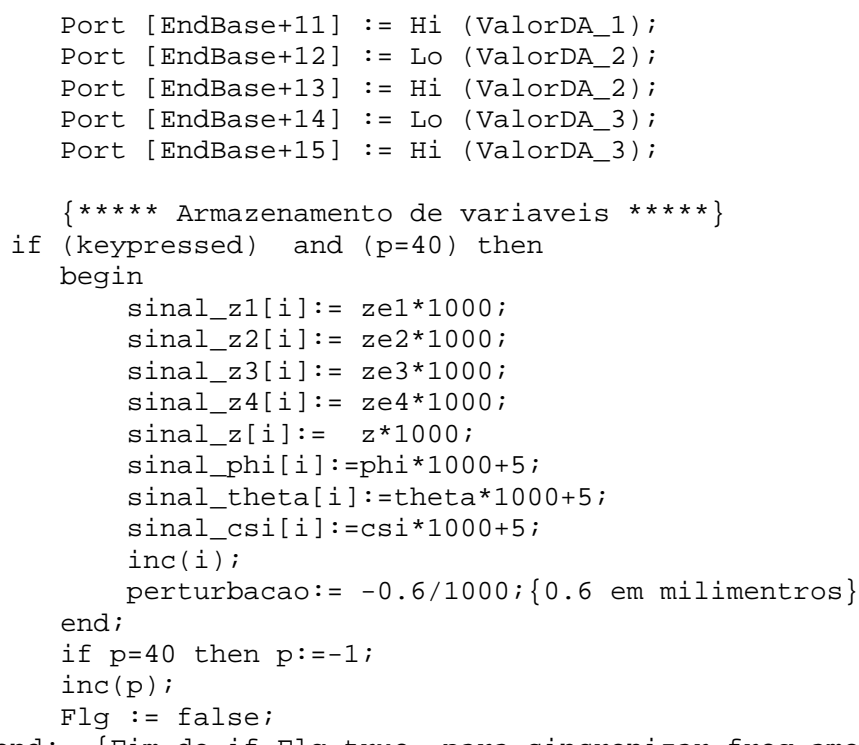

end; \{Fim do if $\mathrm{Flg}=$ true, para sincronizar freq amostragem\} until $(i=501)$ \{keypressed\} or (fIntoverRun) or $($ nAms $=10000)$;

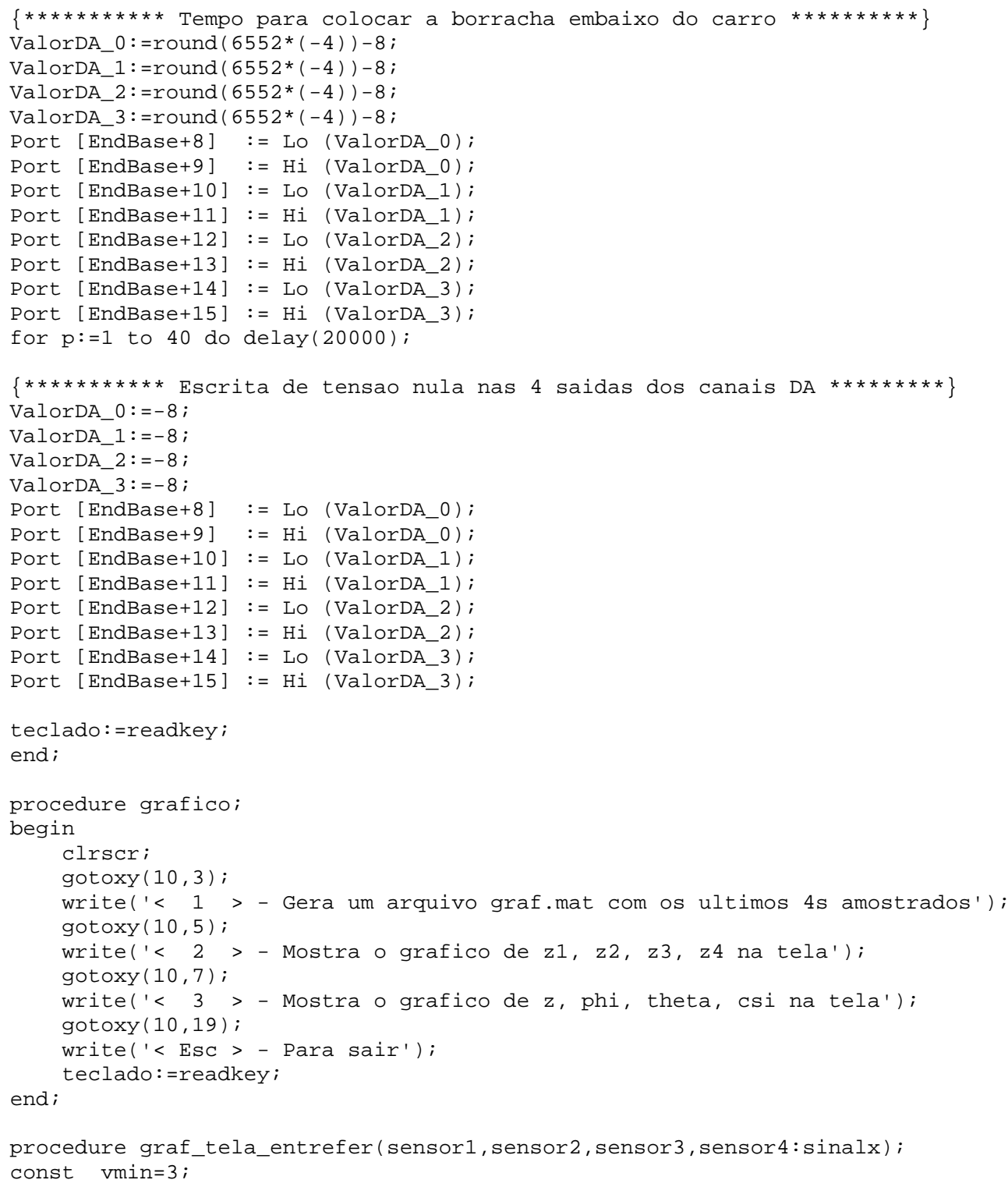




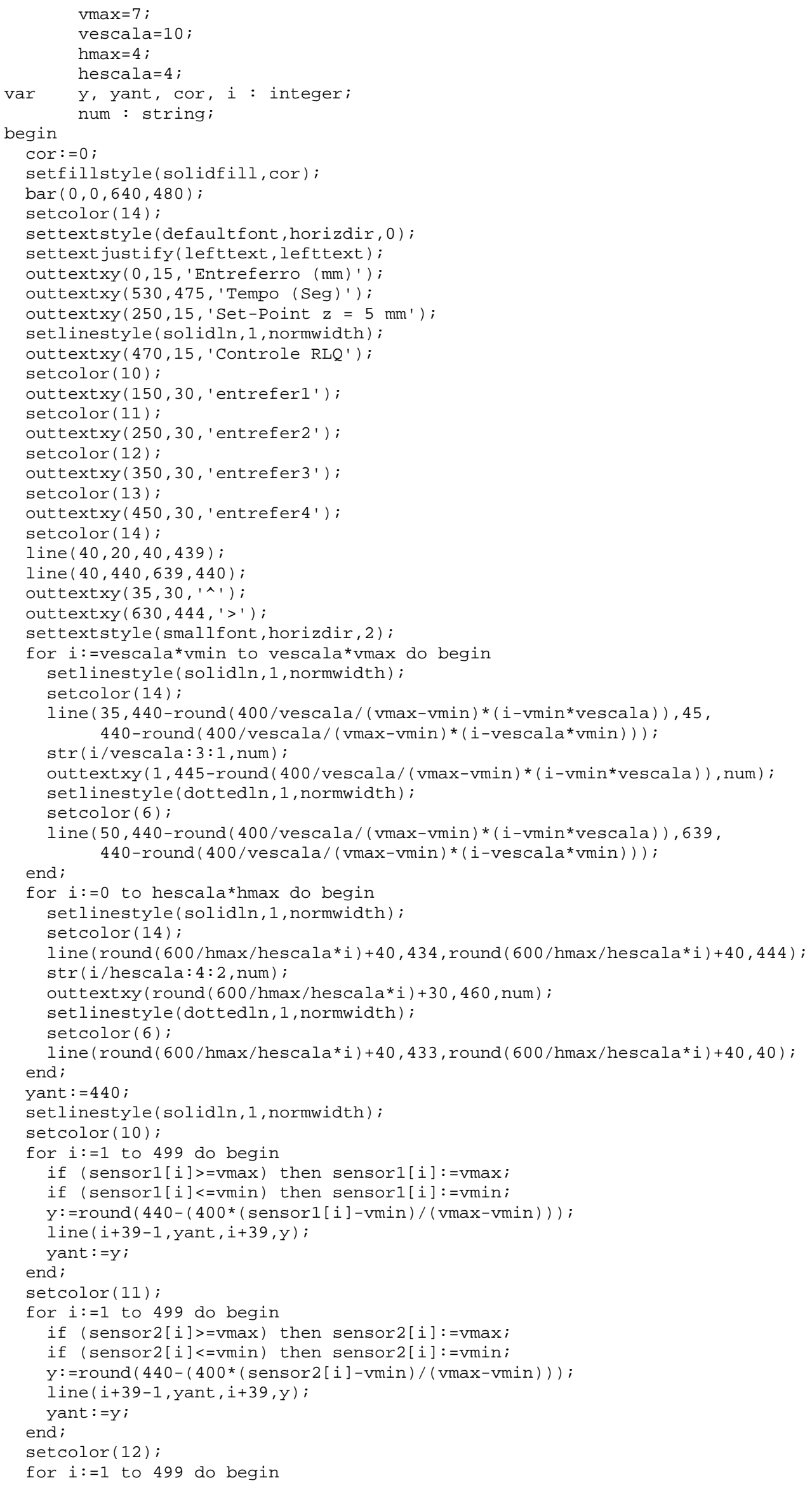




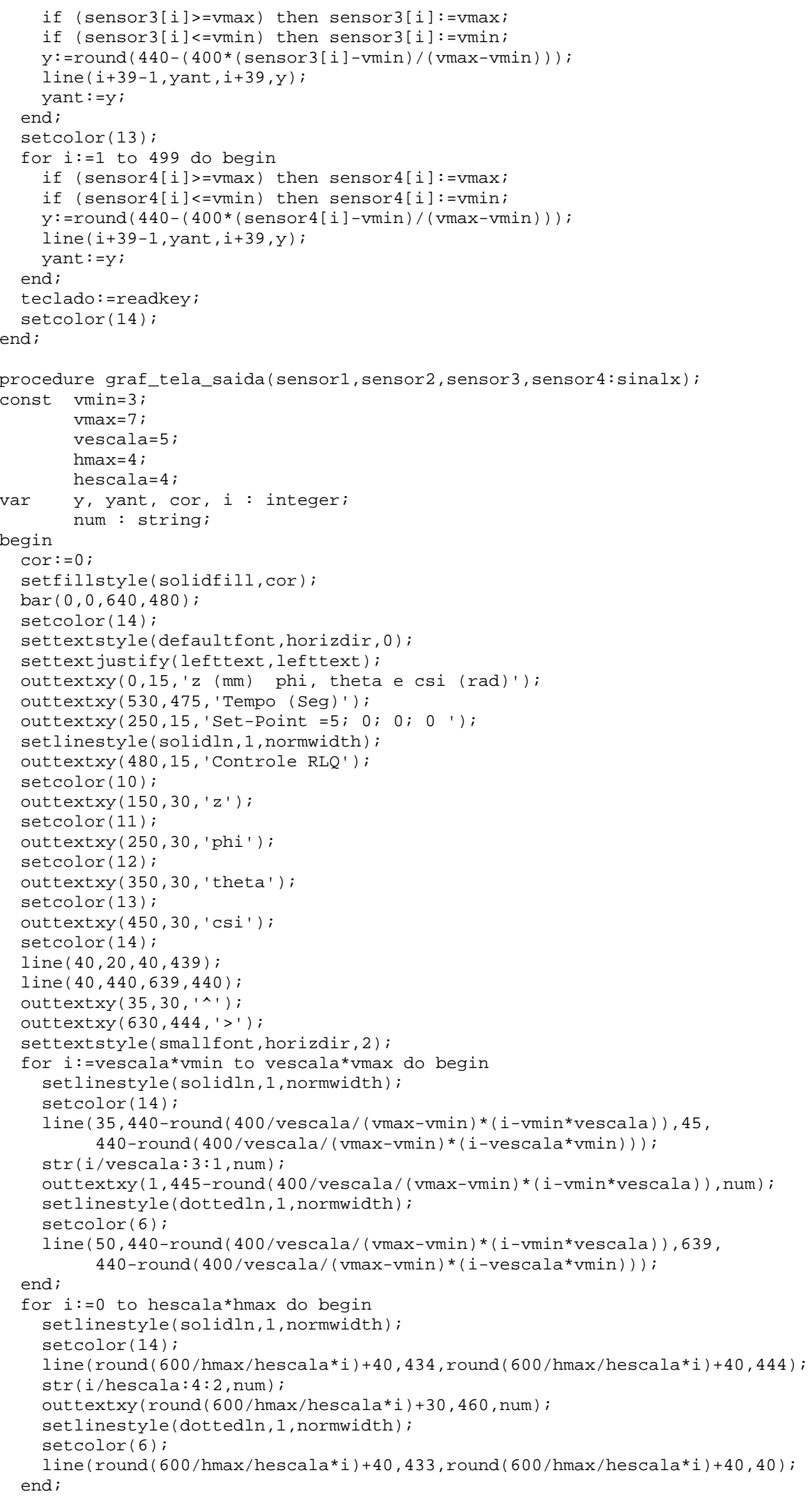




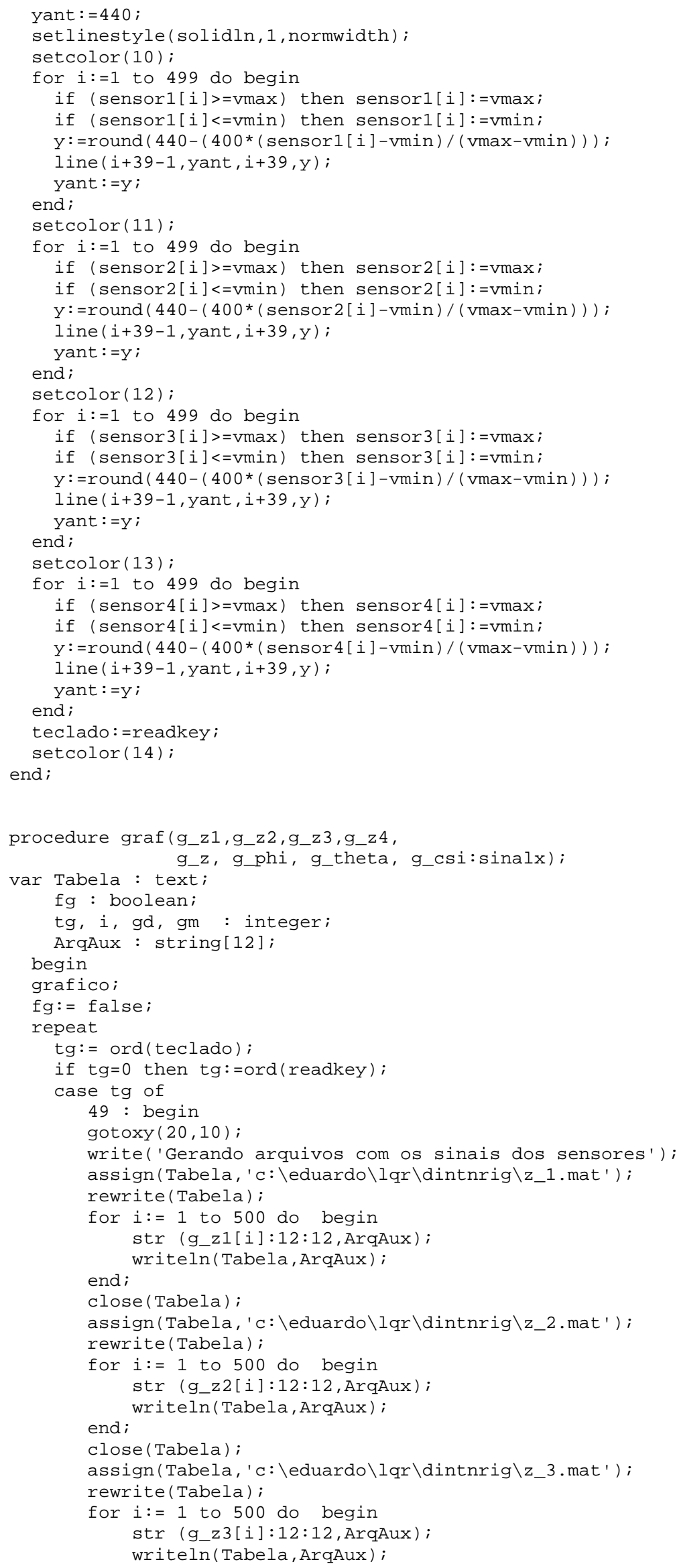




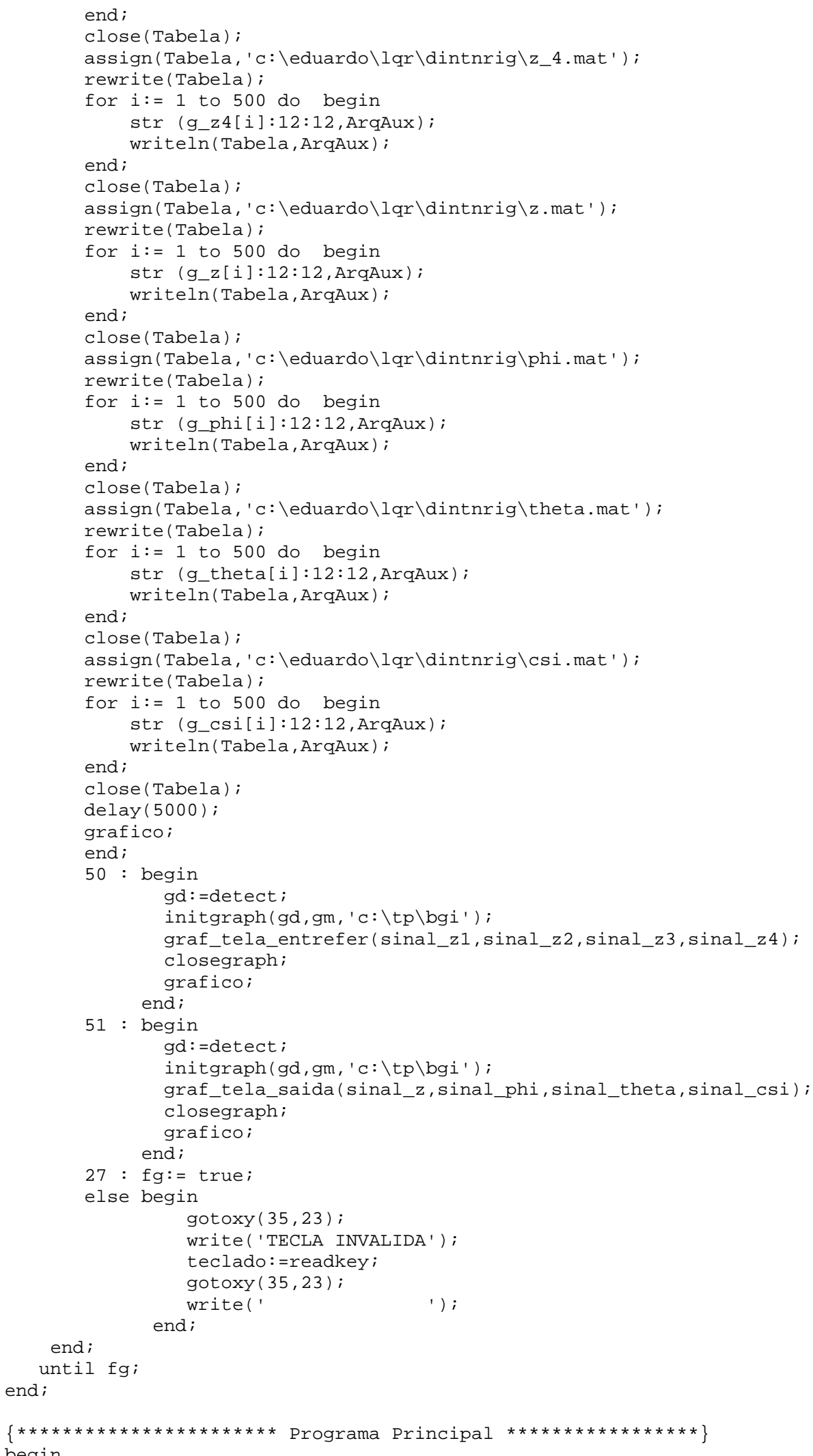




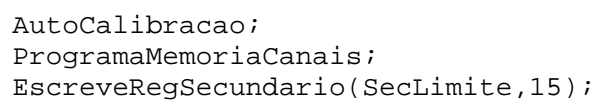

\{ Comando de Auto-Calibracao do Conversor A/D \{ Programa a memoria de canais

\{Carrega numero de canais menos 1 no Reg.Limite\}

\{ver obs. procedimento trataint para num canais\}

\{ Zera Ponteiro da memoria de canais

$\{$ Limpa a memoria FIF0 da CAD12/36

\{ Inicia vetor de interrupcao

$\left\{\right.$ observe que o vetor usado $\mathrm{e}^{\prime}$ IntUsada +8 \}

\{ limpa FF de pedido de interrupcao

\{ flag para indicar ocorrencia de interrupcao $\}$

\{Procedimento de AutoCalibAceler;

assign(referen, 'c: \eduardo \lqr \projetos \referen.bin' );

reset (referen);

for $i:=1$ to 4 do $\operatorname{read}(r e f e r e n, \operatorname{refer}[i])$;

close(referen);

delay (5000);

ref_yA:=refer[1]; ref_yB:=refer[2]；ref_yc:=refer[3]；ref_yD:=refer[4];

FacaAlgo；

\{ faz algo junto com as interrupcoes \}

graf(sinal_z1,sinal_z2, sinal_z3,sinal_z4,

clrscr;

SetIntVec (IntUsada+8, SvVetInt);

InibeInt;

$\{$ inibe a interrupcao 


\section{REFERÊNCIAS BIBLIOGRÁFICAS}

1. ANALOG DEVICES. ADXL 105EB Accelerometer evaluation board, 1999a.

2. ANALOG DEVICES. ADXL 105 High Accuracy $+/-1$ g to $+/-5$ g Single Axis iMEMS Acclerometer with Analog Input, 1999b.

3. ATHANS, M. 6.232-Multivariable Control System. The behavior of the closed-loop poles using the LQR design methodology: the root-square locus for siso systems. Lecture Notes, MIT, 1981.

4. BITTAR, A. Levitação magnética de uma barra flexível. 1993. 158p. Dissertação (Mestrado) - Escola Politécnica, Universidade de São Paulo. São Paulo, 1993.

5. BITTAR, A. Controle da suspensão eletromagnética do protótipo de um veículo. 1998. 219p. Tese (Doutorado) - Escola Politécnica, Universidade de São Paulo. São Paulo, 1998.

6. CAMPO, A. B. Controle de propulsão e levitação de um MAGLEV eletrodinâmico. 2001. 131p. Tese (Doutorado) - Escola Politécnica, Universidade de São Paulo. São Paulo, 2001.

7. CASSAT, A.; JUGER, M. MAGLEV Projects Technology Aspects and Choices. IEEE Transactions on Applied Superconductivity, v.12, n.1, p. 915-925, March, 2002.

8. COSTA, E. A. et al. Control and optimization of the electromagnetic suspension operation of a Maglev vehicle. $17^{\text {th }}$ International Congress of Mechanical Engineering. São Paulo, November 2003. paper 1020. 1 CDROM.

9. CRUZ, J. J. Controle robusto multivariável. São Paulo: Edusp, 1996. 
10. EARNSHAW, S. On the nature of the molecular forces which regulate the constitution of the luminferous ether. Trans. Cambridge Philosophical Soc., v.7, p.97-112, 1842 .

11. FALCONE, A.G. Eletromecânica. São Paulo: Edgar Blucher, 1985.

12. GAVIN, H. P.; MORALES, R.; REILLY, K. Drift-free Integrators. Review of scientific Instruments, v.69, n.5, p.2171-2175, May 1998.

13. GAVIN, H. P. Control of seismically-excited vibration using electrorheological materials and Lyapunov methods. IEE Transactions on Automation Control, v.9, n.1, p.27-37, January 2001.

14. KAILATH, T. Linear systems. USA: Prentice Hall Inc., 1980.

15. LEVINE, W.S. (editor) The Control HandBook. USA: CRC Press Inc., 1996.

16. LYNX TECNOLOGIA. CAD 12/36 - Manual do usuário e de referência. São Paulo, 1997.

17. OGATA, K. Engenharia de Controle Moderno. 3.ed. Rio de Janeiro: Prentice Hall do Brasil, 1998.

18. ORSINI, L.Q. Introdução aos sistemas dinâmicos. Rio de Janeiro: Guanabara Dois, 1985.

19. ORSINI, L.Q. Eletromagnetismo. São Paulo: EPUSP, 1984.

20. POST, R.F.; RYUTOV, D.D. The Inductrack: a simpler approach to magnetic levitation. IEEE Transactions on Applied Superconductivity, p.901-904, March, 2000.

21. SINHA, P.K. Electromagnetic suspension: dynamics and control. London: Peter Peregrinus Ltd, 1987.

22. VALADARES, E. A. Ciência Hoje, v. 30, n.178, p.8, Dezembro, 2001.

23. VUCHIC, V.R.; CASELLO, J.M. An evaluation of Maglev technology and its comparison with high speed rail. Trasportation Quarterly, v.56, n.2, p.33-49, 2002. 
24. ZHOU, K; DOYLE, J. C. Robust and optimal control. New Jersey: Prentice Hall, 1996. 